FZJ-IKP-TH-2010-08, HISKP-TH-10/09

\title{
Effect of charmed meson loops on charmonium transitions
}

\author{
Feng-Kun Guo ${ }^{1 *}$ C. Hanhart ${ }^{1,2 \dagger}$, Gang Li ${ }^{3,4 \ddagger}$, Ulf-G. Meißner ${ }^{1,2,5 \S}$, and Qiang Zhao ${ }^{3,6}$ \\ ${ }^{1}$ Institut für Kernphysik and Jülich Center for Hadron Physics, \\ Forschungszentrum Jülich, D-52425 Jülich, Germany \\ ${ }^{2}$ Institute for Advanced Simulation, Forschungszentrum Jülich, D-52425 Jülich, Germany \\ ${ }^{3}$ Institute of High Energy Physics, Chinese Academy of Sciences, Beijing 100049, China \\ ${ }^{4}$ Department of Physics, Qufu Normal University, Qufu, 273165, China \\ ${ }^{5}$ Helmholtz-Institut für Strahlen- und Kernphysik and Bethe Center for Theoretical Physics, \\ Universität Bonn, D-53115 Bonn, Germany \\ ${ }^{6}$ Theoretical Physics Center for Science Facilities, CAS, Beijing 100049, China
}

January 20, 2011

\begin{abstract}
The effects of intermediate charmed mesons on charmonium transitions with the emission of one pion or eta are studied systematically. Based on a non-relativistic effective field theory we show that charmed meson loops are enhanced compared to the corresponding tree-level contributions for transitions between two $S$-wave charmonia as well as for transitions between two $P$-wave charmonia. On the contrary, for the transitions between one $S$-wave and one $P$-wave charmonium state, the loops need to be analyzed case by case and often appear to be suppressed. The relation to and possible implications for an effective Lagrangian approach are also discussed. This study at the same time provides a cross check for the numerical evaluations.
\end{abstract}

PACS: $13.25 . \mathrm{Gv}, 14.40 . \mathrm{Pq}, 12.39 . \mathrm{Fe}$

\footnotetext{
*E-mail address: f.k.guo@fz-juelich.de. Current address: Helmholtz-Institut für Strahlen- und Kernphysik, Universität Bonn, D-53115 Bonn, Germany

${ }^{\dagger}$ E-mail address: c.hanhart@fz-juelich.de

${ }^{\ddagger}$ E-mail address: gli@ihep.ac.cn

$\S$ E-mail address: meissner@hiskp.uni-bonn.de

ฯ E-mail address: zhaoq@ihep.ac.cn
} 


\section{Introduction}

Since the discovery of the $J / \psi$ more than thirty years ago, the decays of heavy quarkonia have played an important role in the physics of quarks and hadrons. During the past decades, experimental studies of the heavy quarkonia at CLEO, Belle, BaBar, CDF, D0, and BESII have provided great opportunities for examining many interesting properties of Quantum Chromodynamics (QCD). At the present stage, BES-III [1] has accumulated the largest data samples for $J / \psi$ and $\psi^{\prime}$ decays, and $\overline{\mathrm{P}} \mathrm{ANDA}[2]$ plans to accumulate data for charmonia which cannot be produced directly in electron-positron annihilations. These facilities will deepen our understanding of the charmonium physics, and hence the non-perturbative aspects of QCD. Although many theoretical investigations have been performed in the past thirty years (for comprehensive reviews, see Refs. 3, 1, 4]), there remain many mysteries in charmonium physics to be settled. On the contrary, due to the new experimental data with unprecedented statistics, many new interesting problems have appeared, e.g. the nature of many of the new $X, Y, Z$ resonances discovered in the charmonium mass region has still not been well understood (for recent reviews, see, e.g. Refs. [5, 6, 7, 8, 9]).

Furthermore, various recent phenomenological calculations suggest that charmed meson loops may play an important role in the decays of heavy quarkonia (for an overview, see [10]). For instance, using an effective Lagrangian approach (ELA), intermediate heavy-meson loop contributions are found to be essential for understanding the puzzling $\psi(3770)$ non- $D \bar{D}$ decays [11, 12. They are also important in the $J / \psi$ decays into a vector and a pseudoscalar meson [13] and in the $M 1$ radiative transitions between two charmonia [14. Besides, using the on-shell approximation, the bottom meson loops were suggested to make the $\Upsilon(5 S)$ transitions to the lower $\Upsilon$ states with the emission of two pions [15] or one $\eta$ [16] different from those of the $\Upsilon(4 S)$. The inclusion of intermediate heavy mesons in heavy quarkonium transitions, sometimes called coupled-channel effects, has been noticed for more than twenty years [17, 18, 19. Also, the effect of the mass differences between the neutral and charged mesons in the intermediate states (i.e. in the meson loops) plays a role in other isospin breaking processes. This effect, known to be of particular importance near the continuum thresholds, was already studied in the $\eta^{\prime}$ decays [20], and in the decays $\phi \rightarrow \omega \pi^{0}$ [21, 22], $J / \psi \rightarrow \phi \eta \pi^{0}$ [23, 24], and $D_{s 0}(2317) \rightarrow D_{s} \pi^{0}[25,26,27$.

An often used formalism dealing with the hadronic transitions between two heavy quarkonia is the QCD multipole expansion (QCDME) [28, 29, 30. The QCDME is based on the assumption that the emitted gluons are soft so that their wavelengths are much larger than the size of a heavy quarkonium. As a result, a multipole expansion similar to that in classical electrodynamics can be performed. The soft gluons then hadronize into light meson(s), for instance the pion(s) or eta, and the matrix elements may be worked out using soft pion theorems. A schematic diagram for the multipole transition from a heavy quarkonium to another one with the emission of one pion is plotted in Fig. 1(a). However, this ansatz clearly misses the contribution from intermediate mesons. This can be understood as a heavy quarkonium can couple to a heavy meson and heavy anti-meson pair through the non-perturbative production of a light quark and anti-quark pair, see Fig. 1(b). In Refs. [19, 31, within the framework of QCDME, the intermediate heavy meson effects were considered to account for non-multipole effects in the di-pion transitions between two charmonia, the $\psi$ states, or bottomonia, the $\Upsilon$ states. Better agreement with the experimental data was obtained.

In light of these phenomenological indications, it is important to have a theoretical formalism which has a controlled uncertainty to study the effects of heavy meson loops in the 


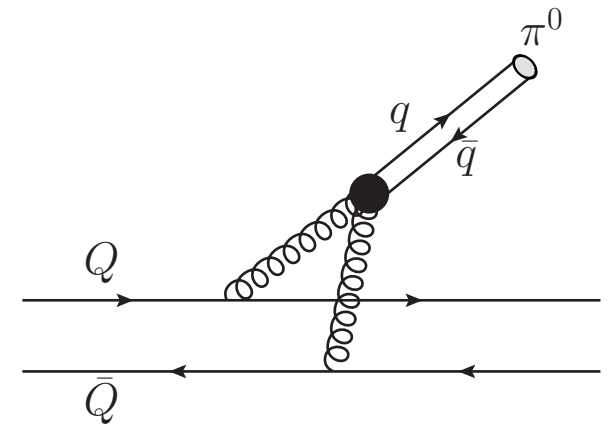

(a)

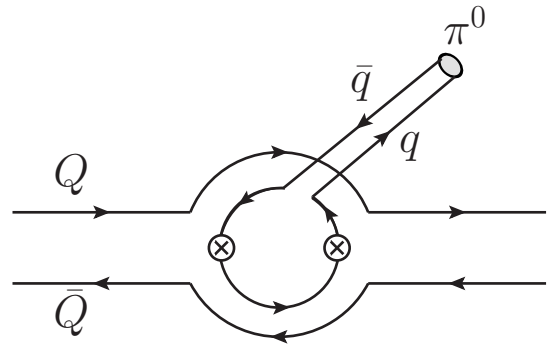

(b)

Figure 1: Schematic diagrams of the QCDME mechanism (a) and the non-multipole (b) effects of the intermediate heavy meson loops for heavy quarkonium transition with the emission of one pion.

transitions of heavy quarkonia. In Ref. [32, such a non-relativistic effective field theory (NREFT) formalism was constructed to investigate the charmed meson loops in the decays $\psi^{\prime} \rightarrow J / \psi \pi^{0}(\eta)$. Because the $\psi^{\prime}$ and $J / \psi$ are isospin and $\mathrm{SU}(3)$ flavor singlets, the decay process $\psi^{\prime} \rightarrow J / \psi \pi^{0}$ violates isospin symmetry and $\psi^{\prime} \rightarrow J / \psi \eta$ violates $\mathrm{SU}(3)$ symmetry. Isospin symmetry can be violated by both electromagnetic (e.m.) effects and the mass difference between the $u$ and $d$ quarks. Because the e.m. effects are small [33, 34] (it can be easily shown in the framework of chiral perturbation theory (CHPT) with virtual photons [35, 36], see Section 2.2), these two decays were used to extract the light quark mass ratio $m_{u} / m_{d}[37,38,39,40,41$. The relation between the ratio of the decay widths

$$
R_{\pi^{0} / \eta} \equiv \frac{\mathcal{B}\left(\psi^{\prime} \rightarrow J / \psi \pi^{0}\right)}{\mathcal{B}\left(\psi^{\prime} \rightarrow J / \psi \eta\right)}
$$

and the light quark masses is given by [38, 7]

$$
R_{\pi^{0} / \eta}=3\left(\frac{m_{d}-m_{u}}{m_{d}+m_{u}}\right)^{2} \frac{F_{\pi}^{2}}{F_{\eta}^{2}} \frac{M_{\pi}^{4}}{M_{\eta}^{4}}\left|\frac{\vec{q}_{\pi}}{\vec{q}_{\eta}}\right|^{3},
$$

where $F_{\pi(\eta)}$ and $M_{\pi(\eta)}$ are the decay constant and mass of the pion (eta), respectively. The extracted quark mass ratio using Eq. (1) and the recent measurements of the decay widths from the CLEO Collaboration [42], the BES Collaboration [43] and the Particle Data Group (PDG) fit [4] are listed in Table 1, Comparing with the result obtained using the Goldstone boson masses from leading order (LO) CHPT [45, 46]

$$
\frac{m_{u}}{m_{d}}=\frac{M_{K^{+}}^{2}-M_{K^{0}}^{2}+2 M_{\pi^{0}}^{2}-M_{\pi^{+}}^{2}}{M_{K^{0}}^{2}-M_{K^{+}}^{2}+M_{\pi^{+}}^{2}}=0.56,
$$

the discrepancy is striking. We remark that there might be sizable higher order corrections to this LO result. The up-to-date knowledge of the light quark mass ratio from various determinations including lattice calculations (but excluding $\psi^{\prime}$ decays) was summarized by Leutwyler as $m_{u} / m_{d}=0.47 \pm 0.08$ [47]. The relatively large uncertainty given here thus 


\begin{tabular}{|l|ll|}
\hline \hline & $R_{\pi^{0} / \eta}$ & $m_{u} / m_{d}$ \\
\hline CLEO [42] & $(3.88 \pm 0.23 \pm 0.05) \%$ & $0.40 \pm 0.01$ \\
BES [43] & $(4.8 \pm 0.5) \%$ & $0.35 \pm 0.02$ \\
PDG fit [44] & $(4.0 \pm 0.3) \%$ & $0.39 \pm 0.02$ \\
\hline \hline
\end{tabular}

Table 1: The light quark mass ratio $m_{u} / m_{d}$ extracted using Eq. (1) from the recent experimental measurements by different collaborations.

provides an overlap with the results quoted in Table 1, however, only at the very low end. In Ref. [32] it was stressed that for the mentioned transitions the effects of charmed meson loops, ignored in the previous analyses, should be sizable. The charmonia $\psi^{\prime}$ and $J / \psi$ couple to charmed and anti-charmed mesons, and the pion is emitted from one intermediate charmed meson. The proper expansion parameter is the velocity of the intermediate meson, which for below threshold decays is defined via the analytic continuation of the standard definition, namely $v=\sqrt{-E / M_{D}}$, with $E$ measured relative to the open charm threshold. We find $v \approx 0.5$ for most of the decays studied. It is found that loops are enhanced by a factor of $1 / v$ compared to the tree-level contribution where the pion is emitted directly from the charmonium. Therefore the dominant (LO) contributions to these decays come from the loops instead of from the tree graphs which are proportional to the quark mass differences directly, and hence the extraction of quark mass differences from $\psi^{\prime}$ decays mentioned above is not reliable. Stated differently: quarkonium decays could only be used to extract the light quark mass ratio, if either the loop contributions could be controlled quantitatively (at present their uncertainty is quite sizable - see discussion below), or if loop contributions are suppressed. It turned out that the enhancement of the loops in case of the $\psi^{\prime}$ to $J / \psi$ transitions emerges only because the transitions at hand violate isospin or $\mathrm{SU}(3)$ symmetry. The power counting is discussed in detail in Sec. 3.4.

In Ref. [48, the same NREFT is applied to the decays $\psi^{\prime} \rightarrow h_{c} \pi^{0}$ and $\eta_{c}^{\prime} \rightarrow \chi_{c 0} \pi^{0}$. As a consequence of the quantum numbers of the charmonia involved, in these two decays the loop contributions are highly suppressed, and hence the tree-level terms, i.e. the quark mass difference terms, dominate the decay amplitudes. Unfortunately there is no charmonium transition, where one can exploit this observation in order to extract the light quark mass ratio, since typically the phase space available is insufficient for an $\eta$ in the final state. However, in the bottomonium system analogous transitions appear to exist [49], and will allow for the mentioned analysis. This illustrates that the effective field theory at hand predicts a highly non-trivial pattern for the loop contributions in different decays that can be tested experimentally.

In this paper, we will systematically investigate the charmed meson loop contributions to the transitions between two charmonia with the emission of one light pseudoscalar meson. We restrict the charmonia to $S$ and $P$-wave states with radial quantum number $n$ less than or equal to 2 . In other words, we will consider the transitions between or within the following charmonia spin multiplets: $\left\{J / \psi, \eta_{c}\right\},\left\{\chi_{c 0}, \chi_{c 1}, \chi_{c 2}, h_{c}\right\}$ with $n=1$, and $\left\{\psi^{\prime}, \eta_{c}^{\prime}\right\}$, $\left\{\chi_{c 0}^{\prime}, \chi_{c 1}^{\prime}, \chi_{c 2}^{\prime}, h_{c}^{\prime}\right\}$ with $n=2$. Charge conjugation allows for the emission of one light pseudoscalar meson between two charmonia with the same value of $C$. Considering further the constraints from parity conservation, all the allowed transitions are plotted in Fig. 2, 11 and

\footnotetext{
${ }^{1}$ In the figure, the masses of the so far unobserved (or unidentified) $2 P$ charmonia are taken from a quark
} 
the following will be considered in the paper:

1) Transitions between two $S$-wave charmonia: $\psi^{\prime} \rightarrow J / \psi \pi^{0}$, and $\psi^{\prime} \rightarrow J / \psi \eta$.

2) Transitions between one $S$-wave and one $P$-wave charmonium: $\psi^{\prime} \rightarrow h_{c} \pi^{0}, h_{c} \rightarrow J / \psi \pi^{0}$, $h_{c}^{\prime} \rightarrow \psi^{\prime} \pi^{0}, \eta_{c}^{\prime} \rightarrow \chi_{c 0} \pi^{0}, \chi_{c 0} \rightarrow \eta_{c} \pi^{0}$, and $\chi_{c 0}^{\prime} \rightarrow \eta_{c}^{\prime} \pi^{0}$.

3) Transitions between two $P$-wave charmonia: $\chi_{c 0}^{\prime} \rightarrow \chi_{c 1} \pi^{0}, \chi_{c 1}^{\prime} \rightarrow \chi_{c J} \pi^{0}(J=0,1,2)$, $\chi_{c 2}^{\prime} \rightarrow \chi_{c 1(2)} \pi^{0}$, and $h_{c}^{\prime} \rightarrow h_{c} \pi^{0}$.

In fact, as plotted in Fig. 2, the decays $h_{c}^{\prime} \rightarrow J / \psi \pi^{0}(\eta)$ and $\chi_{c 0}^{\prime} \rightarrow \eta_{c} \pi^{0}(\eta)$ can also occur. However, the mass difference between the initial and final charmonium exceeds $800 \mathrm{MeV}$, of order $\mathcal{O}\left(\Lambda_{\chi}\right)$, with $\Lambda_{\chi} \approx 1 \mathrm{GeV}$ denoting the typical hadronic scale. Since the chiral expansion is an expansion in $p / \Lambda_{\chi}$, with $p$ denoting a typical momentum or mass, for those energies the chiral expansion is not expected to converge any more. We therefore do not consider these transitions. There could also be $D$-wave transitions $\chi_{c 2} \rightarrow \eta_{c} \pi^{0}(\eta)$ and $\chi_{c 2}^{\prime} \rightarrow \eta_{c}^{\prime} \pi^{0}$ (not shown in the figure). However, their partial decay widths would be too small to be detected in the near future because of the $D$-wave suppression, the isospin or $\mathrm{SU}(3)$ breaking, and small phase space. They will also not be considered here.

The paper is organized as follows. In Section 2, the tree-level chiral effective Lagrangians and the resulting amplitudes are given for all the decays discussed in the paper. The isospin and $\mathrm{SU}(3)$ breaking are given by quark mass differences. Consistent with earlier analyses, e.m. contributions are found to be small and can be neglected. Various aspects of the charmed meson loops will be discussed in Section 3. Section 4 is devoted to the results of the meson loops for various transitions in the NREFT. In Section 5, a detailed parallel study of the meson loop transitions in the framework of the ELA is presented and the results are compared to those from the NREFT. A brief summary is given in the last section. Various technicalities such as the utilized loop functions, the decay amplitudes from the meson loops and the ingredients of the ELA are given in the Appendices.

\section{Effective Lagrangians for tree-level diagrams}

Isospin breaking has two sources. One is the mass difference between the up and down quarks, and the other one is of e.m. origin because photons do not have definite isospin. This section is devoted to the construction of the LO chiral Lagrangians for the tree-level diagrams of the transitions considered in this paper. Both the quark mass difference and the e.m. effects will be taken into account.

The chiral effective Lagrangians are of the most general form which is invariant under the transformations of $\mathrm{SU}(3)_{L} \times \mathrm{SU}(3)_{R}$, parity and charge conjugation. The charmonia are treated as matter fields, and the pion and eta are the Goldstone bosons of the spontaneous breaking of $\mathrm{SU}(3)_{L} \times \mathrm{SU}(3)_{R}$ down to its vector subgroup $\mathrm{SU}(3)_{V}$. The charmonia are $\mathrm{SU}(3)$ singlets, so they do not change under the chiral transformation. The Goldstone boson fields

$$
\phi=\left(\begin{array}{ccc}
\frac{1}{\sqrt{2}} \pi^{0}+\frac{1}{\sqrt{6}} \eta & \pi^{+} & K^{+} \\
\pi^{-} & -\frac{1}{\sqrt{2}} \pi^{0}+\frac{1}{\sqrt{6}} \eta & K^{0} \\
K^{-} & \bar{K}^{0} & -\frac{2}{\sqrt{6}} \eta
\end{array}\right),
$$

model calculation considering the color-screening effect due to the light quark and anti-quark pair creation [50]. Note that these values are only used for illustration. 


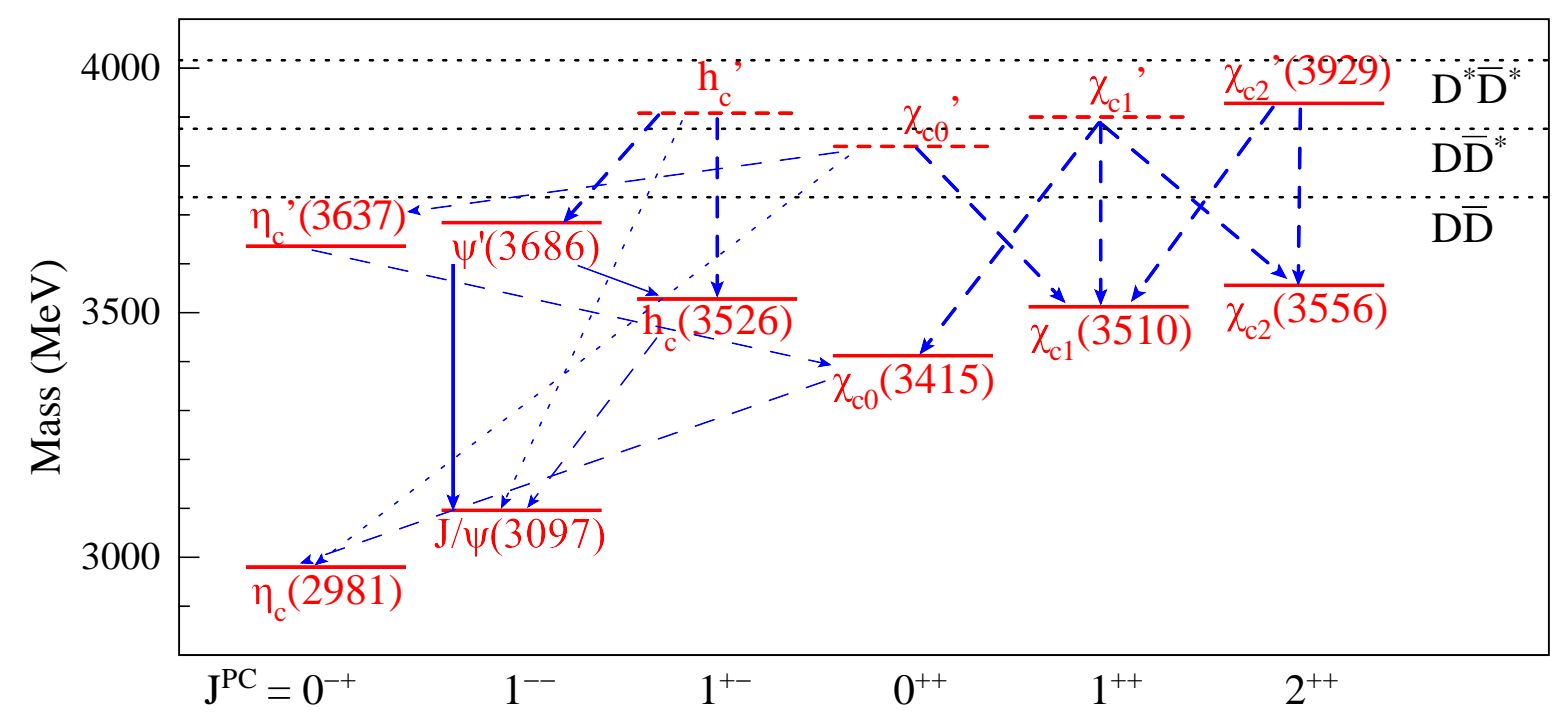

Figure 2: All possible $S$ and $P$-wave transitions among the ground state and the first radial excited $S$ and $P$-wave charmonia with the emission of one light pseudoscalar meson $\left(\pi^{0}, \eta\right)$. The unobserved resonances and decay modes are plotted in dashed. The masses for the unobserved resonances are taken from predictions in Ref. [50]. The thresholds for the $D \bar{D}$, $D \bar{D}^{*}$ and $D^{*} \bar{D}^{*}$ are represented by the dotted horizontal lines. The solid lines represent the measured decays. The dashed and dotted lines are not yet measured with only the former discussed in the paper and the latter are beyond the range of the applicability of the chiral EFT (but can be considered in the model-dependent ELA also discussed here). Thick lines indicate transitions with enhanced charmed meson loops.

where we have approximated the $\eta$ as one element of the octet $\mathrm{SU}(3)$ representation, are collected in $u=\exp (i \phi / \sqrt{2} F)$ with $F$ being the pion decay constant in the chiral limit. Under the transformation of $\mathrm{SU}(3)_{L} \times \mathrm{SU}(3)_{R}$, we have

$$
u \rightarrow R u h^{\dagger}=h u L^{\dagger},
$$

where $h$ is the compensator field. It is convenient to construct chiral Lagrangians using operators whose chiral transformation is $O \rightarrow h O h^{\dagger}$. The following such building blocks will be used

$$
\begin{aligned}
u_{\mu} & =i\left(u^{\dagger} \partial_{\mu} u+\partial_{\mu} u u^{\dagger}\right) \\
\chi_{ \pm} & =u^{\dagger} \chi u^{\dagger} \pm u \chi^{\dagger} u \\
Q_{ \pm} & =\frac{1}{2}\left(u^{\dagger} Q u \pm u Q u^{\dagger}\right)
\end{aligned}
$$

where the diagonal quark mass matrix and the charge matrix are

$$
\begin{aligned}
\chi & =2 B_{0} \cdot \operatorname{diag}\left(m_{u}, m_{d}, m_{s}\right), \\
Q & =e \cdot \operatorname{diag}(2 / 3,-1 / 3,-1 / 3),
\end{aligned}
$$

in terms of $B_{0}=|\langle 0|\bar{q} q| 0\rangle| / F^{2}$ and the elementary electric charge $e(e>0)$. 
In the heavy quark limit with $m_{Q} \rightarrow \infty$, the coupling of a heavy quark to a gluon is spin-independent [51]. As a result, there is a spin symmetry in that limit, and the heavy quarkonia, which differ from each other only in the total spin of the heavy quark and antiquark, can be grouped into the same spin multiplet. It is then convenient to introduce a single field for a spin multiplet of heavy quarkonia [52, 53] using the trace formalism proposed for single-heavy mesons [54, 55]. In this way, the consequence of the heavy quark spin symmetry can be obtained automatically by evaluating a trace in spinor space. The construction of charmonium fields with arbitrary orbital angular momentum $l$ in the trace formalism can be found in Ref. [53. Since we are dealing with the transitions between two charmonia, the heavy quark four-velocity is conserved up to higher order corrections. In this case, it is convenient to use the two-component notation as introduced in Ref. [56]. For doing that, the four-velocity is chosen to be $v^{\mu}=(1, \overrightarrow{0})$. In the two-component notation, the field for the $S$-wave charmonia reads

$$
J=\vec{\psi} \cdot \vec{\sigma}+\eta_{c}
$$

with $\vec{\psi}$ and $\eta_{c}$ annihilating the $J / \psi$ and $\eta_{c}$ states. The field for the $P$-wave charmonia is 2

$$
\chi^{i}=\sigma^{j}\left(-\chi_{c 2}^{i j}-\frac{1}{\sqrt{2}} \epsilon^{i j k} \chi_{c 1}^{k}+\frac{1}{\sqrt{3}} \delta^{i j} \chi_{c 0}\right)+h_{c}^{i}
$$

where $\chi_{c 2}^{i j}, \chi_{c 1}^{i}, \chi_{c 0}$ and $h_{c}$ annihilate the $\chi_{c 2}, \chi_{c 1}, \chi_{c 0}$ and $h_{c}$ states, respectively. $\chi_{c 2}^{i j}$ is a symmetric and traceless tensor.

The quantum numbers of the charmonia determine their parity and charge conjugation transformation properties. The parity transformations for the charmonia fields are given by

$$
J \stackrel{\mathcal{P}}{\rightarrow}-J, \quad \chi^{i} \stackrel{\mathcal{P}}{\rightarrow} \chi^{i},
$$

and the charge conjugation transformations are given by

$$
\begin{aligned}
J \stackrel{\mathcal{C}}{\rightarrow} \sigma_{2} J^{T} \sigma_{2} & =-\vec{\psi} \cdot \vec{\sigma}+\eta_{c}, \\
\chi^{i} \stackrel{\mathcal{C}}{\rightarrow}-\sigma_{2} \chi^{i} \sigma_{2} & =\sigma^{j}\left(-\chi_{c 2}^{i j}-\frac{1}{\sqrt{2}} \epsilon^{i j k} \chi_{c 1}^{k}+\frac{1}{\sqrt{3}} \delta^{i j} \chi_{c 0}\right)-h_{c}^{i},
\end{aligned}
$$

where $J^{T}$ is the transpose of $J$. Denoting the rotation in the $\mathrm{SU}(2)$ spin space of the heavy quark (anti-quark) by $S(\bar{S})$, the transformation of the charmonium fields reads

$$
J \stackrel{\mathcal{S}}{\rightarrow} S J \bar{S}^{\dagger}, \quad \chi^{i} \stackrel{\mathcal{S}}{\rightarrow} S \chi^{i} \bar{S}^{\dagger}
$$

The transformation for a heavy quarkonium field with arbitrary orbital angular momentum is given in 53] in four-component notation. In two-component notation, the transformation properties for the $\chi_{c J}$ fields can be found in Ref. [57].

\subsection{Quark mass difference}

In the transitions considered in this paper, one pion or eta is emitted. Therefore we need to construct the chiral Lagrangian using the external field $\chi_{-}$, which is proportional to the light

\footnotetext{
${ }^{2}$ The sign convention for the $\chi_{c 2}$ and $\chi_{c 1}$ fields are different from those in Ref. [57.
} 
quark mass matrix and contains an odd number of the Goldstone bosons. Under parity and charge conjugation, $\chi_{-}$transforms as

$$
\chi_{-} \stackrel{\mathcal{P}}{\rightarrow}-\chi_{-}=\chi_{-}^{\dagger}, \quad \chi_{-} \stackrel{\mathcal{C}}{\rightarrow} \chi_{-}^{T},
$$

respectively.

The LO Lagrangian for the transitions between two $S$-wave charmonia can be constructed considering parity conservation, which requires the presence of a derivative, charge conjugation, chiral symmetry and Galilean invariance,

$$
\mathcal{L}_{S S}=\frac{A}{4}\left[\left\langle J^{\prime} \sigma^{i} J^{\dagger}\right\rangle-\left\langle J^{\dagger} \sigma^{i} J^{\prime}\right\rangle\right] \partial^{i}\left(\chi_{-}\right)_{a a},
$$

where $\langle\ldots\rangle$ denotes the trace in spinor space, the subscript $a=u, d, s$ is a flavor index, and $a a$ as a sum over it denotes the trace in flavor space. Similarly, the Lagrangian for the transitions between one $S$ - and one $P$-wave charmonium states is

$$
\mathcal{L}_{S P}=\frac{i}{4} C\left[\left\langle\vec{\chi}^{\dagger} \cdot \vec{\sigma} J^{\prime}\right\rangle+\left\langle J^{\prime} \vec{\sigma} \cdot \vec{\chi}^{\dagger}\right\rangle\right]\left(\chi_{-}\right)_{a a},
$$

and that for the transitions between two $P$-wave charmonia is

$$
\mathcal{L}_{P P}=i \frac{\gamma}{2} \epsilon^{i j k}\left\langle\chi^{i \prime} \chi^{j \dagger}\right\rangle \partial^{k}\left(\chi_{-}\right)_{a a},
$$

These Lagrangians were first proposed in Ref. [58] in four-component notation. Note that due to the presence of a Pauli matrix between the two heavy quarkonium fields in Eqs. (1314), the heavy quark spin symmetry is violated. On the contrary, the Lagrangian $\mathcal{L}_{P P}$ preserves the spin symmetry.

The goal of the present work is to set up an effective field theory that allows one to systematically study both loop as well as tree level transition amplitudes. To prepare for this we need to assign an order of magnitude estimate to the coupling constants given above. They may be determined in principle as the result of some matching procedure between the hadronic matrix elements and the more fundamental quark-gluon dynamics calculated within (potential) nonrelativistic QCD ((p)NRQCD) — for recent reviews, see Ref. [59, 3, 1, 4]. For instance, the coupling constant $A$ in the Lagrangian Eq. (13) has a mass dimension -2 . There are several different scales in the physics related to heavy quarkonia. They are the heavy quark mass $m_{Q}$, the momentum $m_{Q} v_{Q}$, the inverse of which sets the length scale of a heavy quarkonium, and the energy scale $m_{Q} v_{Q}^{2}\left[60\right.$ ], where $v_{Q}$ denotes the velocity of the heavy quark within a heavy quarkonium to be distinguished from the velocity $v$ of the heavy mesons in the loops to be introduced in the next section - for an estimate of the values of various scales in heavy quarkonia, one may refer to [61]. In addition, there is the nonperturbative QCD scale $\Lambda_{\mathrm{QCD}}$. In this paper, we are considering the low-lying heavy quarkonia. For these states, it is believed that $m_{Q} v_{Q}^{2} \gtrsim \Lambda_{\mathrm{QCD}}$, which defines the weak-coupling regime [59] - e.g. with $v_{c}^{2} \simeq 0.3$ and $m_{c}=1.5 \mathrm{GeV}$ we find $m_{Q} v_{Q}^{2} \simeq 450 \mathrm{MeV}$. Since most of the coupling constants introduced in the Lagrangians are dimensionful, they should have certain scaling properties expressed by the above mentioned scales. The tree-level Lagrangian describes a process with the emission of soft gluons, which then hadronize into a pion or an eta. As mentioned in the Introduction, the applicable regime of our effective field theory is limited to the transitions with the pion (eta) energy much smaller than $\Lambda_{\chi}$, i.e. $E_{\pi(\eta)} \lesssim 600 \mathrm{MeV}$. 
Hence the energy of the emitted gluons should also be $\lesssim 600 \mathrm{MeV}$. Therefore, the proper dimensionful parameter that sets the scale for this nonperturbative process should be either $m_{c} v_{c}^{2}$, which is sometimes called ultrasoft, or $\Lambda_{\mathrm{QCD}}$. As mentioned above, the charmonia considered in this paper are weakly-coupled, i.e. $m_{c} v_{c}^{2} \gtrsim \Lambda_{\mathrm{QCD}}$. So conservatively, one may take $\Lambda_{\mathrm{QCD}}$ to set the soft scale. Furthermore, since the transition violates spin symmetry we have to put in a factor $\Lambda_{\mathrm{QCD}} / m_{c}$ to finally get

$$
A \sim \frac{1}{\Lambda_{\mathrm{QCD}} 2 m_{c}} \frac{\Lambda_{\mathrm{QCD}}}{m_{c}}=\frac{1}{2 m_{c}^{2}}
$$

where a factor of $1 /\left(2 m_{c}\right)$ was introduced to make $A$ have the correct dimension. In addition, assigning the pion decay constant as $F \sim \Lambda_{\mathrm{QCD}}$, and the quark condensate as $|\langle 0|\bar{q} q| 0\rangle| \sim$ $\Lambda_{\mathrm{QCD}}^{3}$, one has

$$
B_{d u}=\frac{B_{0}}{F}\left(m_{d}-m_{u}\right) \sim \delta,
$$

with $\delta$ denoting the quark mass difference. So using the expressions given in Table 2 , the tree-level amplitude for a pionic transition scales as

$$
\mathcal{M}_{\text {tree }}^{S S} \sim \frac{1}{m_{c}} q \delta
$$

where $1 /\left(2 m_{c}\right)$ has been canceled by the factor $\sqrt{M_{i} M_{f}}$ due to nonrelativistic normalization.

The dimension of the coupling constant $\gamma$ in the Lagrangian for the transitions between two $P$-wave quarkonia is the same as that of $A$. However, for these transitions, the spin symmetry is preserved as can be seen from Eq. (15). So in the scaling of $\gamma$ the suppression factor $\Lambda_{\mathrm{QCD}} / m_{c}$ should not be present. This is the only difference from that of $A$. The dimension of $C$ in Eq. (14) is higher than that of $A$ or $\gamma$ by one unit. Therefore, analogous to Eq. (18), the scaling of the tree-level amplitude for a pionic transition between two $P$-wave charmonia and that for a transition between one $S$ - and one $P$-wave states should be given by

$$
\mathcal{M}_{\text {tree }}^{P P} \sim \frac{1}{\Lambda_{\mathrm{QCD}}} q \delta, \quad \mathcal{M}_{\text {tree }}^{S P} \sim \delta
$$

\section{$2.2 \quad$ Virtual photons}

The e.m. effects come from virtual photons exchanged in the processes. The inclusion of virtual photons has been first considered systematically for three-flavor CHPT in Ref. [36].

Since the photons are virtual, we need to consider operators with at least two powers of electric charge. At $\mathcal{O}\left(m_{q}^{0} \alpha\right)$, with $\alpha \equiv e^{2} /(4 \pi) \simeq 1 / 137$ the fine structure constant, one virtual photon is exchanged. There are three types of operators, and we will discuss them one by one:

1) The virtual photon is exchanged between light quarks, which is the standard case in CHPT with virtual photons. One needs quadratic combinations of the spurions $Q_{+}$and $Q_{-}$which act on the light quarks. The parity and charge conjugation properties of the $Q_{ \pm}$are given by 62 ]

$$
Q_{ \pm} \stackrel{\mathcal{P}}{\rightarrow} \pm Q_{ \pm}^{\dagger}, \quad Q_{ \pm} \stackrel{\mathcal{C}}{\rightarrow} \pm Q_{ \pm}^{T}
$$


There is only one light pseudoscalar meson in the final states of all the transitions considered in the paper. $Q_{+}$and $Q_{-}$contain even and odd number of the Goldstone fields, respectively, and their expansion reads

$$
\begin{aligned}
& Q_{+}=Q+\mathcal{O}\left(\phi^{2}\right) \\
& Q_{-}=\frac{i}{\sqrt{2} F}(Q \phi-\phi Q)+\mathcal{O}\left(\phi^{3}\right) .
\end{aligned}
$$

Therefore, at $\mathcal{O}(\alpha)$, the possible virtual photon operators for the one pion (eta) emission transitions between charmonia are $\left(Q_{+} Q_{-}\right)_{a a}$ and $\left(Q_{+}\right)_{a a}\left(Q_{-}\right)_{a a}$. The traces come from the fact that the charmonia are $\mathrm{SU}(3)$ singlets. However, one can easily show that

$$
\begin{aligned}
\left(Q_{-}\right)_{a a} & =0 \\
\left(Q_{+} Q_{-}\right)_{a a} & =0 .
\end{aligned}
$$

Thus, there is no electromagnetic contribution to the one pion emission transitions at order $\mathcal{O}(\alpha)$. Actually, there is a more general relation

$$
\left(Q_{+}^{n} Q_{-}\right)_{a a}=0+\mathcal{O}\left(\phi^{3}\right) .
$$

That means, for any transition with the emission of one soft pion between two iso-singlets, the contribution from virtual photons exchanged between light quarks vanishes at treelevel.

2) The virtual photon is exchanged inside the heavy quarkonia. In this case, no operator containing light mesons without derivative or quark mass can be constructed. This may be understood as virtual photons exchanged inside the heavy quarkonia cannot contribute to the isospin breaking transitions.

3) The virtual photon is exchanged between a heavy (anti-)quark and a light (anti-)quark. This kind of virtual photon is important in understanding the isospin mass splitting of heavy hadrons [63, 64]. In principle, this will give a non-vanishing contribution to isospin breaking transitions. For the transitions considered here, however, only one Goldstone boson is emitted. Therefore, the operator for the light flavor part should be $\left(Q_{-}\right)_{a a}$. However, the trace of $Q_{-}$vanishes (see Eq. (22)).

Therefore, there is no e.m. contribution to the isospin breaking heavy quarkonium transitions at order $\mathcal{O}(\alpha)$ and thus they can be neglected compared to the quark mass difference terms. This conclusion agrees with those of earlier studies in Refs. [33, 34].

\subsection{Tree-level amplitudes}

Before working out the tree-level amplitudes using the Lagrangians given in Section 2.1, one subtlety needs to be addressed. In Eq. (3), the $\pi^{0}$ and $\eta$ are $\mathrm{SU}(3)$ flavor eigen-states. However, they are not exactly the same as the physical pion and eta which are mass eigenstates. Denoting the physical states by $\tilde{\pi}^{0}$ and $\tilde{\eta}$, the $\pi^{0}-\eta$ mixing is given as

$$
\begin{gathered}
\pi^{0}=\tilde{\pi}^{0} \cos \epsilon_{\pi^{0} \eta}-\tilde{\eta} \sin \epsilon_{\pi^{0} \eta}=\tilde{\pi}^{0}-\epsilon_{\pi^{0} \eta} \tilde{\eta}+\mathcal{O}\left(\epsilon_{\pi^{0} \eta}^{2}\right), \\
\eta=\tilde{\eta} \cos \epsilon_{\pi^{0} \eta}+\tilde{\pi}^{0} \sin \epsilon_{\pi^{0} \eta}=\tilde{\eta}+\epsilon_{\pi^{0} \eta} \tilde{\pi}^{0}+\mathcal{O}\left(\epsilon_{\pi^{0} \eta}^{2}\right),
\end{gathered}
$$




\begin{tabular}{|l|l|}
\hline \hline$\psi^{\prime} \rightarrow J / \psi \pi^{0}$ & $i 6 A \epsilon^{i j k} \varepsilon^{i}\left(\psi^{\prime}\right) \varepsilon^{j}(J / \psi) q^{k} B_{d u}$ \\
$\psi^{\prime} \rightarrow J / \psi \eta$ & $i(8 / \sqrt{3}) A \epsilon^{i j k} \varepsilon^{i}\left(\psi^{\prime}\right) \varepsilon^{j}(J / \psi) q^{k} B_{s l}$ \\
$\psi^{\prime} \rightarrow h_{c} \pi^{0}$ & $6 C \vec{\varepsilon}\left(\psi^{\prime}\right) \cdot \vec{\varepsilon}\left(h_{c}\right) B_{d u}$ \\
$\eta_{c}^{\prime} \rightarrow \chi_{c 0} \pi^{0}$ & $6 \sqrt{3} C B_{d u}$ \\
$\chi_{c 0}^{\prime} \rightarrow \chi_{c 1} \pi^{0}$ & $-2 \sqrt{6} i \gamma \vec{\varepsilon}\left(\chi_{c 1}\right) \cdot \vec{q} B_{d u}$ \\
$\chi_{c 1}^{\prime} \rightarrow \chi_{c 1} \pi^{0}$ & $-i 3 \gamma \epsilon^{i j k} \varepsilon^{i}\left(\chi_{c 1}^{\prime}\right) \varepsilon^{j}\left(\chi_{c 1}\right) q^{k} B_{d u}$ \\
$\chi_{c 1}^{\prime} \rightarrow \chi_{c 2} \pi^{0}$ & $3 \sqrt{2} i \gamma \varepsilon^{i}\left(\chi_{c 1}^{\prime}\right) \varepsilon^{i j}\left(\chi_{c 2}\right) q^{j} B_{d u}$ \\
$\chi_{c 2}^{\prime} \rightarrow \chi_{c 2} \pi^{0}$ & $-i 6 \gamma \epsilon^{i j k} \varepsilon^{i l}\left(\chi_{c 2}^{\prime}\right) \varepsilon^{j l}\left(\chi_{c 2}\right) q^{k} B_{d u}$ \\
$h_{c}^{\prime} \rightarrow h_{c} \pi^{0}$ & $-i 6 \gamma \epsilon^{i j k} \varepsilon^{i}\left(h_{c}^{\prime}\right) \varepsilon^{j}\left(h_{c}\right) q^{k} B_{d u}$ \\
\hline \hline
\end{tabular}

Table 2: Tree-level amplitudes for the charmonium transitions with the emission of one pion or eta. A factor of $\sqrt{M_{i} M_{f}}$, with $M_{i(f)}$ denoting the mass of the initial (final) charmonium, should be multiplied to all the expressions to account for the non-relativistic normalization of the charmonium fields.

where $\epsilon_{\pi^{0} \eta}$ is the well-known $\pi^{0}-\eta$ mixing angle, which reads to LO in the chiral expansion

$$
\epsilon_{\pi^{0} \eta}=\frac{\sqrt{3}}{4} \frac{m_{d}-m_{u}}{m_{s}-\hat{m}}
$$

with $\hat{m}=\left(m_{u}+m_{d}\right) / 2$ the average mass of the $u$ and $d$ quarks. Using Dashen's theorem [65], one may express the mixing angle in terms of the masses of the Goldstone bosons at LO in CHPT

$$
\epsilon_{\pi^{0} \eta}=\frac{1}{\sqrt{3}} \frac{M_{K^{0}}^{2}-M_{K^{+}}^{2}+M_{\pi^{+}}^{2}-M_{\pi^{0}}^{2}}{M_{\eta}^{2}-M_{\pi^{0}}^{2}}=0.01 .
$$

The mixing of the $\pi^{0}$ or $\eta$ with the $\eta^{\prime}$ is not considered since it is of higher order. The reason is that the $\eta^{\prime}$ is not a Goldstone boson of the spontaneous breaking of $\mathrm{SU}(3)_{L} \times \mathrm{SU}(3)_{R}$ to the vector subgroup $\mathrm{SU}(3)_{V}$ and its mass as a large scale provides a suppression.

The tree-level amplitudes for the charmonium transitions with the emission of one pion or eta are listed in Table $2^{3}$ where we have defined $B_{d u}=B_{0}\left(m_{d}-m_{u}\right) / F$ and $B_{s l}=$ $B_{0}\left(m_{s}-\hat{m}\right) / F$. A factor of $\sqrt{M_{i} M_{f}}$, with $M_{i(f)}$ denoting the mass of the initial (final) charmonium, should be multiplied to all the expressions to account for the non-relativistic normalization of the charmonium fields used in the effective Lagrangians.

\section{Decay amplitudes from charmed meson loops}

\subsection{Charmed meson loops}

In this section, we list all the possible loops (i.e. the triangle graphs) with the lowest-lying pseudoscalar and vector charmed mesons for each transition. There are three charmed mesons in each loop. To be specific, we denote the one connecting the initial charmonium and the

\footnotetext{
${ }^{3}$ We have checked that the ratios among the spin-averaged absolute square of the transition amplitudes for the transitions between $P$-wave charmonia given in Ref. [58] can be reproduced.
} 


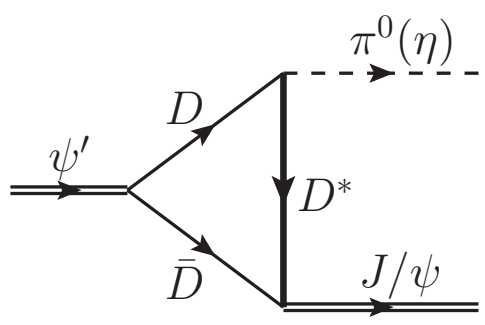

(a)

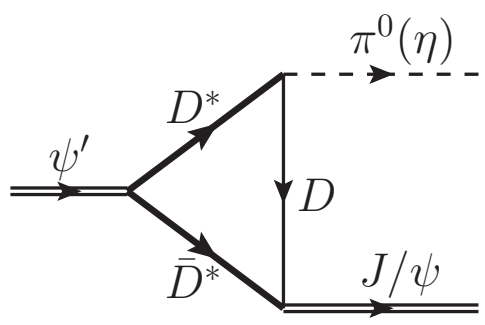

(d)

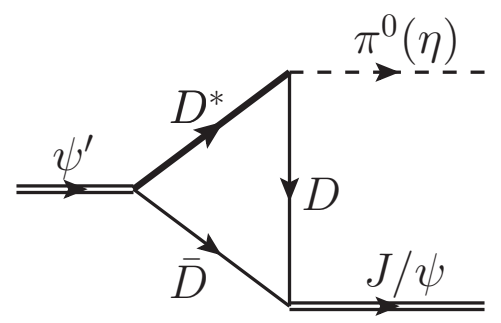

(b)

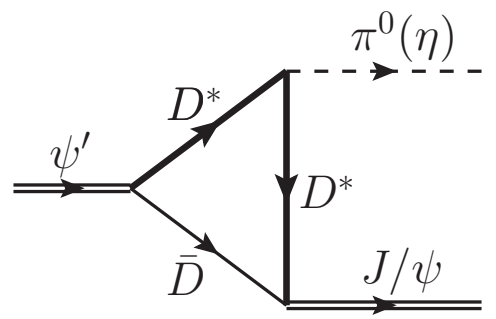

(e)

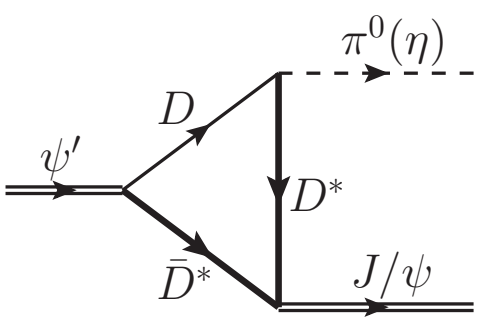

(c)

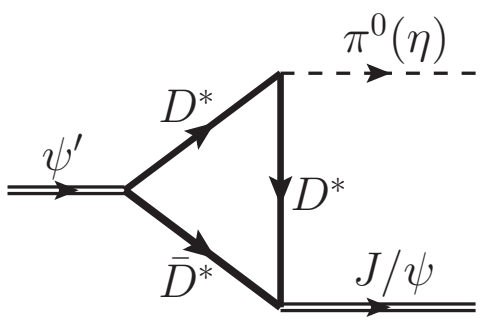

$(\mathbf{f})$

Figure 3: The decays $\psi^{\prime} \rightarrow J / \psi \pi^{0}(\eta)$ through triangle charmed-meson loops. Charmonia, light mesons, pseudoscalar and vector charmed mesons, are denoted by double, dashed, thin and thick solid lines, respectively.

light meson as $M 1$, the one connecting two charmonia as $M 2$, and the one connecting the final charmonium and the light meson as $M 3$. The meson $M i$ has a mass $m_{i}$. For instance, in Fig. 3 which shows all loops contributing to the $\psi^{\prime} \rightarrow J / \psi \pi^{0}(\eta), M 1, M 2$ and $M 3$ are the $D, \bar{D}$ and $D^{*}$, respectively in diagram (a). All the loops contributing to each decay are listed in Table 3 ,

\subsection{Leading order effective Lagrangians}

In order to calculate the leading contributions from the charmed meson loops, we need the LO effective Lagrangians for the couplings. Because the pion and eta are pseudo-Goldstone bosons of the spontaneous chiral symmetry breaking of QCD, their coupling to the charmed mesons in the low-energy limit is constrained by chiral symmetry. The effective Lagrangians were constructed considering both the heavy quark symmetry and chiral symmetry in Refs. [66, 67, 68] (for a review, we refer to Ref. [69]). In the two-component notation of Ref. [56], the charmed mesons are represented by $H_{a}=\vec{V}_{a} \cdot \vec{\sigma}+P_{a}$, with $V_{a}$ and $P_{a}$ denoting the vector and pseudoscalar charmed mesons, respectively, $\vec{\sigma}$ are the Pauli matrices, and $a$ is the light flavor index. Explicitly, one can write $P_{a}\left(V_{a}\right)=\left(D^{(*) 0}, D^{(*)+}, D_{s}^{(*)+}\right)$. The lowest order chiral effective Lagrangian for the axial coupling is [56]

$$
\mathcal{L}_{\phi}=-\frac{g}{2}\left\langle H_{a}^{\dagger} H_{b} \vec{\sigma} \cdot \vec{u}_{b a}\right\rangle
$$

where the axial current is $\vec{u}=-\sqrt{2} \vec{\partial} \phi / F+\mathcal{O}\left(\phi^{3}\right)$.

The LO Lagrangian for the coupling of the $S$ - or $P$-wave charmonium fields to the charmed and anti-charmed mesons can be constructed considering parity, charge conjugation and 


\begin{tabular}{l|l}
\hline \hline$\psi^{\prime} \rightarrow J / \psi \pi^{0}(\eta)$ & {$\left[D, \bar{D}, D^{*}\right],\left[D^{*}, \bar{D}, D\right],\left[D, \bar{D}^{*}, D^{*}\right],\left[D^{*}, \bar{D}^{*}, D\right],\left[D^{*}, \bar{D}, D^{*}\right],\left[D^{*}, \bar{D}^{*}, D^{*}\right]$} \\
$\psi^{\prime} \rightarrow h_{c} \pi^{0}$ & {$\left[D, \bar{D}, D^{*}\right],\left[D^{*}, \bar{D}, D^{*}\right],\left[D^{*}, \bar{D}^{*}, D\right],\left[D, \bar{D}^{*}, D^{*}\right],\left[D^{*}, \bar{D}^{*}, D^{*}\right]$} \\
$h_{c} \rightarrow J / \psi \pi^{0}$ & {$\left[D^{*}, \bar{D}, D\right],\left[D^{*}, \bar{D}, D^{*}\right],\left[D, \bar{D}^{*}, D^{*}\right],\left[D^{*}, \bar{D}^{*}, D\right],\left[D^{*}, \bar{D}^{*}, D^{*}\right]$} \\
$\eta_{c}^{\prime} \rightarrow \chi_{c 0} \pi^{0}$ & {$\left[D^{*}, \bar{D}, D\right],\left[D, \bar{D}^{*}, D^{*}\right],\left[D^{*}, \bar{D}^{*}, D^{*}\right]$} \\
$\chi_{c 0} \rightarrow \eta_{c} \pi^{0}$ & {$\left[D, \bar{D}, D^{*}\right],\left[D^{*}, \bar{D}^{*}, D\right],\left[D^{*}, \bar{D}^{*}, D^{*}\right]$} \\
$\chi_{c 0}^{\prime} \rightarrow \chi_{c 1} \pi^{0}$ & {$\left[D, \bar{D}, D^{*}\right],\left[D^{*}, \bar{D}^{*}, D\right]$} \\
$\chi_{c 1}^{\prime} \rightarrow \chi_{c 0} \pi^{0}$ & {$\left[D^{*}, \bar{D}, D\right],\left[D, \bar{D}^{*}, D^{*}\right]$} \\
$\chi_{c 1}^{\prime} \rightarrow \chi_{c 1} \pi^{0}$ & {$\left[D^{*}, \bar{D}, D^{*}\right]$} \\
$\chi_{c 1}^{\prime} \rightarrow \chi_{c 2} \pi^{0}$ & {$\left[D, \bar{D} * D^{*}\right]$} \\
$\chi_{c 2}^{\prime} \rightarrow \chi_{c 1} \pi^{0}$ & {$\left[D^{*}, \bar{D}^{*}, D\right]$} \\
$\chi_{c 2}^{\prime} \rightarrow \chi_{c 2} \pi^{0}$ & {$\left[D^{*}, \bar{D} D^{*}, D^{*}\right]$} \\
$h_{c}^{\prime} \rightarrow h_{c} \pi^{0}$ & {$\left[D^{*}, \bar{D}, D^{*}\right],\left[D, \bar{D}^{*}, D^{*}\right],\left[D^{*}, \bar{D}^{*}, D\right],\left[D^{*}, \bar{D}^{*}, D^{*}\right]$} \\
\hline \hline
\end{tabular}

Table 3: All the loops contributing to each transition. The mesons are listed as $[M 1, M 2, M 3]$. Flavor labels are dropped for simplicity.

spin symmetry. In two-component notation, the one for the $S$-wave charmonia $J / \psi$ and $\eta_{c}$ reads 32 ]

$$
\mathcal{L}_{\psi}=i \frac{g_{2}}{2}\left\langle J^{\dagger} H_{a} \vec{\sigma} \cdot \overleftrightarrow{\partial} \bar{H}_{a}\right\rangle+\text { H.c. }
$$

where $A \overleftrightarrow{\partial} B \equiv A(\vec{\partial} B)-(\vec{\partial} A) B$, and $\bar{H}_{a}=-\vec{V}_{a} \cdot \vec{\sigma}+\bar{P}_{a}$ is the field for anti-charmed mesons [57]. The Lagrangian for the $P$-wave charmonia at LO is [57]

$$
\mathcal{L}_{\chi}=i \frac{g_{1}}{2}\left\langle\chi^{\dagger i} H_{a} \sigma^{i} \bar{H}_{a}\right\rangle+\text { H.c. }
$$

These Lagrangians were introduced in Ref. [70] in four-component notation. The values of $g_{1}$ and $g_{2}$ are twice of those in Ref. [70. 4 The Lagrangians for the coupling of the radial excited charmonia to the charmed and anti-charmed mesons have the same form as Eqs. (28) 29) with the coupling constants changed to those for the excited states $g_{2}^{\prime}$ and $g_{1}^{\prime}$. For later use, we evaluate the traces in Eqs. (28)29), and rewrite the Lagrangians in terms of the meson fields. The Lagrangian for the $J / \psi$ and $\eta_{c}$ is

$$
\begin{aligned}
\mathcal{L}_{\psi}= & i g_{2} \psi^{\dagger i}\left(V_{a}^{j} \overleftrightarrow{\partial^{i}} \bar{V}_{a}^{j}-V_{a}^{i} \overleftrightarrow{\partial^{j}} \bar{V}_{a}^{j}-V_{a}^{j} \overleftrightarrow{\partial^{j}} \bar{V}_{a}^{i}\right)+g_{2} \epsilon^{i j k} \psi^{\dagger i}\left(P_{a} \overleftrightarrow{\partial^{j}} \bar{V}_{a}^{k}-V_{a}^{j} \overleftrightarrow{\partial^{k}} \bar{P}_{a}\right) \\
& +i g_{2} \psi^{\dagger i} P_{a} \overleftrightarrow{\partial^{j}} \bar{P}_{a}+g_{2} \eta_{c}^{\dagger} \epsilon^{i j k} V_{a}^{i} \overleftrightarrow{\partial^{j}} \bar{V}_{a}^{k}+i g_{2} \eta_{c}^{\dagger}\left(V_{a}^{i} \overleftrightarrow{\partial^{i}} \bar{P}_{a}-P_{a} \overleftrightarrow{\partial}^{i} \bar{V}_{a}^{i}\right)+\text { H.c. }
\end{aligned}
$$

The Lagrangian for the $\chi_{c J}$ and $h_{c}$ reads

$$
\begin{aligned}
\mathcal{L}_{\chi}= & i g_{1} \chi_{c 2}^{\dagger i j}\left(V_{a}^{i} \bar{V}_{a}^{j}+V_{a}^{j} \bar{V}_{a}^{i}\right)+\sqrt{2} g_{1} \chi_{c 1}^{\dagger i}\left(V_{a}^{i} \bar{P}_{a}+P_{a} \bar{V}_{a}^{i}\right)+\frac{i}{\sqrt{3}} g_{1} \chi_{c 0}^{\dagger}\left(\vec{V}_{a} \cdot \vec{V}_{a}+3 P_{a} \bar{P}_{a}\right) \\
& -g_{1} \epsilon^{i j k} h_{c}^{\dagger i} V_{a}^{j} \bar{V}_{a}^{k}+i g_{1} h_{c}^{\dagger i}\left(V_{a}^{i} \bar{P}_{a}-P_{a} \bar{V}_{a}^{i}\right)+\text { H.c. },
\end{aligned}
$$

\footnotetext{
${ }^{4}$ In the definition of the Lagrangians of Ref. [70, each term is doubled for heavy quarkonia with the same flavor of quark and anti-quark. Hence the values of the coupling constants there should be half of those in our paper.
} 
where the trace and symmetry properties $\chi_{c 2}^{i j}{ }^{i j}=0$ and $\chi_{c 2}^{i j} \epsilon^{i j k}=0$ have been used in the derivations.

\subsection{Decay amplitudes of the pion or eta emission transitions}

The amplitudes for all the transitions with charged charmed meson loops are listed in Appendix $\mathrm{B}$. The charmonia are isospin and $\mathrm{SU}(3)$ flavor singlets, the pions form an isospin triplet and the $\eta$ is an element of the $\mathrm{SU}(3)$ octet. Therefore, the transitions between two charmonia with the emission of one pion or eta break isospin or SU(3) symmetry. The leading contributions to the eta transition amplitudes are given by the differences between the non-strange and strange charmed meson loops. The decay amplitude for the $\psi^{\prime} \rightarrow J / \psi \eta$ is

$$
\mathcal{M}\left(\psi^{\prime} \rightarrow J / \psi \eta\right)=\frac{1}{\sqrt{3}}\left[\mathcal{M}\left(\psi^{\prime} \rightarrow J / \psi \phi\right)_{0}+\mathcal{M}\left(\psi^{\prime} \rightarrow J / \psi \phi\right)_{ \pm}-2 \mathcal{M}\left(\psi^{\prime} \rightarrow J / \psi \phi\right)_{s}\right]
$$

where $\phi$ denotes the light pseudoscalar meson, and the expression of $\mathcal{M}\left(\psi^{\prime} \rightarrow J / \psi \phi\right)_{ \pm}$is given as Eq. (B.1) in Appendix B. The amplitude with lower index 0 can be obtained by simply replacing the charged charmed mesons in Eq. (B.1) by the neutral ones, and the lower index $s$ denotes the charmed-strange meson loops. The leading contributions to the pionic transition amplitudes are given by the differences between the neutral and charged charmed meson loops, and also from the the $\pi^{0}-\eta$ mixing through the loops contributing to the eta transition. From Eq. (24), the physical pion is

$$
\tilde{\pi}^{0}=\pi^{0}+\epsilon_{\pi^{0} \eta} \eta+\mathcal{O}\left(\epsilon_{\pi^{0} \eta}^{2}\right) .
$$

Hence, the amplitude for the $\psi^{\prime} \rightarrow J / \psi \pi^{0}$ reads

$$
\begin{aligned}
\mathcal{M}\left(\psi^{\prime} \rightarrow J / \psi \pi^{0}\right)= & \frac{\epsilon_{\pi^{0} \eta}}{\sqrt{3}}\left[\mathcal{M}\left(\psi^{\prime} \rightarrow J / \psi \phi\right)_{0}+\mathcal{M}\left(\psi^{\prime} \rightarrow J / \psi \phi\right)_{ \pm}-2 \mathcal{M}\left(\psi^{\prime} \rightarrow J / \psi \phi\right)_{s}\right] \\
& +\mathcal{M}\left(\psi^{\prime} \rightarrow J / \psi \phi\right)_{0}-\mathcal{M}\left(\psi^{\prime} \rightarrow J / \psi \phi\right)_{ \pm} .
\end{aligned}
$$

Note that although the loop amplitudes $\mathcal{M}\left(\psi^{\prime} \rightarrow J / \psi \phi\right)_{0, \pm, s}$ take the same form as those in Eq. (32), the momentum $q$ is different for the different decays, it is given by the threemomentum of the light meson in the final state. For all the other transitions considered in this paper, the available phase spaces only allow the emission of one pion, and the amplitudes can be obtained in a similar way to Eq. (34) using the equations given in Appendix B.

As can be seen from the presence of a Pauli matrix between two charmonium fields in the Lagrangians $\mathcal{L}_{S S}$ and $\mathcal{L}_{S P}$, the transitions between the $S$-wave charmonia violate the heavy quark spin symmetry, so do those between one $S$ and one $P$-wave charmonia. Were the heavy quark spin symmetry exact, the vector and pseudoscalar charmed mesons would have the same mass. In the heavy quark spin symmetric world, all the meson-loop amplitudes for the transitions between two $S$-wave charmonia, and those between one $S$ and one $P$-wave charmonia would vanish. This is because the contributions from different loops would cancel with each other completely as one may easily see from the amplitudes listed in Appendix B by putting $M_{D}=M_{D^{*}}$. This means the vector and pseudoscalar heavy mesons have to be considered simultaneously to keep the structure of the spin symmetry which is used in constructing the Lagrangians and relating the coupling constants for different transitions. The transitions between the $P$-wave charmonia respect the spin symmetry, so the resulting amplitudes do not 
vanish in the given heavy quark spin symmetric world as shown in Appendix B.3. Keeping spin symmetry structure of the tree-level amplitudes might be a general feature of the heavy hadron loops, so that the analysis of the spin partner of heavy hadron molecules in Ref. [71], which is based on the spin symmetry without considering loops, would not be affected.

\subsection{Power counting of the loops}

Before proceeding to numerical calculations, one must analyze the power counting of the loops in the NREFT formalism. Any loop diagram in the paper is composed of two vertices for the coupling of a charmonium to charmed and anti-charmed mesons - both characterized by a vertex structure and a coupling constant, one vertex for the coupling of a pion or eta to charmed mesons, and three propagators for charmed mesons. The power counting of a given diagram is obtained via estimating each individual ingredient by a typical value. Based on this analysis, each diagram can be assigned a definite order $n$ in the given expansion parameter, which for the transitions at hand turn out to be the velocity $v$ of the intermediate heavy mesons. Once a complete calculation up to order $n$ is performed, the uncertainty of the calculation may be estimated as $v^{(n+1)}$ — we will show below that additional scales introduced by the dimensionful coupling constants do not distort this picture.

Since effective field theories are in general non-renormalizable, they have to be regularized and renormalized order by order. A consistent power counting thus has to guarantee that at each order where there are divergences there are also appropriate counterterms available. In this section we check this for the transitions and the power counting at hand.

Let us first focus on the propagators, and take the scalar loop integral as an example. The scalar loop integral in $d$ dimensions for the triangle graphs under consideration is defined as

$$
I(q)=\int \frac{d^{d} l}{(2 \pi)^{d}} \frac{i}{D}=\int \frac{d^{d} l}{(2 \pi)^{d}} \frac{i}{\left(l^{2}-m_{1}^{2}+i \epsilon\right)\left[(P-l)^{2}-m_{2}^{2}+i \epsilon\right]\left[(l-q)^{2}-m_{3}^{2}+i \epsilon\right]},
$$

where $P$ is the momentum of the initial charmonium and $q$ is the momentum of the light meson in the final state. Non-relativistically, in the rest-frame of the initial charmonium, the loop can be written as

$$
\begin{aligned}
I(q) & =\frac{i}{8 m_{1} m_{2} m_{3}} \int \frac{d^{4} l}{(2 \pi)^{4}} \frac{1}{\left(l^{0}-T_{1}(|\vec{l}|)\right)\left(P^{0}-l^{0}-T_{2}(|\vec{l}|)\right)\left(l^{0}-q^{0}-T_{3}(|\vec{l}-\vec{q}|)\right)} \\
& =\frac{1}{8 m_{1} m_{2} m_{3}} \int \frac{d^{3} l}{(2 \pi)^{3}} \frac{1}{\left(E_{i}-T_{2}(|\vec{l}|)-T_{1}(|\vec{l}|)\right)\left(E_{f}-T_{2}(|\vec{l}|)-T_{3}(|\vec{l}-\vec{q}|)\right)}
\end{aligned}
$$

where $T_{i}(p)=p^{2} / 2 m_{i}=m_{i} v^{2} / 2$, with $v$ being the charmed meson velocity, denotes the kinetic energy for the charmed mesons with masses $m_{1}, m_{2}$ and $m_{3}, E_{i}=M_{i}-m_{1}-m_{2}$ and $E_{f}=M_{f}-m_{2}-m_{3}-E_{\pi}$ denote the energies available for the first (before the pion emission) and second (after the pion emission) two-heavy-meson intermediate state. One may assign the charmed meson momentum as $M_{D} v$. Here, we will only count the power of $v$ since the dimension of the loops can be simply implemented by multiplying proper power of $M_{D}$. Thus, the scalar loop scales as $1 /(16 \pi)\left[v^{3} /\left(v^{2}\right)^{2}\right]=1 /(16 \pi v)$, since in the last line of Eq. (36) each of the non-relativistic propagators is counted as $1 / v^{2}$ and the integral measure is counted as $v^{3} /(16 \pi)$, where it was used that the loops are dominated by the unitarity 
cut, which produces a factor of $\pi$ to be combined with the standard factor $1 /(4 \pi)^{2}$ from the integral measure. This factor is common to all loop contributions as given in Table 4 .

As indicated in the Eq. (27) for the axial-coupling of the pion or eta to the charmed mesons, the corresponding vertex is proportional to the external momentum of the pion or eta, denoted by $q$ - this gives one power of $q$ in the expressions for all the loop contributions in Table 4. Further, we have to account for the isospin or $\mathrm{SU}(3)$ violation as well as the momentum dependence of the charmonium-charmed meson vertices - the scaling of the coupling constants will be discussed below. To account for the corresponding symmetry breaking, in each power counting estimate for the loops listed in Table 4, we have pulled out the meson mass difference, denoted as $\Delta 5$ which is a small energy scale, and divided $\Delta$ by a factor which characterizes the intrinsic energy, $v^{2}$, for balance. The vertices are more complicated since their scaling behavior depends on the quantum numbers of the charmonia in both the initial and final states. We therefore classify the vertices into three groups:

1) Both the vertices for the initial and final charmonia are in $S$-wave. This corresponds to the transitions between two $P$-wave charmonia (denoted by $P P$ in Table 4). In this case, the vertices do not give any non-trivial contribution to the power counting, and they scale as $\mathcal{O}\left(v^{0} q^{0}\right)=\mathcal{O}(1)$.

2) One vertex is in $S$-wave, and the other one is in $P$-wave. This corresponds to the transitions between one $S$-wave and one $P$-wave charmonia (denoted by $S P$ in Table 4 ). In this case, the loop momentum must be contracted with the external momentum $q$, and hence render the scale of the $P$-wave vertex to be $\mathcal{O}(q)$. In this case a factor $1 / M_{D}^{2}$ needs to be introduced to match dimensions.

3) Both the vertices are in $P$-wave. This corresponds to the transitions between two $S$-wave charmonia (denoted by $S S$ in Table 4). In this case the loops are tensor loops, which can be split into two parts as given in Eq. (A.5). The part $q^{i} q^{j} I_{0}^{(2)}(q)$ scales as two powers of the eternal momentum, i.e., $\mathcal{O}\left(q^{2}\right)$. In the other part, the two momenta in the numerator of the loop integrand contract with each other, and as a result, the Kronecker delta appears. Here all momenta appearing are internal momenta, which, by assumption, scale as $v$. Thus, for this piece of the integral the two vertex functions together scale as $v^{2}$. In the transitions considered in the paper, one always has $q \lesssim M_{D} v$. Hence, the product of the two vertices in this case can be counted as $\mathcal{O}\left(v^{2}\right)$.

The last ingredients to be discussed are the coupling constants $g, g_{1}$ and $g_{2} . g$ is the axial coupling constant for the heavy mesons. It is dimensionless, and should be of order unity. Based on the underlying Lagrangians, e.g., the dimension of $g_{2}$, the coupling of the $S$-wave charmonia to the open charm ground states, is $-3 / 2$. In Ref. [70], using vector meson dominance arguments, the authors obtain $g_{2}=\sqrt{M_{J / \psi}} /\left(M_{D} f_{J / \psi}\right)$, where $f_{J / \psi}$ is the decay constant of the $J / \psi$. On the quark level it scales with the $J / \psi$ wave function at the origin that, on dimensional grounds, should be $f_{J / \psi} \sim m_{c} v_{c}^{3 / 2}$ — the quark mass and velocity $m_{c}$ and $v_{c}$ were introduced at the end of Section 2.1. Hence, we have

$$
g_{2} \sim \frac{\sqrt{2}}{\left(m_{c} v_{c}\right)^{3 / 2}} .
$$

\footnotetext{
${ }^{5}$ These meson mass differences are, of course, generated by quark mass differences and e.m. effects. For the charmed mesons, $\Delta$ is of similar size as the quark mass differences $\delta$.
} 
Using $m_{c}=1.5 \mathrm{GeV}$ and $v_{c}^{2}=0.3$ we get $g_{2}=1.9 \mathrm{GeV}^{-3 / 2}$, which is close to independent model estimates in a range of $2.1 \ldots 2.9 \mathrm{GeV}^{-3 / 2}$ for this quantity existing in the literature [85, 86, 87].

The expression for $g_{1}$ derived from vector meson dominance is $g_{1}=-2 \sqrt{M_{\chi_{c 0}} / 3} / f_{\chi_{c 0}}[70]$. From dimensional analysis, the decay constant of the $P$-wave charmonium $\chi_{c 0}$ should scale with the first derivative of the wave function at the origin. Hence,

$$
g_{1} \sim-\sqrt{\frac{2}{3}} \frac{2}{\sqrt{m_{c}} v_{c}^{5 / 2}} .
$$

Using the above scaling, we can work out the power counting of the loops for different processes.For the $S S$ transitions, as mentioned at the end of Section 3.3, the loop amplitudes vanish in case of $M_{D}=M_{D^{*}}$, i.e., the loop amplitude violates spin symmetry as the tree level amplitude does. So a factor of $\Lambda_{\mathrm{QCD}} / m_{c}$ should also be considered in the scaling of the loop amplitude as well as the estimate for the loop integral itself, $1 /(16 \pi v)$, and a factor $v^{2}$, which originates from the two decay vertices. Furthermore, the nonrelativistic normalization factor $\sqrt{M_{i} M_{f}} m_{1} m_{2} m_{3}$ gives a factor $2 m_{c}^{4}$. As explained in the above, a factor of $\Delta /\left(m_{D} v^{2}\right) \sim$ $\Delta /\left(m_{c} v^{2}\right)$ should also be introduced to account for the isospin breaking. Collecting all factors, we get for the scaling of the loop amplitude for the $S S$ transitions

$$
\begin{aligned}
\mathcal{M}_{\text {loop }}^{S S} & \sim \frac{g}{F} g_{2} g_{2}^{\prime} q 2 m_{c}^{4} \frac{v^{2}}{16 \pi v} \frac{\Lambda_{\mathrm{QCD}}}{m_{c}} \frac{\Delta}{m_{c} v^{2}} \\
& \sim \frac{1}{4 \pi v_{c}^{3}} \frac{1}{m_{c}} \frac{q \Delta}{v} .
\end{aligned}
$$

As will be shown later, these rules for power counting of the loops are satisfied by explicit non-relativistic calculations.

Similarly, for the $S P$ and $P P$ transitions, using the scaling of $g_{1}$ given in Eq.(38) we have

$$
\begin{aligned}
\mathcal{M}_{\text {loop }}^{S P} & \sim \frac{g}{F} g_{1} g_{2} q 2 m_{c}^{4} \frac{1}{16 \pi v} \frac{q}{M_{D}^{2}} \frac{\Lambda_{\mathrm{QCD}}}{m_{c}} \frac{\Delta}{m_{c} v^{2}} \\
& \sim \frac{1}{2 \sqrt{3} \pi v_{c}^{4}} \frac{q^{2} \Delta}{M_{D}^{2} v^{3}}
\end{aligned}
$$

and

$$
\begin{aligned}
\mathcal{M}_{\text {loop }}^{P P} & \sim \frac{g}{F} g_{1} g_{1}^{\prime} q 2 m_{c}^{4} \frac{1}{16 \pi m_{c}^{2} v} \frac{\Delta}{m_{c} v^{2}} \\
& \sim \frac{1}{3 \pi v_{c}^{5}} \frac{1}{\Lambda_{\mathrm{QCD}}} \frac{q \Delta}{v^{3}} .
\end{aligned}
$$

In the last equation, a factor of $1 / M_{D}^{2} \sim 1 / m_{c}^{2}$ was taken into account to give the correct dimension of the scalar loop. This can be done as mentioned below Eq. (36).

The scaling for the loop amplitudes in Eqs. (39), (40) and (41) need to be compared to the corresponding estimates for the tree-level amplitudes. The relevant estimate is given explicitly in Eq. (18) for the $S S$ transitions and Eq. (19) for the $P P$ and $S P$ transitions - the final results for all transitions are given in Table 4. For charmed mesons, $\Delta$ is of similar size as $\delta$ as may be checked numerically, i.e. $M_{D^{+}}-M_{D^{0}} \simeq m_{d}-m_{u}$ and $M_{D_{s}^{+}}-M_{D^{+}} \simeq m_{s}-m_{d}$. At this stage, let us make a remark about the factor containing the charm quark velocity 


\begin{tabular}{|c|c|c|}
\hline & Tree-level & Loops \\
\hline$S S$ & $\frac{1}{m_{c}} q \delta$ & $\frac{\mathcal{N}}{m_{c}}\left(\frac{v^{3}}{v^{4}}\right) v^{2} q\left(\frac{\Delta}{v^{2}}\right)=\frac{\mathcal{N}}{m_{c}} \frac{q \Delta}{v}$ \\
$S P$ & $\delta$ & $\mathcal{N}\left(\frac{v^{3}}{v^{4}}\right) \frac{q q}{M_{D}^{2}}\left(\frac{\Delta}{v^{2}}\right)=\mathcal{N} \frac{q^{2}}{v^{3} M_{D}^{2}} \Delta$ \\
$P P$ & $\frac{1}{\Lambda_{\mathrm{QCD}}} q \delta$ & $\frac{\mathcal{N}}{\Lambda_{\mathrm{QCD}}}\left(\frac{v^{3}}{v^{4}}\right) q\left(\frac{\Delta}{v^{2}}\right)=\frac{\mathcal{N}}{\Lambda_{\mathrm{QCD}}} \frac{q \Delta}{v^{3}}$ \\
\hline
\end{tabular}

Table 4: Power counting of the tree-level amplitudes and the (leading) loops. Here $S S, S P$ and $P P$ represent transitions between two $S$-wave, one $S$-wave and one $P$-wave, and two $P$-wave charmonia, respectively. The parameter $\delta$ denotes the quark mass differences, and $\Delta$ the charmed meson mass differences. They are the strength parameters for isospin or $\mathrm{SU}(3)$ symmetry violation. $v$ is the heavy-meson velocity in the intermediate loops, $q$ the momentum of the outgoing pseudoscalar meson, and $M_{D}$ the mass of the heavy mesons in the loop. For the origin of the individual factors, see the text. $\mathcal{N}=1 /\left(4 \pi v_{c}^{3}\right), 1 /\left(2 \sqrt{3} \pi v_{c}^{4}\right)$ and $1 /\left(3 \pi v_{c}^{5}\right)$ for the $S S, S P$ and $P P$ transitions, respectively, where $v_{c}$ denotes the charm quark velocity inside the charmonia.

scaling of the coupling constants, i.e. $\mathcal{N}=1 /\left(4 \pi v_{c}^{3}\right), 1 /\left(2 \sqrt{3} \pi v_{c}^{4}\right)$ and $1 /\left(3 \pi v_{c}^{5}\right)$ for the $S S$, $S P$ and $P P$ transitions, respectively. Taking $v_{c}=\sqrt{0.3}$, we get $\mathcal{N}=0.5,1$ and 2 for the SS, SP and PP transitions, respectively. All these numbers are of order unity. Although $\mathcal{N}$ scales differently in powers of $v_{c}$ for different processes, the numerical values are not very different. This is because the charm quark velocity $v_{c}$ in such a charmonium is not so small. Hence the numerical values of the presumably well-separated scales may be similar in practice. Sometimes, the order of the scales is even reversed. For instance, purely from the scaling, $f_{J / \psi} \sim m_{c} v_{c}^{3 / 2}$ should be larger than $f_{\chi_{c 0}} \sim m_{c} v_{c}^{5 / 2}$. However, using the experimental value $\Gamma\left(J / \psi \rightarrow e^{+} e^{-}\right)=5.55 \pm 0.14 \mathrm{keV}$ [4], and the relation between decay constant and the leptonic width of the $J / \psi$

$$
\Gamma\left(J / \psi \rightarrow e^{+} e^{-}\right)=\frac{16 \pi}{27} \frac{\alpha^{2}}{M_{J / \psi}} f_{J / \psi}^{2},
$$

$f_{J / \psi}=416 \mathrm{MeV}$, while the numerical result from QCD sum rules gives $f_{\chi_{c 0}}=510 \pm$ $40 \mathrm{MeV}$ [72. Furthermore, in view that there must be unknown numerical factors in the coupling constants $g_{1}, g_{2}$ and also the the tree-level ones, and the $v_{c}$ scaling of tree-level couplings is not known yet as mentioned before Eq. (16), we will neglect the subtlety caused by $\mathcal{N}$ in the power counting, and just take it to be unity for all the transitions (numerical calculations in the next section will not be affected). Based on the underlying power counting, we therefore predict loops to be enhanced by a power of $1 / v$ for $S S$ and $1 / v^{3}$ for $P P$ transitions, while for $S P$ transitions in many cases loops appear to be suppressed - see detailed discussion below. One should also keep in mind that the meson velocity $v$ in some of the processes to be discussed is as large as 0.5 so that $1 / v$ for the $S S$ transitions might 
be in practice not a large enhancement. But it should be sufficient to say that the the loops are important compared to the tree-level contributions, and should not be neglected in any realistic analysis.

As mentioned above, a consistent power counting needs to ensure that all appearing divergences can be absorbed into appropriate counterterms. This is of importance here since the leading counterterms are supplied by the tree-level amplitudes discussed above, which in some cases appear in higher orders than the leading loops. In such a case, they might get renormalized by absorbing the divergence of the loops. Although the scalar loop, defined in Eq. (35) is convergent, for some transitions there are momentum factors at the vertices, resulting in divergent integrals. In Appendix $B$ the contributions to all transitions are expressed in terms of a few fundamental integrals defined in Appendix $\mathrm{A}$. Note that, in order to account for the non-relativistic normalization of the charmonium and charmed meson fields, one needs to multiply each amplitude by a factor of $\sqrt{M_{i} M_{f}} m_{1} m_{2} m_{3}$, where $M_{i(f)}$ is the mass of the initial (final) charmonium, and $m_{i}(i=1,2,3)$ are the masses of the charmed mesons in the loops. The loop functions $I^{(1)}(q), I_{0}^{(2)}(q)$ and $I_{1}^{(2)}(q)$ are constructed from the basic scalar loop functions $I(q)$ defined in Eq. (35)

$$
q^{i} I^{(1)}(q)=i \int \frac{d^{d} l}{(2 \pi)^{d}} \frac{l^{i}}{D}, \quad q^{i} q^{j} I_{0}^{(2)}(q)+\delta^{i j} \vec{q}^{2} I_{1}^{(2)}(q)=i \int \frac{d^{d} l}{(2 \pi)^{d}} \frac{l^{i} l^{j}}{D}
$$

and $B(c)$, which in $d$ space-time dimensions is

$$
B(c)=4 \pi N \int \frac{d^{d-1} l}{(2 \pi)^{d-1}} \frac{1}{\vec{l}^{2}+c-i \epsilon}
$$

where $N=\mu_{12} \mu_{23} /\left(16 \pi m_{1} m_{2} m_{3}\right)$ with $\mu_{i j}=m_{i} m_{j} /\left(m_{i}+m_{j}\right)$ for the reduced masses. For later use, we define the following quantities

$$
a=\left(\frac{\mu_{23}}{m_{3}}\right)^{2} \vec{q}^{2}, \quad c=2 \mu_{12} b_{12}, \quad c^{\prime}=2 \mu_{23} b_{23}+\frac{\mu_{23}}{m_{3}} \vec{q}^{2}
$$

where $b_{12}=m_{1}+m_{2}-M_{i}$, and $b_{23}=m_{2}+m_{3}+q^{0}-M_{i}$.

In our full calculation all integrals are evaluated using dimensional regularization. In this section, however, in order to make all divergences explicit, we investigate the divergence structure by introducing a sharp momentum cut-off in three dimensions. With this one finds

$$
B(c)=4 \pi N \int^{\Lambda} \frac{d^{3} l}{(2 \pi)^{3}} \frac{1}{\vec{l}^{2}+c-i \epsilon}=N\left\{\frac{2 \Lambda}{\pi}-\sqrt{c-i \epsilon}+\mathcal{O}\left(\frac{1}{\Lambda}\right)\right\} .
$$

The non-analytic part is finite. It is determined by unitarity, and hence does not depend on the choice of regularization method. In the integral $I^{(1)}$ only the linear combination $B\left(c^{\prime}-a\right)-B(c)$ appears - c.f. Eq. (A.6) - and thus the divergence of $B(c)$ cancels. The $\mathrm{UV}$ divergent part of $\vec{q}^{2} I_{0,1}^{(2)}(q)$ is

$$
\vec{q}^{2} I_{0}^{(2)}(q)^{\mathrm{UV}}=\vec{q}^{2} I_{1}^{(2)}(q)^{\mathrm{UV}}=\frac{N}{\pi} \Lambda
$$

For the loop amplitudes given in Appendix B, only the transitions between two $S$-wave charmonia, i.e. $\psi^{\prime} \rightarrow J / \psi \pi^{0}(\eta)$, are divergent. 
In order to estimate the finite parts of the pertinent integrals we may use $a \ll c \approx c^{\prime} \approx$ $\sqrt{2 \mu b} \approx M_{D} v$ and expand the expressions in a series around $a=0$. With this we find

$$
\begin{aligned}
B\left(c^{\prime}-a\right) & =B(c)+\mathcal{O}(a)=-N \sqrt{c}+\mathcal{O}(a), \\
I(q) & \approx N \frac{1}{\sqrt{c}}+\mathcal{O}(a), \\
I^{(1)}(q) & \approx N \frac{\mu_{23}}{m_{3}} \frac{1}{2 \sqrt{c}}+\mathcal{O}(a), \\
\vec{q}^{2} I_{1}^{(2)}(q) \text { finite } & \approx-N \sqrt{c}+\mathcal{O}(a), \\
\vec{q}^{2} I_{0}^{(2)}(q) & =\mathcal{O}(a) .
\end{aligned}
$$

The exact expressions are given in Appendix $\mathrm{A}$.

As an example we now focus on the analysis of diagram (b) in Fig. 3 to the amplitude of the $\psi^{\prime} \rightarrow J / \psi \pi^{0}$ — the discussion is easily generalized to the other diagrams. The amplitude reads

$$
\mathcal{M}\left(\psi^{\prime} \rightarrow J / \psi \pi^{0}\right)_{(\mathrm{b})}=N_{(\mathrm{b})}\left[\vec{q}^{2} I_{1}^{(2)}\left(q, D^{* 0}, D^{0}, D^{0}\right)-\vec{q}^{2} I_{1}^{(2)}\left(q, D^{* \pm}, D^{ \pm}, D^{ \pm}\right)\right],
$$

with $N_{(\mathrm{b})}=-4(g / F) g_{2} g_{2}^{\prime} \epsilon^{i j k} q^{i} \varepsilon^{j}\left(\psi^{\prime}\right) \varepsilon^{k}(J / \psi)$. The contribution of the finite part of the loop function $I_{1}^{(2)}(q)$ to diagram (b) behaves as

$$
\mathcal{M}\left(\psi^{\prime} \rightarrow J / \psi \pi^{0}\right)_{(\mathrm{b})}^{\text {finite }} \sim-N_{(\mathrm{b})}\left(N_{n} \sqrt{2 \mu_{n} b_{n}}-N_{c} \sqrt{2 \mu_{c} b_{c}}\right),
$$

where the lower index $n$ means neutral, and $c$ charged. Denoting the mass difference between the charged and neutral charmed mesons by $\Delta, 6$ we have $\mu_{c}=\mu_{n}+\Delta / 2$ and $b_{c}=b_{n}+2 \Delta$. Thus, we have

$$
\begin{aligned}
\mathcal{M}\left(\psi^{\prime} \rightarrow J / \psi \pi^{0}\right)_{(\mathrm{b})}^{\text {finite }} & \sim N_{(\mathrm{b})} N \Delta \frac{2 \mu_{n}+b_{n} / 2}{\sqrt{2 \mu_{n} b_{n}}}+\mathcal{O}\left(\Delta^{2}\right) \\
& \sim N_{(\mathrm{b})} N \frac{\Delta}{v}
\end{aligned}
$$

which is consistent with the power counting analysis for $S S$ transitions given above. Here, we neglect the difference between $N_{n}$ and $N_{c}$ since it is of higher order. On the other hand, the UV divergence of diagram (b) is

$$
\begin{aligned}
\mathcal{M}\left(\psi^{\prime} \rightarrow J / \psi \pi^{0}\right)_{(\mathrm{b})}^{\mathrm{UV}} & =N_{(\mathrm{b})} \frac{\Lambda}{\pi}\left(N_{n}-N_{c}\right) \\
& \sim N_{(\mathrm{b})} N \frac{\Lambda}{\mu_{n}} \frac{\Delta}{\pi}
\end{aligned}
$$

Therefore, for diagram (b), the finite part is of order $\mathcal{O}\left(\Delta v^{-1}\right)$, while the UV divergence is of order $\mathcal{O}\left(\Delta v^{0}\right)$. Hence the UV divergence is one order higher in the expansion of $v$. Recalling the tree-level contribution to the $\psi^{\prime} \rightarrow J / \psi \pi^{0}$ starts from the same order as $\mathcal{O}\left(\Delta v^{0}\right)-$

\footnotetext{
${ }^{6}$ We neglect the difference between the mass difference for the pseudoscalar mesons and that for the vector ones, which is of higher order in heavy quark expansion. Empirically, one finds $\Delta_{P}=m_{D^{ \pm}}-m_{D^{0}}=$ $4.77 \pm 0.10 \mathrm{MeV}$, and $\Delta_{V}=m_{D^{* \pm}}-m_{D^{* 0}}=3.29 \pm 0.21 \mathrm{MeV}$. Their difference is about $30 \%$ of $\Delta_{P}$, which can be understood as $\mathcal{O}\left(\Lambda_{\mathrm{QCD}} / m_{c}\right)$ effects.
} 
see the column for $S S$ transitions in Table 4 - such a divergence can be renormalized by a counterterm in the Lagrangian for the tree-level contribution.

As a result of this analysis we summarize, comparing the loop contributions with the tree-level decay amplitudes given in the last section and in Table 4, where we assume the same scale for the light quark mass and the heavy meson mass differences, $\delta \simeq \Delta[27$, that the loop contributions for the $S S$ transitions are enhanced by a factor of $1 / v \approx 2$, and for the $P P$ transitions even by a factor of $1 / v^{3} \approx 10$. The situation for the $S P$ transitions should be analyzed case by case since an enhancement factor $1 / v^{3}$ competes with a suppression factor $q^{2} / M_{D}^{2}$. For the $S P$ transitions with small phase space, the external momentum $q$ might be small enough to make $q^{2} /\left(v^{3} M_{D}^{2}\right)$ much smaller than 1 , which is satisfied for the decays $\psi^{\prime} \rightarrow h_{c} \pi^{0}$ and $\eta_{c}^{\prime} \rightarrow \chi_{c 0} \pi^{0}$ [48]. In this case, the decay is dominated by the tree-level contributions. However, for the decays with external momentum $q \gtrsim M_{D} v^{3 / 2}$, the factor $q^{2} /\left(v^{3} M_{D}^{2}\right) \gtrsim 1$, so is no more a suppression, and hence the tree-level contributions are at least as important as the loop contributions. In summary, our power counting was shown to be consistent with the divergence structure of the pertinent integrals.

\section{Results for the decay widths}

In this section, we give the results from explicit calculations of all the mentioned transitions with emphasis on the contributions from charmed meson loops. In the numerical evaluations we use the following values for the meson masses [44]

$$
\begin{array}{rlrl}
M_{\pi^{0}} & =134.98 \mathrm{MeV}, & M_{\eta}=547.85 \mathrm{MeV}, & \\
M_{D^{+}}=1869.60 \mathrm{MeV}, & M_{D^{0}}=1864.83 \mathrm{MeV}, & M_{D_{s}}=1968.47 \mathrm{MeV}, \\
M_{D^{*+}}=2010.25 \mathrm{MeV}, & M_{D^{* 0}}=2006.96 \mathrm{MeV}, & M_{D_{s}^{*}}=2112.3 \mathrm{MeV} \\
M_{J / \psi} & =3096.92 \mathrm{MeV}, & M_{\eta_{c}}=2980.3 \mathrm{MeV}, & M_{\psi^{\prime}}=3686.09 \mathrm{MeV} \\
M_{\eta_{c}^{\prime}}=3637 \mathrm{MeV}, & M_{h_{c}}=3525.42 \mathrm{MeV}, & M_{\chi_{c 0}}=3414.75 \mathrm{MeV} \\
M_{\chi_{c 1}}=3510.66 \mathrm{MeV}, & M_{\chi_{c 2}}=3556.20 \mathrm{MeV}, & M_{\chi_{c 2}^{\prime}}=3929 \mathrm{MeV}
\end{array}
$$

Here we have identified the $\chi_{c 2}^{\prime}$ with the $Z(3930)$ as done by the Particle Data Group, see e.g. Refs. [73, 74, 6, 50]. The $Z(3930)$ was observed in the $D \bar{D}$ mass distribution in photonphoton collisions by the Belle Collaboration [73] with a mass of $3929 \pm 5 \pm 2 \mathrm{MeV}$ and width of $29 \pm 10 \pm 2 \mathrm{MeV}$. The observed angular distribution suggests that its quantum numbers are $J^{P C}=2^{++}$. The widths of the $\chi_{c 2}^{\prime}$ calculated in quark models are consistent with the observed values for the $Z(3930)$ [74, 50].

There is no unambiguous candidate for either of the other three excited $P$-wave charmonia $\chi_{c 0}^{\prime}, \chi_{c 1}^{\prime}$ and $h_{c}^{\prime}$. Very recently, the Belle Collaboration observed an enhancement, called the $X(3915)$, in the $J / \psi \omega$ mass distribution in photon-photon collisions [75]. The mass and width of the $X(3915)$ were reported to be $3915 \pm 3 \pm 2$ and $17 \pm 10 \pm 3$, respectively [75]. In Ref. [76], the authors suggest the $X(3915)$ as the $\chi_{c 0}^{\prime}$. However, this assignment may be criticized from the following points: First, since the $\chi_{c 0}^{\prime}$ is above the $D \bar{D}$ threshold, and it couples to $D \bar{D}$ in an $S$-wave, one would expect it to have a larger width than the $17 \mathrm{MeV}$ of the $X(3915)$. Second, were the $Z(3930)$ the $\chi_{c 2}^{\prime}$, the mass difference $M_{Z(3930)}-M_{X(3915)}=14 \pm 6 \mathrm{MeV}$ is too small for the hyperfine splitting, since the mass splitting between the ground state $\chi_{c 2}$ and $\chi_{c 0}$ is one order of magnitude larger, $M_{\chi_{c 2}}-M_{\chi_{c 0}}=141 \mathrm{MeV}$ [44]. In addition, one expects the hyperfine splittings for the bottomonia are smaller than the corresponding 
ones for the charmonia, as can be checked from all the measured cases. The mass splitting between the excited $P$-wave bottomonia $\chi_{b 2}^{\prime}$ and $\chi_{b 0}^{\prime}$ is $36.2 \pm 0.8 \mathrm{MeV}$ [44]. It is larger than $M_{Z(3930)}-M_{X(3915)}$, and therefore does not support the assignment of the $X(3915)$ and $Z(3930)$ as the $\chi_{c 0}^{\prime}$ and $\chi_{c 2}^{\prime}$ simultaneously. Based on the above arguments, we shall let the mass of the $\chi_{c 0}^{\prime}$ run in a range from $3800 \mathrm{MeV}$ to $3930 \mathrm{MeV}$ which covers the predicted values from quark models [77, 50].

In the observed spectrum of the charmonia, the only candidate of the $\chi_{c 1}^{\prime}$ with wellestablished quantum numbers is the $X(3872)$ discovered by the Belle Collaboration 78 . However, due to the proximity to the $D \bar{D}^{*}$ threshold, the interpretation of the $X(3872)$ as a molecular state [79, 80] or virtual state [81] (for an update of the latter analysis see Ref. [82]) is very intriguing (for reviews, see, e.g. Refs. [5, 6, 7, 8]). Therefore, although a molecular interpretation of the $X(3872)$ was questioned in Ref. [83] (see also Ref. [84 for a critical re-evaluation), we shall not identify the $X(3872)$ as the $\chi_{c 1}^{\prime}$, and the mass of the $\chi_{c 1}^{\prime}$ will also be allowed to vary. To be specific, a range from $3.83 \mathrm{GeV}$ to $3.93 \mathrm{GeV}$ will be chosen which covers the the predicted value from some quark models, e.g. Ref. [50], and the mass of the $X(3872)$.

\subsection{Transitions between the $S$-wave charmonia}

The decays studied in this class are $\psi^{\prime} \rightarrow J / \psi \pi^{0}$ and $\psi^{\prime} \rightarrow J / \psi \eta$. As shown in Sec. 3.4 (see especially Table 4), for these transitions the charmed meson loops are enhanced by $1 / v$. The velocity can be estimated as $v \sim \sqrt{\left(2 M_{\hat{D}}-M_{\hat{\psi}}\right) / M_{\hat{D}}} \simeq 0.53$ with $M_{\hat{D}}$ being the averaged charmed-meson mass, and $M_{\hat{\psi}}=\left(M_{J / \psi}+M_{\psi^{\prime}}\right) / 2$. Hence, the LO result for the width is provided by the loops [32], although the relatively large expansion parameter leads to a sizable uncertainty.

Taking into account only the loop contributions, we give the numerical results for the $\psi^{\prime} \rightarrow J / \psi \pi^{0}$ and $\psi^{\prime} \rightarrow J / \psi \eta$. To account for the non-relativistic normalization, one needs to multiply the amplitudes by a proper factor.

One way to do this is to multiply the amplitude for each loop by the factor $\sqrt{M_{\psi^{\prime}} M_{J / \psi}} \times$ $m_{1} m_{2} m_{3}$, with $m_{i}$ being the masses of the charmed mesons in the loop. In this way, the factor may differ for different loops. From the decay $D^{*} \rightarrow D \pi$ the coupling $g$, defined in Eq. (27), can be fixed to $g=0.6$ using $F=92.4 \mathrm{MeV}$. We thus get for the decay widths

$$
\begin{aligned}
\Gamma\left(\psi^{\prime} \rightarrow J / \psi \pi^{0}\right) & =(0.048 \pm 0.025) g_{2}^{2}{g_{2}^{\prime}}^{2} \mathrm{keV}, \\
\Gamma\left(\psi^{\prime} \rightarrow J / \psi \eta\right) & =(0.43 \pm 0.23) g_{2}^{2}{g_{2}^{\prime}}^{2} \mathrm{keV},
\end{aligned}
$$

where an uncertainty of $53 \%$, which is the value of $v$, has been taken into account. Here and in the following values for $g_{2}$ and $g_{2}^{\prime}$ are given in unit of $\mathrm{GeV}^{-3 / 2}$. The resulting ratio, which is parameter-free, reads

$$
R_{\pi^{0} / \eta}=0.11 \pm 0.06
$$

with an uncertainty of $53 \%$. Comparing with the measured values listed in Table 1, it is within two sigma of the CLEO and PDG-fit data, and even overlaps within uncertainties with the result given by the BES Collaboration. A more conservative estimate of the uncertainty of the ratio may be given by assuming the uncertainties in Eq. (54) are uncorrelated, which would give $R_{\pi^{0} / \eta}$ in a much larger range from 0.03 to 0.36 . It is consistent with the data in Table 1. 
Within the effective field theory we can not predict the absolute rates for the decays, for the couplings are unknown. However, we may use the results given above to extract some averaged coupling constants that may then be compared to the corresponding values in the literature. Using the experimental data for two decay widths $\Gamma\left(\psi^{\prime} \rightarrow J / \psi \pi^{0}\right)=0.40 \pm 0.03 \mathrm{keV}$ and $\Gamma\left(\psi^{\prime} \rightarrow J / \psi \eta\right)=10.0 \pm 0.4 \mathrm{keV}$ [44], the value of $G \equiv \sqrt{g_{2} g_{2}^{\prime}}$ can be deduced. Since with the more conservative uncertainty estimate a ratio consistent with the data is obtained, a combined fit to both widths is possible. The widths for both decays agree with the data within uncertainties giving $G \approx 2.0 \mathrm{GeV}^{-3 / 2}$. We can define dimensionless coupling constants $g_{\psi D_{(s)}^{(*)} D_{(s)}^{(*)}}=g_{2} \sqrt{M_{J / \psi} M_{D_{(s)}^{(*)}} M_{D_{(s)}^{(*)}}}$ and similar quantities for the $\psi^{\prime}$. Assuming the coupling constants are $\mathrm{SU}(6)$ symmetric, i.e. $g_{\psi D D}=g_{2} \sqrt{M_{J / \psi} \bar{m}^{2}}$ with $\bar{m}$ being the average mass of the charmed meson spin-flavor SU(6) multiplet, we obtain $G_{\psi D D} \equiv \sqrt{g_{\psi D D} g_{\psi^{\prime} D D}} \approx 7.3$.

Another way to account for the non-relativistic normalization is to use an overall normalization factor, i.e., $\sqrt{M_{\psi^{\prime}} M_{J / \psi}} \bar{m}^{3}$. In this way, the results are collected as follows

$$
\begin{aligned}
\Gamma\left(\psi^{\prime} \rightarrow J / \psi \pi^{0}\right) & =(0.098 \pm 0.052) g_{2}^{2}{g_{2}^{\prime}}^{2} \mathrm{keV}, \\
\Gamma\left(\psi^{\prime} \rightarrow J / \psi \eta\right) & =(0.84 \pm 0.45) g_{2}^{2}{g_{2}^{\prime}}^{2} \mathrm{keV},
\end{aligned}
$$

and their ratio is almost the same as that given in Eq. (55). The combined coupling constant is extracted from a combined fit as $G \approx 1.7 \mathrm{GeV}^{-3 / 2}$ and the dimensionless one is $G_{\psi D D} \approx 6.0$.

Note that because the $\psi^{\prime}$ and $J / \psi$ are below the open charm threshold, their coupling to the charmed mesons cannot be extracted directly using the decay widths. Nevertheless, there are several theoretical estimates. Using vector meson dominance, the value for the $J / \psi D D$ coupling was estimated to be 7.7 in Ref. [85] and 8.0 \pm 0.5 in Ref. [86]. Using QCD sum rules, Ref. [87] obtained $8.2 \pm 1.3$, and using an SU(4) chiral model, it was estimated to be 4.93 in Ref. 88. Assuming $g_{\psi D D}=g_{\psi^{\prime} D D}$, the $J / \psi D D$ coupling extracted from the $\psi^{\prime} \rightarrow J / \psi \pi^{0}(\eta)$ considering only the charmed meson loops is of similar size as these phenomenological estimates.

Since the results for the widths considering these two ways to account for the nonrelativistic normalization are consistent within uncertainties, in the following, we shall only use the former one, i.e. using the physical masses of the intermediate mesons in the factor $\sqrt{M_{i} M_{f}} m_{1} m_{2} m_{3}$.

\subsection{Transitions between the $S$ - and $P$-wave charmonia}

In these transitions, the situation is different. As analyzed in Section 3.4, the loops do not necessarily dominate the transitions. Especially for those decays which have a very small phase space, the contribution from the charmed-meson loops is highly suppressed [48]. For these decays the momentum of the emitted pion is so small, that $q^{2} /\left(v^{3} M_{D}^{2}\right) \ll 1$. Two examples are given by $\psi^{\prime} \rightarrow h_{c} \pi^{0}$, with $q=86 \mathrm{MeV}$, and $\eta_{c}^{\prime} \rightarrow \chi_{c 0} \pi^{0}$, with $q=171 \mathrm{MeV}$. Considering that the velocity $v$ is about $\sqrt{\left[2 M_{\hat{D}}-\left(M_{\psi^{\prime}}+M_{h_{c}}\right) / 2\right] / M_{\hat{D}}} \simeq 0.4$, in case of the former transition the dimensionless factor is

$$
\frac{1}{v^{3}} \frac{\vec{q}^{2}}{m_{D}^{2}} \approx 0.03 .
$$

Thus, if all couplings are natural, the heavy-meson loop contributions are estimated to only give a few percent correction to the tree-level contribution. In case only loops are considered 
we get for the width of the $\psi^{\prime} \rightarrow h_{c} \pi^{0}$ the very small prediction,

$$
\Gamma\left(\psi^{\prime} \rightarrow h_{c} \pi^{0}\right)_{\text {loop }}=2.1 \times 10^{-7} g_{1}^{2} g_{2}^{\prime 2} \mathrm{keV},
$$

where the value of $g_{1}$ is given in unit of $\mathrm{GeV}^{-1 / 2}$. The decay width has been measured by the BES-III Collaboration very recently [89], and the absolute value of the branching ratio for the $\psi^{\prime} \rightarrow h_{c} \pi^{0}$ is $\mathcal{B}\left(\psi^{\prime} \rightarrow \pi^{0} h_{c}\right)=(8.4 \pm 1.3 \pm 1.0) \times 10^{-4}$. Using the PDG value for the width of the $\psi^{\prime}, \Gamma\left(\psi^{\prime}\right)=304 \pm 9 \mathrm{keV}$ [44], the measured width is $\Gamma\left(\psi^{\prime} \rightarrow h_{c} \pi^{0}\right)=0.26 \pm 0.05 \mathrm{keV}$. Taking into account that $g_{2}^{\prime}$ is about $2 \mathrm{GeV}^{-3 / 2}$ as extracted in Section 4.1] and Ref. [32], and $g_{1}$ is about $-4 \mathrm{GeV}^{1 / 2}$ from an estimate using the vector meson dominance [70], the numerical value $\Gamma\left(\psi^{\prime} \rightarrow h_{c} \pi^{0}\right)_{\text {loop }} \sim 10^{-5} \mathrm{keV}$ is orders of magnitude smaller than the measured value. This confirms our very rough estimate for the loop contributions in the above.

The same situation happens to the $\eta_{c}^{\prime} \rightarrow \chi_{c 0} \pi^{0}$. For this decay, the momentum of the pion is $171 \mathrm{MeV}$, and the suppression factor is

$$
\frac{1}{v^{3}} \frac{\vec{q}^{2}}{m_{D}^{2}} \approx 0.1 .
$$

Thus, here, for natural couplings, the loops are expected to give a correction of the order of $10 \%$ to the tree-level amplitude. If again only loops are considered, the width is again quite small

$$
\Gamma\left(\eta_{c}^{\prime} \rightarrow \chi_{c 0} \pi^{0}\right)_{\text {loop }}=1.0 \times 10^{-5} g_{1}^{2} g_{2}^{\prime 2} \mathrm{keV} .
$$

The pion momentum is $382 \mathrm{MeV}$ for the transitions $h_{c} \rightarrow J / \psi \pi^{0}$, and $387 \mathrm{MeV}$ for the $\chi_{c 0} \rightarrow \eta_{c} \pi^{0}$. The dimensionless suppression factor is about 0.3 , still a small number although much larger than the previous two cases. Especially through the interference with the treelevel amplitude meson loops may give a significant contribution for the mentioned decays and should not be neglected in any quantitative analysis. The widths, considering only the loop contributions, are given by

$$
\begin{aligned}
\Gamma\left(h_{c} \rightarrow J / \psi \pi^{0}\right)_{\text {loop }} & =1.9 \times 10^{-4} g_{1}^{2} g_{2}^{2} \mathrm{keV}, \\
\Gamma\left(\chi_{c 0} \rightarrow \eta_{c} \pi^{0}\right)_{\text {loop }} & =3.3 \times 10^{-4} g_{1}^{2} g_{2}^{2} \mathrm{keV} .
\end{aligned}
$$

For the transitions $\chi_{c 0}^{\prime} \rightarrow \eta_{c}^{\prime} \pi^{0}$ and $h_{c}^{\prime} \rightarrow \psi^{\prime} \pi^{0}$, the situation is more complicated. Although the pion momentum is small for these decays, the dimensionless factor $\left(q_{\pi} / M_{D}\right)^{2} / v^{3}$ does not necessarily gives a large suppression. For these two transitions, the masses of both the initial and final charmonia are close to the thresholds of the charmed mesons involved in the loops. As a result, the velocity as approximated by $\sqrt{|\bar{b}| / \bar{M}_{D}}$, where $|\bar{b}|=\left(\left|b_{12}\right|+\left|b_{23}\right|\right) / 2$, is small. As discussed at the beginning of this section, the $\chi_{c 0}^{\prime}$ and $h_{c}^{\prime}$ have not been unambiguously identified so far. To illustrate the point, one may use values for their masses from quark model calculations. For the $\chi_{c 0}^{\prime} \rightarrow \eta_{c}^{\prime} \pi^{0}$, considering the loop $\left[D, \bar{D}, D^{*}\right]$, the velocity is about 0.30 if we use $M_{\chi_{c 0}^{\prime}}=3842 \mathrm{MeV}$ predicted in Ref. [50], and $\left(q_{\pi} / M_{D}\right)^{2} / v^{3} \approx 0.23$ is still a suppression factor. If we use $M_{\chi_{c 0}^{\prime}}=3916 \mathrm{MeV}$ predicted in the quark model [77], the velocity is about 0.33 , and $\left(q_{\pi} / M_{D}\right)^{2} / v^{3} \approx 0.43$. The loops are not highly suppressed, and they may have a contribution comparable to or at least not much smaller than the tree-level one. Taking into account only the loops, one gets

$$
\Gamma\left(\chi_{c 0}^{\prime} \rightarrow \eta_{c}^{\prime} \pi^{0}\right)_{\text {loop }}=0.002(0.010) g_{1}^{\prime 2} g_{2}^{\prime 2} \mathrm{keV},
$$



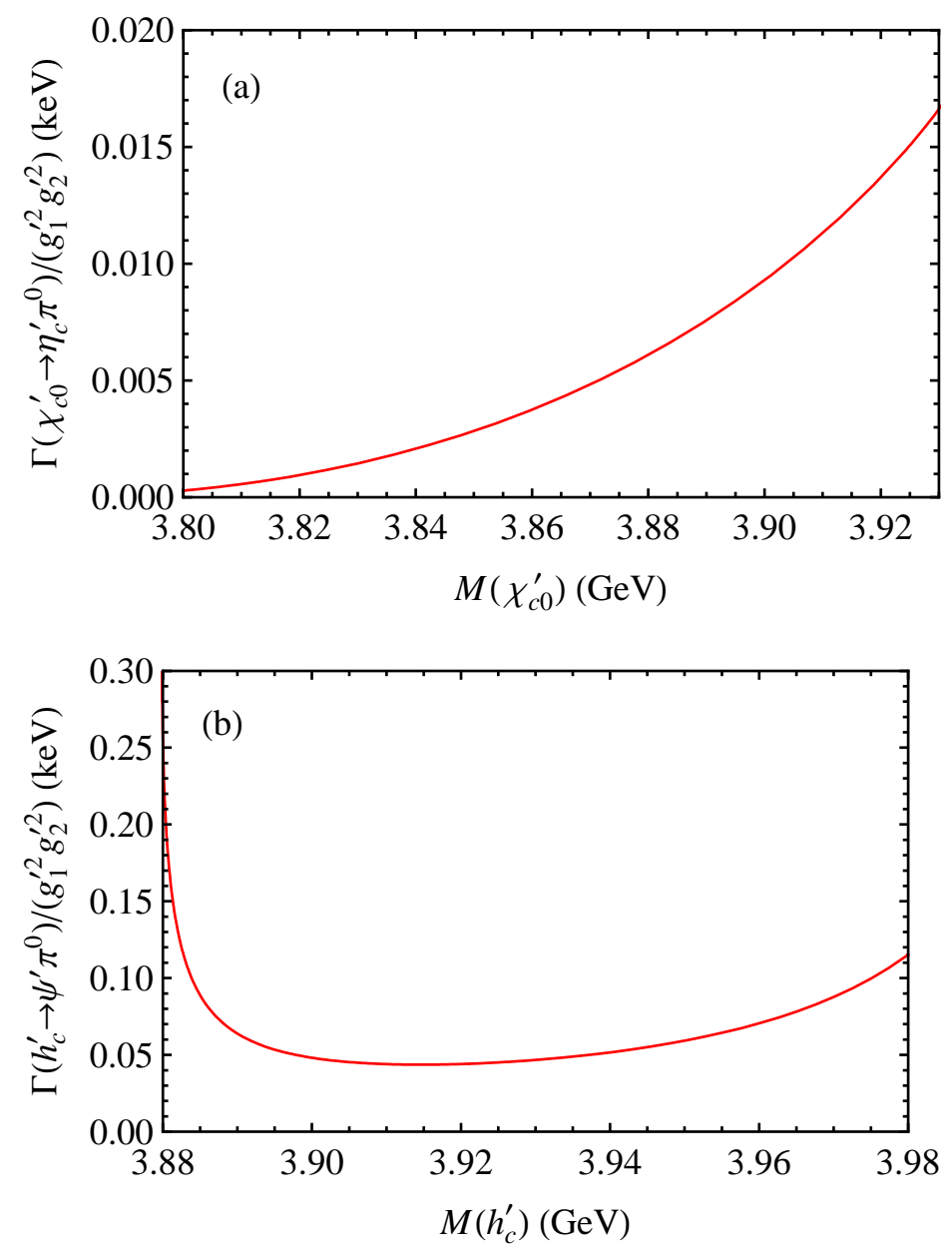

Figure 4: The widths of the decays $\chi_{c 0}^{\prime} \rightarrow \eta_{c}^{\prime} \pi^{0}$ and $h_{c}^{\prime} \rightarrow \psi^{\prime} \pi^{0}$ considering only meson loops.

where the numbers outside and inside the parentheses are obtained using $M_{\chi_{c 0}^{\prime}}$ predicted in Ref. [50] and [77], respectively - the difference reflects the difference in the factor $\left(q_{\pi} / M_{D}\right)^{2} / v^{3}$ which enters squared in the expression for the width. In Fig. 4 (a), the width of the decay $\chi_{c 0}^{\prime} \rightarrow \eta_{c}^{\prime} \pi^{0}$ as a function of $M_{\chi_{c 0}^{\prime}}$ is shown. For the decay $h_{c}^{\prime} \rightarrow \psi^{\prime} \pi^{0}$, considering the loop $\left[D^{*}, \bar{D}, D\right]$, the velocity is about 0.14 using $M_{h_{c}^{\prime}}=3908 \mathrm{MeV}$ given in Ref. [50]. As a result, the factor $\left(q_{\pi} / M_{D}\right)^{2} / v^{3} \approx 2.9$ even gives an enhancement. Then the width in this case is induced mainly by the charmed meson loops. The result for a varying $M_{h_{c}^{\prime}}$ is shown in Fig. 4 (b). In the figure, the thresholds of the $D \bar{D}$ and $D^{*} \bar{D}^{*}$ are approached at the lower and higher end, respectively. Consequently, the curve for the width shows an increasing tendency at both ends. Because the width of the $\chi_{c 0}^{\prime} \rightarrow \eta_{c}^{\prime} \pi^{0}$ is tree-level dominated, and that of the $h_{c}^{\prime} \rightarrow \psi^{\prime} \pi^{0}$ is dominated by loops, which give an enhancement, we predict their ratio to be significantly smaller than the one derived from the assumption that both are tree-level dominated

$$
\frac{\Gamma\left(\chi_{c 0}^{\prime} \rightarrow \eta_{c}^{\prime} \pi^{0}\right)_{\text {tree }}}{\Gamma\left(h_{c}^{\prime} \rightarrow \psi^{\prime} \pi^{0}\right)_{\text {tree }}}=3 \frac{q_{1}}{q_{2}} \frac{M_{\eta_{c}^{\prime}} M_{h_{c}^{\prime}}}{M_{\chi_{c 0}^{\prime}} M_{\psi^{\prime}}},
$$




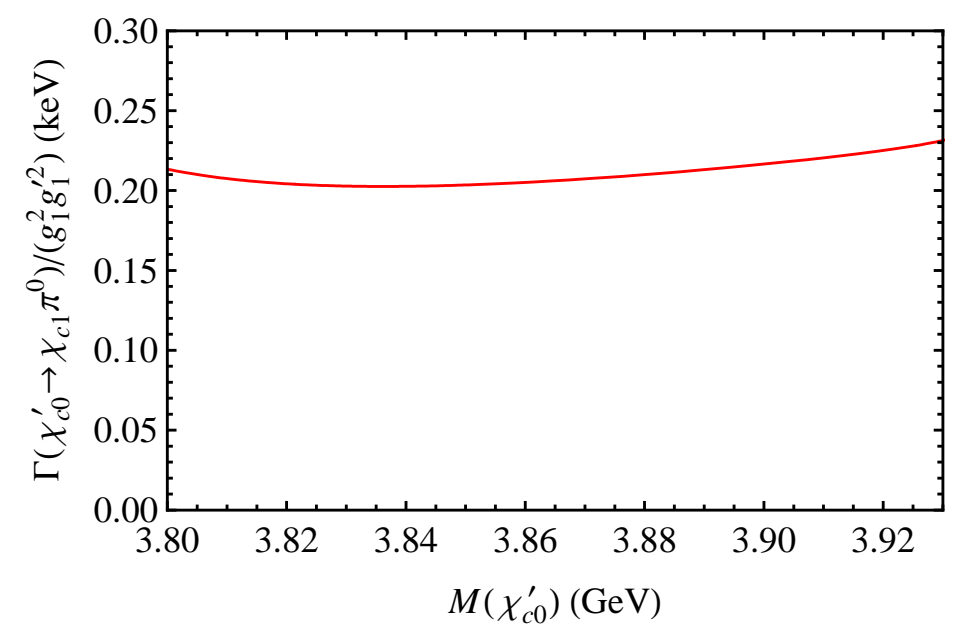

Figure 5: The width of the decay $\chi_{c 0}^{\prime} \rightarrow \chi_{c 1} \pi^{0}$.

where $q_{1}$ and $q_{2}$ are the pion momenta in the rest frame of the initial state for the decays $\chi_{c 0}^{\prime} \rightarrow \eta_{c}^{\prime} \pi^{0}$ and $h_{c}^{\prime} \rightarrow \psi^{\prime} \pi^{0}$, respectively. Once the masses of the $\chi_{c 0}^{\prime}$ and $h_{c}^{\prime}$ are measured, our statement on the ratio can be tested.

\subsection{Transitions between two $P$-wave charmonia}

As has been shown in Section 3.4, the charmed meson loops scale as $q \Delta / v^{3}$. Compared to the tree-level amplitudes, the loops are enhanced by a factor of $1 / v^{3}$, see Table 2 ,

Therefore, it is reasonable to neglect the contributions from the tree-level diagrams for the transitions between two $P$-wave charmonia. There are in total seven transition processes from the first radial excited $P$-wave charmonia to the ground state ones. All of the amplitudes are proportional to the product of the same coupling constants $g g_{1} g_{1}^{\prime} / F$. Therefore, the ratios among these decay widths can be predicted without any free parameter.

Instead of taking some value of the unknown coupling constants $g_{1}$ and $g_{1}^{\prime}$ from model estimates, we choose to show the coupling-constant dependent width for the $\chi_{c 0}^{\prime} \rightarrow \chi_{c 1} \pi^{0}$ in Fig. 5. The curve is obtained by setting the coupling constants $g_{1}$ and $g_{1}^{\prime}$ to $1 \mathrm{GeV}^{-1 / 2}$, and the width can be obtained by multiplying the value plotted in the figure by $g_{1}^{2} g_{1}^{\prime 2}$.

The mass of the $\chi_{c 1}^{\prime}$ is also allowed to vary, and the width for the $\chi_{c 1}^{\prime} \rightarrow \chi_{c 0} \pi^{0}$ is shown in Fig. 6 (a). In Fig. [6 (b), the parameter-free ratio of the decay widths $\Gamma\left(\chi_{c 1}^{\prime} \rightarrow\right.$ $\left.\chi_{c 0} \pi^{0}\right)_{\text {loop }} / \Gamma\left(\chi_{c 0}^{\prime} \rightarrow \chi_{c 1} \pi^{0}\right)_{\text {loop }}$ is shown. In the plots, a double-cusp structure is prominent. These cusps correspond to the thresholds of the neutral and charged $D \bar{D}^{*}$ mesons. When the thresholds are approaching, the velocities of the intermediate charmed mesons decrease, and then the enhancement characterized by $1 / v^{3}$ becomes larger. A similar double-cusp structure was found in a study of $a_{0}(980)-f_{0}(980)$ mixing in the decays of charmonia $23,24,90$, where the cusps are due to the thresholds of the charged and neutral kaons 91. For the decays of the $\chi_{c 1}^{\prime} \rightarrow \chi_{c J} \pi^{0}$, the ratios defined as

$$
R_{1012}^{\text {loop }} \equiv \frac{\Gamma\left(\chi_{c 1}^{\prime} \rightarrow \chi_{c 0} \pi^{0}\right)_{\text {loop }}}{\Gamma\left(\chi_{c 1}^{\prime} \rightarrow \chi_{c 2} \pi^{0}\right)_{\text {loop }}}, \quad R_{1112}^{\text {loop }} \equiv \frac{\Gamma\left(\chi_{c 1}^{\prime} \rightarrow \chi_{c 1} \pi^{0}\right)_{\text {loop }}}{\Gamma\left(\chi_{c 1}^{\prime} \rightarrow \chi_{c 2} \pi^{0}\right)_{\text {loop }}}
$$



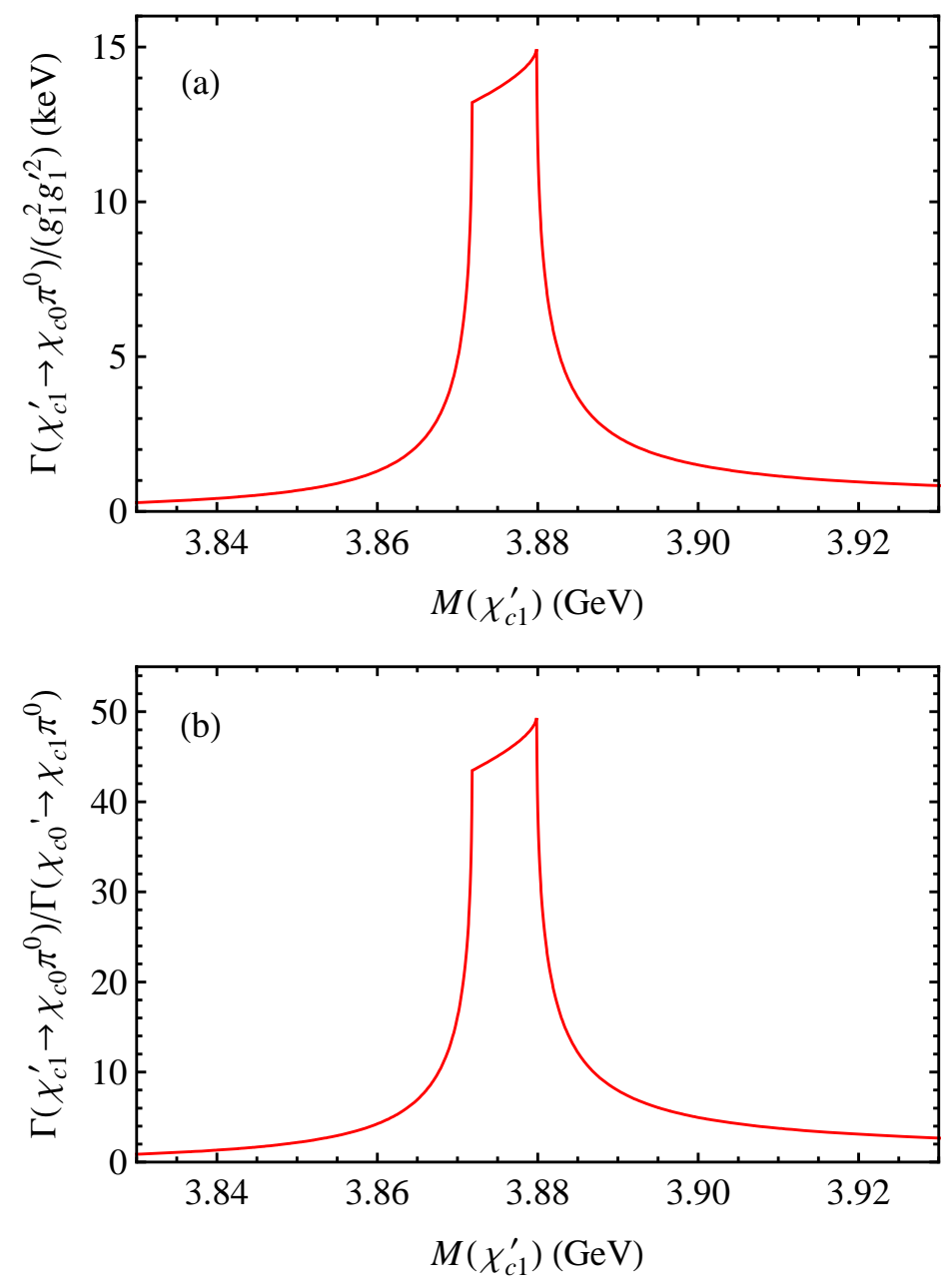

Figure 6: (a) The width of the decay $\chi_{c 1}^{\prime} \rightarrow \chi_{c 0} \pi^{0}$. (b) The parameter-free ratio between the two decay widths $\Gamma\left(\chi_{c 1}^{\prime} \rightarrow \chi_{c 0} \pi^{0}\right)_{\text {loop }} / \Gamma\left(\chi_{c 0}^{\prime} \rightarrow \chi_{c 1} \pi^{0}\right)_{\text {loop }}$, here $M_{\chi_{c 0}^{\prime}}=3842 \mathrm{MeV}$ as predicted in Ref. [50] is used.

are plotted in Fig. 7 as the solid and dashed lines, respectively, in a parameter-free way. One may also construct similar ratios taking into account only the tree-level contributions, which are

$$
R_{1012}^{\mathrm{tree}}=\frac{4 q_{\pi 0}^{3}}{5 q_{\pi 2}^{3}} \frac{M_{\chi_{c 0}}}{M_{\chi_{c 2}}}, \quad R_{1112}^{\mathrm{tree}}=\frac{3 q_{\pi 1}^{3}}{5 q_{\pi 2}^{3}} \frac{M_{\chi_{c 1}}}{M_{\chi_{c 2}}}
$$

where $q_{\pi J}$ is the pion momentum for the decays $\chi_{c 1}^{\prime} \rightarrow \chi_{c J} \pi^{0}$. The dotted and dot-dashed curves in Fig. 7 represent these ratios, respectively. Comparing the curves considering only the meson loops with the tree-level ones, the difference is not tremendous. Since the uncertainty is large in our loop calculations, c.f. Eq. (55) and the discussion below, it may be difficult to draw a definite conclusion from the comparison without a refined uncertainty analysis.

The ratios for the tree-level contributions agree with those derived in Ref. [58] using chiral Lagrangians, but differ from those given for the $c \bar{c}$ option of the $X(3872)$ in Ref. [92] using 


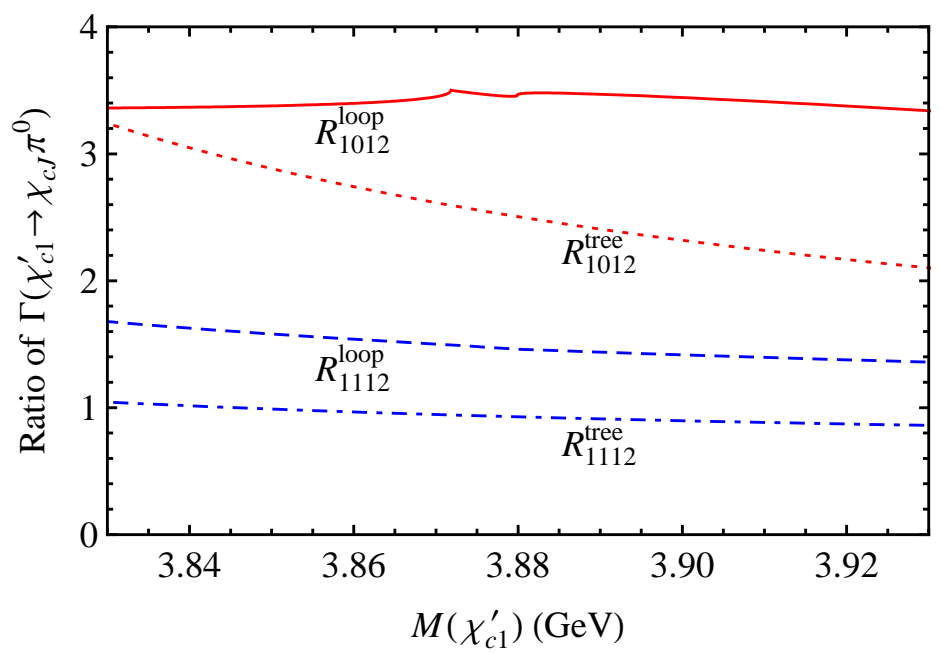

Figure 7: The parameter-free ratios among the decay widths of the $\chi_{c 1}^{\prime} \rightarrow \chi_{c J} \pi^{0}$ considering only the meson loops. The corresponding tree-level predictions are also shown.

the QCDME. However, as shown above, even if the $X(3872)$ is a $c \bar{c}$ state, its decays into the $\chi_{c J} \pi^{0}$ are mainly given by the intermediate charmed meson loops, i.e. by non-mulitpole contributions.

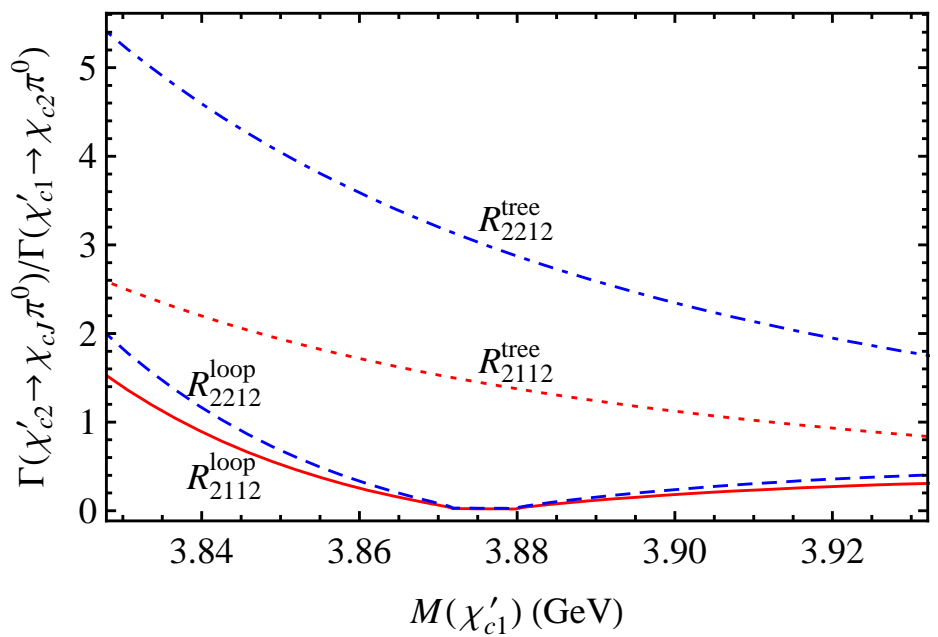

Figure 8: Ratio of the decay widths $\chi_{c 2}^{\prime} \rightarrow \chi_{c 1} \pi^{0}$ and $\chi_{c 2}^{\prime} \rightarrow \chi_{c 2} \pi^{0}$ to $\Gamma\left(\chi_{c 1}^{\prime} \rightarrow \chi_{c 2} \pi^{0}\right)$.

The widths of the $\chi_{c 2}^{\prime}$ decays into the $\chi_{c 1} \pi^{0}$ and $\chi_{c 2} \pi^{0}$ considering only the meson loops are

$$
\begin{aligned}
& \Gamma\left(\chi_{c 2}^{\prime} \rightarrow \chi_{c 1} \pi^{0}\right)_{\text {loop }}=(0.08 \pm 0.03) g_{1}^{2} g_{1}^{\prime 2} \mathrm{keV} \\
& \Gamma\left(\chi_{c 2}^{\prime} \rightarrow \chi_{c 2} \pi^{0}\right)_{\text {loop }}=(0.10 \pm 0.04) g_{1}^{2} g_{1}^{\prime 2} \mathrm{keV}
\end{aligned}
$$

where the uncertainties are $33 \%$ and $36 \%$, which are the velocities of the intermediate charmed 


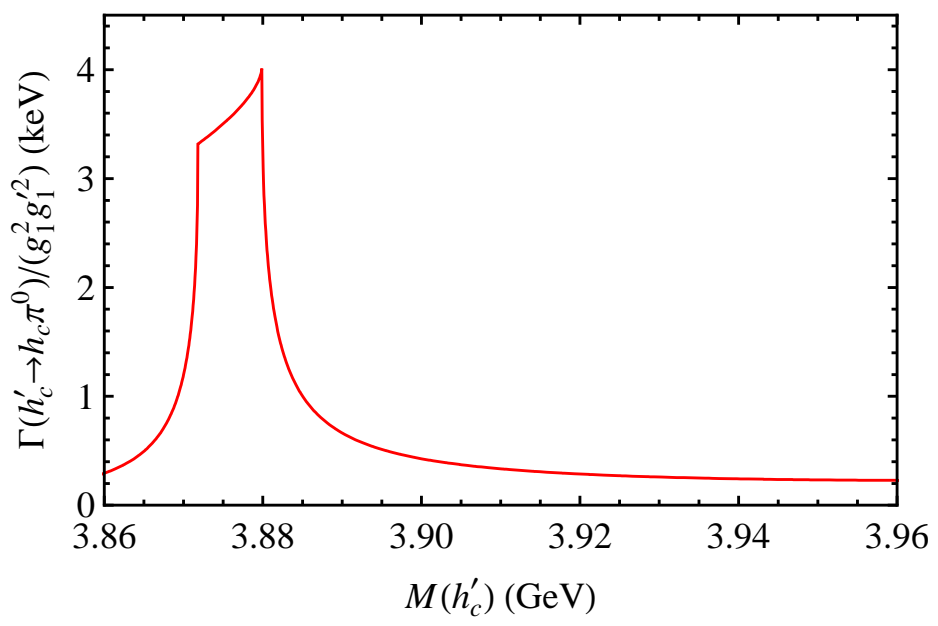

Figure 9: The width of the decay $h_{c}^{\prime} \rightarrow h_{c} \pi^{0}$ considering only the meson loops.

mesons for these two processes, respectively. The ratios of decay widths defined as

$$
R_{2112}^{\text {loop }} \equiv \frac{\Gamma\left(\chi_{c 2}^{\prime} \rightarrow \chi_{c 1} \pi^{0}\right)_{\text {loop }}}{\Gamma\left(\chi_{c 1}^{\prime} \rightarrow \chi_{c 2} \pi^{0}\right)_{\text {loop }}}, \quad R_{2212}^{\text {loop }} \equiv \frac{\Gamma\left(\chi_{c 2}^{\prime} \rightarrow \chi_{c 2} \pi^{0}\right)_{\text {loop }}}{\Gamma\left(\chi_{c 1}^{\prime} \rightarrow \chi_{c 2} \pi^{0}\right)_{\text {loop }}}
$$

for the loop contributions only are shown as the solid and dashed lines in Fig. 8. The same ratios considering only the tree-level contributions

$$
R_{2112}^{\text {tree }}=\frac{3 q_{1}^{3}}{5 q_{2}^{3}} \frac{M_{\chi_{c 1}} M_{\chi_{c 1}^{\prime}}}{M_{\chi_{c 2}^{\prime}} M_{\chi_{c 2}}}, \quad R_{2212}^{\text {tree }}=\frac{9 q_{1}^{3}}{5 q_{2}^{3}} \frac{M_{\chi_{c 1}^{\prime}}}{M_{\chi_{c 2}^{\prime}}}
$$

where $q_{i}$ is the pion momentum for the corresponding decay, are given by the dotted and dotdashed lines in Fig. 8, respectively. The difference is obvious, and may be tested by future experiments. The width of the decay $h_{c}^{\prime} \rightarrow h_{c} \pi^{0}$ considering only the meson loops is shown in Fig. 9. The double-cusp structure is again due to the coupling to the intermediate $D \bar{D}^{*}$ mesons.

\section{Comparison with the effective Lagrangian approach}

As emphasized before, the power counting of NREFT provides a control of the theoretical uncertainties. However, for transitions where the mass difference between the initial and final charmonium exceeds $\sim 800 \mathrm{MeV}$, i.e. $\mathcal{O}\left(\Lambda_{\chi}\right)$, the NREFT is not applicable any more. The ELA, on the other hand, which deals with the non-local effects by introducing empirical form factors to cut off the divergences, can be applied to broader kinematic regions. But the disadvantage is that, due to a lack of knowledge about the behavior of the counterterms, model-dependence will be present in association with the cut-off energies and different forms for the form factors. By comparing these two approaches with each other, we expect that the model-dependent aspects of the ELA can be identified and reduced. In this way, one may have more confidence in the ELA when it is applied in kinematical regions where the NREFT is not applicable. 
In this section, we first present the general formulae for the ELA. The calculation results will be summarized and compared with the NREFT for those transitions studied in the previous section. The detailed formulation is given in Appendix C.

The loop transition amplitudes for the transitions in Fig. 2 can be expressed in a general form in the ELA as follows:

$$
M_{f i}=\int \frac{d^{4} q_{2}}{(2 \pi)^{4}} \sum_{D^{*} \text { pol. }} \frac{V_{1} V_{2} V_{3}}{a_{1} a_{2} a_{3}} \prod_{i} \mathcal{F}_{i}\left(m_{i}, q_{i}^{2}\right)
$$

where $V_{i}(i=1,2,3)$ are the vertex functions; $a_{i}=q_{i}^{2}-m_{i}^{2}(i=1,2,3)$ are the denominators of the intermediate meson propagators. We adopt the form factor, $\prod_{i} \mathcal{F}_{i}\left(m_{i}, q_{i}^{2}\right)$, which is a product of monopole form factors for each internal meson, i.e.

$$
\prod_{i} \mathcal{F}_{i}\left(m_{i}, q_{i}^{2}\right) \equiv \mathcal{F}_{1}\left(m_{1}, q_{1}^{2}\right) \mathcal{F}_{2}\left(m_{2}, q_{2}^{2}\right) \mathcal{F}_{3}\left(m_{3}, q_{3}^{2}\right)
$$

with

$$
\mathcal{F}_{i}\left(m_{i}, q_{i}^{2}\right) \equiv\left(\frac{\Lambda_{i}^{2}-m_{i}^{2}}{\Lambda_{i}^{2}-q_{i}^{2}}\right),
$$

where $\Lambda_{i} \equiv m_{i}+\alpha \Lambda_{\mathrm{QCD}}$ [93] and the QCD energy scale is $\Lambda_{\mathrm{QCD}}=220 \mathrm{MeV}$. The numerator is introduced in order not to modify the expression at the on-shell point. This form factor is supposed to parameterize the non-local effects of the vertex functions and to remove the loop divergence in the integrals. In this approach the local couplings for a charmonium to charmed mesons, or a light meson to charmed mesons are the same as used in the NREFT, while the form factor parameter $\alpha$ will be determined by comparison to experimental information. Thus, it is assumed here that all — or at least the dominant part — of the short-range physics related to meson loops can be parameterized in the form of Eq. (72) with a reaction independent parameter $\alpha$.

Although used widely and very convenient for the actual evaluation of the pertinent integrals, the form factor parameterization given in Eq. (71) also has its drawbacks. Especially, unphysical thresholds, which are located at $\Lambda_{i}+m_{j}=m_{i}+m_{j}+\alpha \Lambda_{\mathrm{QCD}}$ and $\Lambda_{i}+\Lambda_{j}=m_{i}+m_{j}+2 \alpha \Lambda_{\mathrm{QCD}}$, are introduced into the integrals. Thus, for sufficiently heavy decaying charmonia and small values of $\alpha$, these singularities are located nearby or even inside the physical regime and additionally introduce unphysical contributions. Clearly, those are not part of the NREFT. We therefore expect, and indeed observe, that there exist significant quantitative differences between these two approaches in the decays of heavy charmonia. It suggests that a different form factor parameterization or larger values of $\alpha$ should be considered. On the other hand, for those cases, where the form factor singularities do not contribute, we expect interesting insights from the comparison of the ELA and NREFT results.

\subsection{Transitions between the $S$-wave charmonia}

For transitions between the $S$-wave charmonia, i.e. $\psi^{\prime} \rightarrow J / \psi \pi^{0}(\eta)$, we plot the $\alpha$-dependence of the partial decay widths of $\psi^{\prime} \rightarrow J / \psi \pi^{0}(\eta)$ in Fig. 10 (a) as shown by the solid and dashed lines, respectively. The $\pi^{0}-\eta$ mixing has been taken into account. Using $\alpha$ in a range of $\alpha \simeq 1 \ldots 2$, the measured ratio $R_{\pi^{0} / \eta}$ by both the CLEO and BES Collaborations can be reproduced, and the central value of the PDG fit as listed in Table 1 may be obtained with 


\begin{tabular}{l|l|c|c}
\hline \hline & Decays & Qualitative & Quantitative \\
\hline \multirow{5}{*}{$S S$} & $\psi^{\prime} \rightarrow J / \psi \pi^{0}$ & $\sqrt{ }$ & $\sqrt{ }$ \\
& $\psi^{\prime} \rightarrow J / \psi \eta$ & $\sqrt{ }$ & $\sqrt{ }$ \\
\hline \multirow{5}{*}{$S P$} & $\psi^{\prime} \rightarrow h_{c} \pi^{0}$ & $\sqrt{ }$ & $\times$ \\
& $h_{c} \rightarrow J / \psi \pi^{0}$ & $\sqrt{ }$ & $\sqrt{ }$ \\
& $\eta_{c}^{\prime} \rightarrow \chi_{c 0} \pi^{0}$ & $\sqrt{ }$ & $\times$ \\
& $\chi_{c 0} \rightarrow \eta_{c} \pi^{0}$ & $\sqrt{ }$ & $\times$ \\
& $h_{c}^{\prime} \rightarrow \psi^{\prime} \pi^{0}$ & $\sqrt{ }$ & $\times$ \\
& $\chi_{c 0}^{\prime} \rightarrow \eta_{c}^{\prime} \pi^{0}$ & $\sqrt{ }$ & $\times$ \\
\hline \multirow{5}{*}{$P P$} & $\chi_{c 1}^{\prime} \rightarrow \chi_{c 0} \pi^{0} / \chi_{c 0}^{\prime} \rightarrow \chi_{c 1} \pi^{0}$ & $\sqrt{ }$ & $\times$ \\
& $\chi_{c 1}^{\prime} \rightarrow \chi_{c 0} \pi^{0} / \chi_{c 1}^{\prime} \rightarrow \chi_{c 2} \pi^{0}$ & $\sqrt{ }$ & $\times$ \\
& $\chi_{c 1}^{\prime} \rightarrow \chi_{c 1} \pi^{0} / \chi_{c 1}^{\prime} \rightarrow \chi_{c 2} \pi^{0}$ & $\sqrt{ }$ & $\sqrt{ }$ \\
& $\chi_{c 2}^{\prime} \rightarrow \chi_{c 1} \pi^{0} / \chi_{c 1}^{\prime} \rightarrow \chi_{c 2} \pi^{0}$ & $\sqrt{ }$ & $\sqrt{ }$ \\
& $\chi_{c 2}^{\prime} \rightarrow \chi_{c 2} \pi^{0} / \chi_{c 1}^{\prime} \rightarrow \chi_{c 2} \pi^{0}$ & $\sqrt{ }$ & $\sqrt{ }$ \\
\hline \hline
\end{tabular}

Table 5: Summary of the qualitative and quantitative features in the comparison between the ELA and NREFT results for various decay transitions. For $P P$ transitions, we take the width ratios in order to compare the ELA with the NREFT results. The appearance of the crosses can be understood, as explained in the text.

$\alpha=1.64$,

$$
\begin{aligned}
\Gamma\left(\psi^{\prime} \rightarrow J / \psi \pi^{0}\right) & =0.031 g_{2}^{2} g_{2}^{2} \mathrm{keV}, \\
\Gamma\left(\psi^{\prime} \rightarrow J / \psi \eta\right) & =0.77 g_{2}^{2} g_{2}^{\prime 2} \mathrm{keV},
\end{aligned}
$$

where the vertex couplings are taken the same as those in the NREFT. These results are consistent with the NREFT.

In Fig. 10 (b), the ratio $R_{\pi^{0} / \eta}$ calculated in the ELA is given in terms of $\alpha$. The predominant feature is that the $\alpha$-dependence of the ratios is quite stable. It is because the loop amplitudes for the transitions play a dominant role in the transition and have the same divergence structure which is regularized by the form factors. With $\alpha=1.64$, the resulting ratio $R_{\pi^{0} / \eta}^{\text {loop }}$ is consistent with the result from the NREFT [32].

\subsection{Transitions between the $S$ - and $P$-wave charmonia}

Recall that the power counting suggests suppressions on most of the transitions between the $S$ and $P$-wave charmonia via intermediate meson loops, such as, $\psi^{\prime} \rightarrow h_{c} \pi^{0}$, while the loops in the transition $h_{c}^{\prime} \rightarrow \psi^{\prime} \pi^{0}$ are enhanced. The dominance of tree-level contribution means that the physics is described by contact interactions - sometimes loosely called short-distance physics. On the contrary, in the ELA, since form factors are adopted, part of the short-range physics is already included in the loops. Therefore, one may expect large differences between 

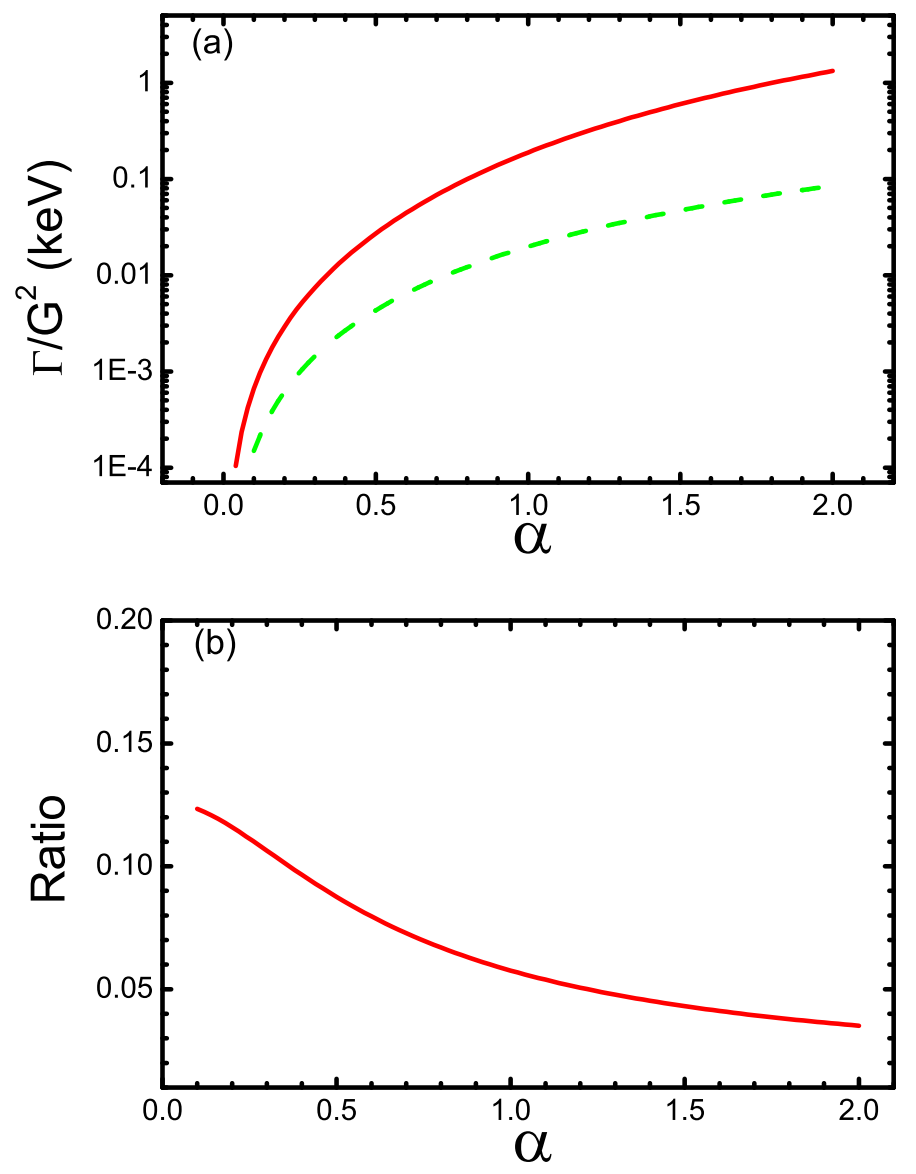

Figure 10: (a) $\alpha$-dependence for the decay widths of the decay $\psi^{\prime} \rightarrow J / \psi \pi^{0}$ (dashed line) and $J / \psi \eta$ (solid line). (b) $\alpha$-dependence of the ratio $R_{\pi^{0} / \eta}$. The coupling is defined as $G^{2} \equiv g_{2}^{2} g_{2}^{2}$.

the NREFT and the ELA in this sector, and the ELA results would typically be larger than those in the NREFT, especially for the tree-level dominant transitions.

With the form factor parameter determined in the previous subsection and the same couplings for the vertices as in the NREFT, we now examine the predictions from the ELA for those transitions. Instead of going to all the channels, we shall concentrate on two sets of decays, i) $\psi^{\prime} \rightarrow h_{c} \pi^{0}$ and $h_{c} \rightarrow J / \psi \pi^{0}$, and ii) $\eta_{c}^{\prime} \rightarrow \chi_{c 0} \pi^{0}$ and $\chi_{c 0} \rightarrow \eta_{c} \pi^{0}$, and summarize the others in Table 5 .

The two transitions in each set involve similar coupling vertices, but different kinematics. Their ratios will again eliminate the uncertainties arising from the unknown coupling constants. In Fig. 11, the decay widths with the charmonium- $D$-meson couplings normalized to unity are plotted for these two pairs of decay channels, i.e. $\psi^{\prime} \rightarrow h_{c} \pi^{0}$ (solid line), $h_{c} \rightarrow J / \psi \pi^{0}$ (dashed line), $\eta_{c}^{\prime} \rightarrow \chi_{c 0} \pi^{0}$ (dot-dashed line) and $\chi_{c 0} \rightarrow \eta_{c} \pi^{0}$ (dotted line) in a range of $\alpha=1 \ldots 2$. Note that the BES-III measurement gives a central value $\Gamma^{\exp }\left(\psi^{\prime} \rightarrow h_{c} \pi^{0}\right)=0.61 \mathrm{keV}$ [89]. Within $\alpha=1 \ldots 2$ which is found in the transitions 


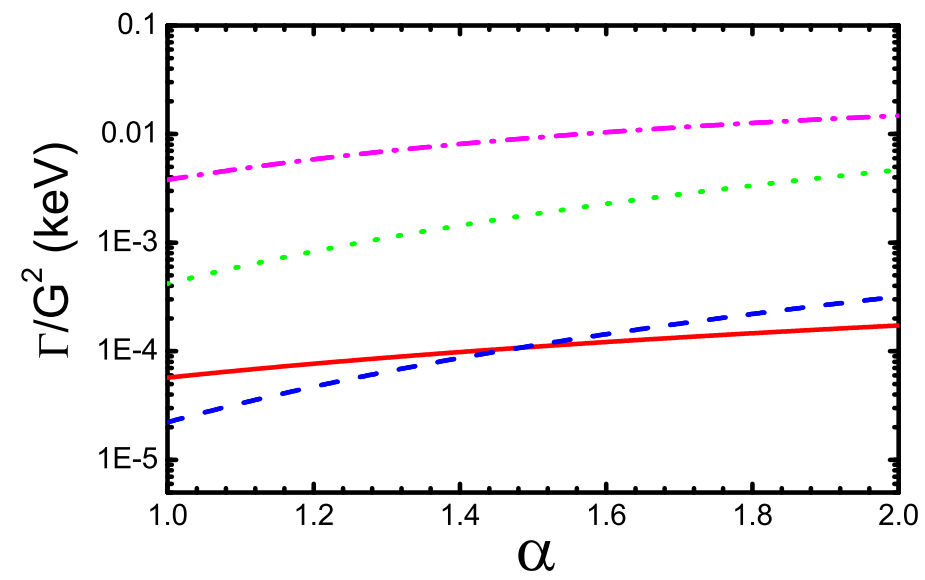

Figure 11: $\alpha$-dependence of the decay widths of $\psi^{\prime} \rightarrow h_{c} \pi^{0}$ (solid line), $h_{c} \rightarrow J / \psi \pi^{0}$ (dashed line), $\chi_{c 0} \rightarrow \eta_{c} \pi^{0}$ (dotted line), and $\eta_{c}^{\prime} \rightarrow \chi_{c 0} \pi^{0}$ (dot-dashed line). The coupling is $G^{2} \equiv g_{1}^{2} g_{2}^{\prime 2}$ for $\psi^{\prime} \rightarrow h_{c} \pi^{0}$ and $\eta_{c}^{\prime} \rightarrow \chi_{c 0} \pi^{0}$, while $G^{2} \equiv g_{1}^{2} g_{2}^{2}$ for $h_{c} \rightarrow J / \psi \pi^{0}$ and $\chi_{c 0} \rightarrow \eta_{c} \pi^{0}$.

$\psi^{\prime} \rightarrow J / \psi \pi^{0}(\eta)$, the partial decay width of $\psi^{\prime} \rightarrow h_{c} \pi^{0}$ from the meson loops is much smaller than the experimental data [89], which confirms the suppression of the meson loops found in the NREFT, although these two approaches give quite different values for the meson loop magnitudes. This comparison is listed in Table 5 as qualitative agreement.

Such a suppression also occurs to $h_{c} \rightarrow J / \psi \pi^{0}$ in the ELA, and exhibits some peculiar features in comparison with the NREFT expectation. Since the decay $h_{c} \rightarrow J / \psi \pi^{0}$ has a relatively large phase space, the power counting suppression in the NREFT is not as much as for $\psi^{\prime} \rightarrow h_{c} \pi^{0}$ as pointed out in the previous Section. In the ELA, however, as shown in Fig. 11, these two decay widths are of the same order in the range of $\alpha=1 \ldots 2$. Nevertheless, the suppression of the charmed meson loops as analyzed in the NREFT in both decays is confirmed in the ELA. The partial decay width of the transition $h_{c} \rightarrow J / \psi \pi^{0}$ considering only loops is even quantitatively consistent with the NREFT expectation.

As shown by the dotted and dot-dashed lines in Fig. 11, we also observe a suppression of the meson loops in $\eta_{c}^{\prime} \rightarrow \chi_{c 0} \pi^{0}$ and $\chi_{c 0} \rightarrow \eta_{c} \pi^{0}$ which, however, is weaker as in the NREFT calculation. As already mentioned, this may be understood as part of the short-range physics is already mimicked by the form factors in the ELA. Such an uncertainty seems inevitable in the ELA and a measurement of the ratios of branching fractions would be less sensitive to it. The normalized widths of $\eta_{c}^{\prime} \rightarrow \chi_{c 0} \pi^{0}$ and $\chi_{c 0} \rightarrow \eta_{c} \pi^{0}$ have a different ordering compared with the NREFT results, and the $\eta_{c}^{\prime} \rightarrow \chi_{c 0} \pi^{0}$ appears to be one order of magnitude larger. This is because the mass of the $\eta_{c}^{\prime}$ is much closer to the $D \bar{D}^{*}$ threshold than $\eta_{c}$, and the form factor parameterization adopted in the ELA makes the effect of the proximity more prominent than the NREFT.

The other decay channels of interest are between the radial excitation $S$ - and $P$-wave charmonia, such as $h_{c}^{\prime} \rightarrow \psi^{\prime} \pi^{0}$ and $\chi_{c 0}^{\prime} \rightarrow \eta_{c}^{\prime} \pi^{0}$. The NREFT predicts that the width of $\chi_{c 0}^{\prime} \rightarrow \eta_{c}^{\prime} \pi^{0}$ is tree-level dominant, but the charmed meson loops may give a significant contribution, while the transition $h_{c}^{\prime} \rightarrow \psi^{\prime} \pi^{0}$ is dominated by the meson loops. We find that 
the ELA result for $h_{c}^{\prime} \rightarrow \psi^{\prime} \pi^{0}$ has a much larger normalized decay width considering loops only, which indicates the dominance of the loops for this transitions. It agrees qualitatively with the NREFT one quite well though has a larger value. The ELA result of the transition $\chi_{c 0}^{\prime} \rightarrow \eta_{c}^{\prime} \pi^{0}$ is smaller than that of $h_{c}^{\prime} \rightarrow \psi^{\prime} \pi^{0}$ while much larger than that of the other $S P$ transitions. We consider this as a qualitative agreement with the NREFT, since in the NREFT the pattern is similar.

This is consistent with our prospect that these two approaches would agree with each other given the dominance of the meson loops in the transitions. It should also be pointed out that in the ELA, singularities would appear when the mass of the initial charmonium is larger than the artificial threshold $\Lambda_{1}+\Lambda_{2}$ introduced by the form factor. When the "threshold" is approaching, one typically gets larger results. The comparison of these two approaches turns out to be a useful tool for our understanding the properties of the ELA.

\subsection{Transitions between two $P$-wave charmonia}

The transitions between $P$-wave charmonia, especially for the $\chi_{c J}$ decays, allow for an especially sensitive test of the meson loop transitions, as the power counting analysis suggests that the meson loop contributions relative to the tree-level transitions will be enhanced significantly (c.f. table 4). Thus, the comparison between NREFT and ELA will help restrict the possible parameter ranges in the ELA as well as study further the implications of the adopted form factor parameterization.

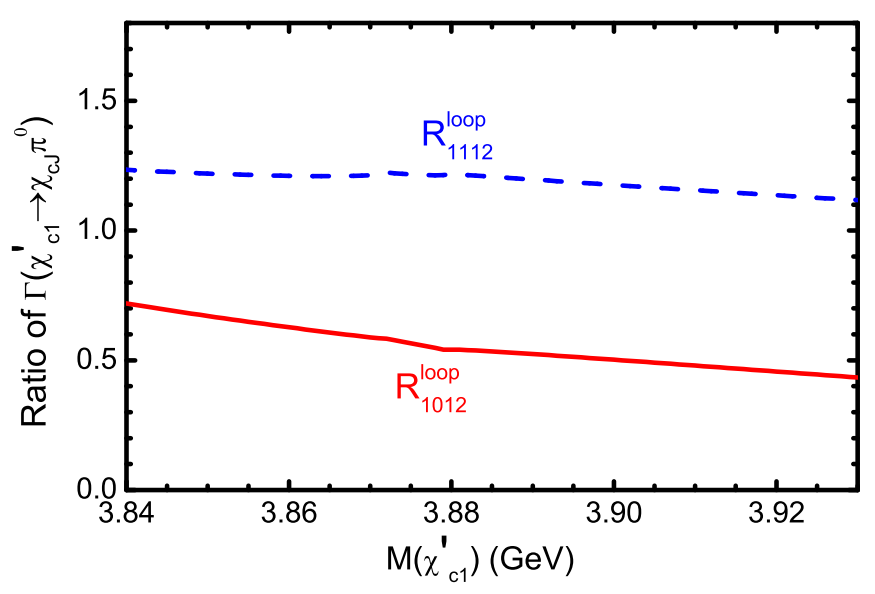

Figure 12: Parameter-free ratios among the decay widths of $\chi_{c 1}^{\prime} \rightarrow \chi_{c J} \pi^{0}$ considering only the meson loops in the ELA.

In the case of $\chi_{c 0}^{\prime} \rightarrow \chi_{c 1} \pi^{0}$ and $\chi_{c 1}^{\prime} \rightarrow \chi_{c 0} \pi^{0}$, our calculation shows that the normalized partial widths, $\Gamma\left(\chi_{c 0}^{\prime} \rightarrow \chi_{c 1} \pi^{0}\right) /\left(g_{1}^{2} g_{2}^{2}\right)$, and the mass-dependence of $\Gamma\left(\chi_{c 1}^{\prime} \rightarrow \chi_{c 0} \pi^{0}\right) /\left(g_{1}^{2} g_{1}^{\prime 2}\right)$ have similar structures as those given by the NREFT, but the relative magnitude of the decay widths is different. The ELA predicts larger results for the widths of the transitions $\chi_{c 0}^{\prime} \rightarrow \chi_{c 1} \pi^{0}$ and $\chi_{c 1}^{\prime} \rightarrow \chi_{c J} \pi^{0}$ than the corresponding values in the NREFT, except for the $\chi_{c 1}^{\prime} \rightarrow \chi_{c 0} \pi^{0}$ which is opposite. 


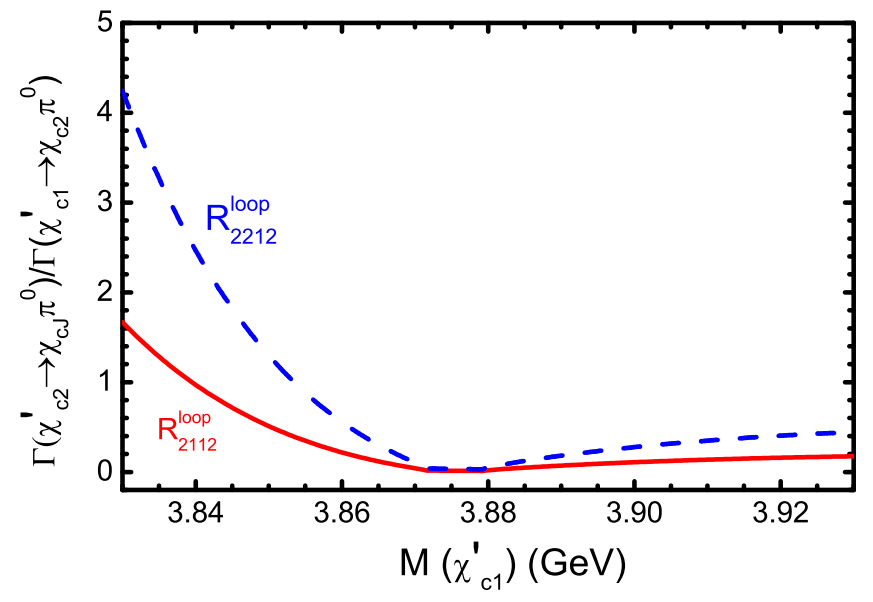

Figure 13: Ratios of the decay widths $\chi_{c 2}^{\prime} \rightarrow \chi_{c 1} \pi^{0}$ and $\chi_{c 2}^{\prime} \rightarrow \chi_{c 2} \pi^{0}$ to $\Gamma\left(\chi_{c 1}^{\prime} \rightarrow \chi_{c 2} \pi^{0}\right)$ in the ELA.

A comparison is also made for these two methods in the predictions of the ratios, $R_{1012}^{\text {loop }}$ and $R_{1112}^{\text {loop }}$, as defined by Eq. (65). In Fig. 12, these two ratios are plotted in terms of $\alpha$ values. It is sensible to observe the stability of the ratios within the varying $\chi_{c 1}^{\prime}$ mass region. In comparison with the NREFT results in Fig. (7) ratio $R_{1112}^{\text {loop }}$ is consistent with the dashed line there even in magnitude, while ratio $R_{1012}^{\text {loop }}$ exhibits an inverse relative magnitude between $\Gamma\left(\chi_{c 1}^{\prime} \rightarrow \chi_{c 0} \pi^{0}\right)$ and $\Gamma\left(\chi_{c 1}^{\prime} \rightarrow \chi_{c 2} \pi^{0}\right)$. Although there exist significant discrepancies between these two methods here, we emphasize that the flat behavior of the ratios again suggests some systematic model-independent features of the meson loop contributions. Especially, there are

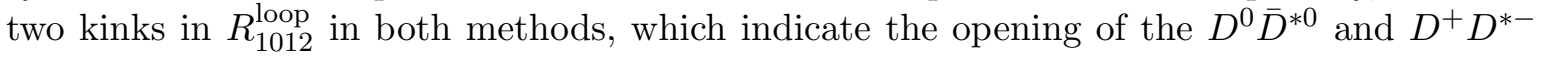
thresholds. In this sense, it is also listed as in qualitative agreement in Table 5 .

We further compare the ELA results for the $P$-wave decays, $\chi_{c 2}^{\prime} \rightarrow \chi_{c 1} \pi^{0}$ and $\chi_{c 2}^{\prime} \rightarrow \chi_{c 2} \pi^{0}$, with the NREFT ones. For these two transitions, the contributing loops have $M_{1}=M_{2}=D^{*}$. The mass of the initial state $M_{\chi_{c 2}}$ is smaller than the threshold $2 M_{D^{*}}$, and much smaller than the artificial threshold $2\left(M_{D^{*}}+\alpha \Lambda_{\mathrm{QCD}}\right)$, and hence the unphysical singularities would have little effect. So one may expect the ELA would give similar results compared with the NREFT. The meson loops in the ELA give,

$$
\begin{gathered}
\Gamma\left(\chi_{c 2}^{\prime} \rightarrow \chi_{c 1} \pi^{0}\right)_{\text {loop }}=0.06 g_{1}^{2} g_{1}^{\prime 2} \mathrm{keV}, \\
\Gamma\left(\chi_{c 2}^{\prime} \rightarrow \chi_{c 2} \pi^{0}\right)_{\text {loop }}=0.19 g_{1}^{2} g_{1}^{\prime 2} \mathrm{keV},
\end{gathered}
$$

with $\alpha=1.64$ as determined previously. They are consistent with those given by the NREFT in Eq. (67). In Fig. 13, we present the ratios of the decay widths, $R_{2112}^{\text {loop }}$ and $R_{2212}^{\text {loop }}$, as defined by Eq. (68), to compare with the solid and dashed lines in Fig. 8. It is interesting to see the consistency of these two methods here in both mass evolution and magnitude, as expected.

\subsection{Summary of the ELA results}

As can be seen from Table 5, the NREFT and ELA results for all the transitions are in qualitative agreement. For both the $S S$ and $P P$ transitions the results for the two approaches 
are in quantitative agreement as long as the decaying charmonium state is not sufficiently heavy to allow the form factor singularities to matter numerically. The observed level of agreement between these two approaches provides a further support for the power counting of the NREFT. It demonstrates nicely two important aspects of this effective field theory, namely, the applicability of the non-relativistic treatment in these charmonia transitions (note that the ELA formulations are relativistic), and the dominance of the long-range pieces of the meson loops which is driven by the unitary cuts for the $S S$ and $P P$ transitions. At the same time, it provides additional confidence for applying the ELA to reactions where the NREFT is no longer applicable, such as in case of charmonium decays into light final states.

The situation is different for the $S P$ transitions. Most of these transitions are expected to be dominated by "short-range" physics, i.e. the tree-level contributions, in the NREFT. From the effective field theory point of view, the tree-level amplitudes serve two important duties: On the one hand, they provide a way to include the short-ranged quark dynamics. On the other hand, they may absorb the ultra-violet behavior of the meson loops and, if necessary, absorb their divergences. In the ELA, part of the short-range physics, which is completely omitted so far in the NREFT, is parameterized by the form factors. Thus, for the $S P$ transitions we expect, and indeed find, that there are quantitative differences between these two approaches. However, it is important to note that the qualitative role of the loops is the same in both treatments.

\section{Summary}

In this paper, the effects of charmed meson loops in the transitions between two charmonia with the emission of one pion or eta have been systematically investigated. The power counting for the loop amplitudes within the framework of a non-relativistic effective field theory is given. The difference among the power counting estimates for the ratios of tree-level and loop contributions for the various transitions considered comes from the quantum number difference of the involved charmonia. It is found that the loops are enhanced in the transitions between two $S$-wave charmonia by a factor of $1 / v$ and in the transitions between two $P$-wave charmonia by a factor of $1 / v^{3}$. As a result, even if the $X(3872)$ is a $c \bar{c}$ charmonium with $J^{P C}=1^{++}$, the dominant contribution to its decays into $\chi_{c J} \pi^{0}$ is given by the intermediate charmed meson loops rather than the tree-level ones. For the transitions between one

$S$-wave and one $P$-wave charmonia, because of the competition between $1 / v^{3}$ and $q^{2} / M_{D}^{2}$ one should analyze case by case. The loops are highly suppressed for the decays $\psi^{\prime} \rightarrow h_{c} \pi^{0}$ and $\eta_{c}^{\prime} \rightarrow \chi_{c 0} \pi^{0}$ which have a small phase space, while the suppression for the decay $\chi_{c 0}^{\prime} \rightarrow \eta_{c}^{\prime} \pi^{0}$ is more moderate, and they are even enhanced in the decay $h_{c}^{\prime} \rightarrow \psi^{\prime} \pi^{0}$.

Among the loop dominated transitions, predictions for the ratios among the decay widths are given. In these ratios, the dependence of the unknown coupling constants is canceled. A detailed calculation in the framework of an effective Lagrangian approach is also given in comparison with the NREFT results. We find that the results from these two methods are qualitatively consistent with each other. Significant deviations appeared only when the results became sensitive to the particular form of the form factors used in the ELA, pointing at possible improvements for this approach, or when the considered transition is dominated by short-range physics. Although the lack of constraints on the structure of the counterterms would lead to uncertainties with the absolute magnitudes of the partial decay widths, the ratios between related channels are less sensitive to such uncertainties, and model-independent 
aspects of the meson loop contributions can be highlighted. Those loop-dominant channels should be testable experimentally in the future, for instance, with Belle, $\bar{P}$ ANDA, BES-III, LHC-b and Super B factory.

\section{Acknowledgments}

We would like to thank Yu Jia, Thomas Mannel and Joan Soto for useful discussions. F.-K.G., C.H. and U.-G.M. would like to thank from the Helmholtz Association through funds provided to the virtual institute "Spin and strong QCD" (VH-VI-231) and by the DFG (SFB/TR 16, "Subnuclear Structure of Matter"), and the European Community-Research Infrastructure Integrating Activity "Study of Strongly Interacting Matter" (acronym HadronPhysics2, Grant Agreement n. 227431) under the Seventh Framework Programme of EU. U.-G.M. also thanks the BMBF for support (grant 06BN9006). Q.Z. and G.L. acknowledge the supports, in part, from the National Natural Science Foundation of China (Grants No. 10675131, 10491306 and 10947007), Chinese Academy of Sciences (KJCX3-SYW-N2), and Ministry of Science and Technology of China (2009CB825200).

\section{A Loop functions in dimensional regularization}

Define the basic three-point scalar loop function in $d$-dimension as

$$
I(q) \equiv i \int \frac{d^{d} l}{(2 \pi)^{d}} \frac{1}{\left(l^{2}-m_{1}^{2}+i \epsilon\right)\left[(P-l)^{2}-m_{2}^{2}+i \epsilon\right]\left[(l-q)^{2}-m_{3}^{2}+i \epsilon\right]} .
$$

Non-relativistically, in the rest frame of the initial particle, i.e. $P^{\mu}=\{M, \overrightarrow{0}\}$, it can be worked out analytically by performing the contour integration over $l^{0}$, and then integrating over the spatial components of the loop momentum using dimensional regularization,

$$
\begin{aligned}
I(q) & =\frac{-i}{8 m_{1} m_{2} m_{3}} \int \frac{d^{d} l}{(2 \pi)^{d}} \frac{1}{\left(l^{0}-\frac{\vec{l}^{2}}{m_{1}}+i \epsilon\right)\left(l^{0}+b_{12}+\frac{\vec{l}^{2}}{m_{2}}-i \epsilon\right)\left[l^{0}+b_{12}-b_{23}-\frac{(\vec{l}-\vec{q})^{2}}{m_{3}}+i \epsilon\right]} \\
& =\frac{\mu_{12} \mu_{23}}{2 m_{1} m_{2} m_{3}} \int \frac{d^{d-1} l}{(2 \pi)^{d-1}} \frac{1}{\left(\vec{l}^{2}+c-i \epsilon\right)\left(\vec{l}^{2}-\frac{2 \mu_{23}}{m_{3}} \vec{l} \cdot \vec{q}+c^{\prime}-i \epsilon\right)} \\
& =\frac{\mu_{12} \mu_{23}}{2 m_{1} m_{2} m_{3}} \int_{0}^{1} d x \int \frac{d^{d-1} l}{(2 \pi)^{d-1}} \frac{1}{\left[\vec{l}^{2}-a x^{2}+\left(c^{\prime}-c\right) x+c-i \epsilon\right]^{2}} \\
& =\frac{\mu_{12} \mu_{23}}{16 \pi m_{1} m_{2} m_{3}} \frac{1}{\sqrt{a}}\left[\tan ^{-1}\left(\frac{c^{\prime}-c}{2 \sqrt{a c}}\right)+\tan ^{-1}\left(\frac{2 a+c-c^{\prime}}{2 \sqrt{a\left(c^{\prime}-a\right)}}\right)\right],
\end{aligned}
$$

where $\mu_{i j}=m_{i} m_{j} /\left(m_{i}+m_{j}\right)$ are the reduced masses, $b_{12}=m_{1}+m_{2}-M, b_{23}=m_{2}+m_{3}+$ $q^{0}-M$, and

$$
a=\left(\frac{\mu_{23}}{m_{3}}\right)^{2} \vec{q}^{2}, \quad c=2 \mu_{12} b_{12}, \quad c^{\prime}=2 \mu_{23} b_{23}+\frac{\mu_{23}}{m_{3}} \vec{q}^{2} .
$$


Since there is no pole for $d=4$, in the last step, we have taken $d=4$.

We also need the vector and tensor loop integrals which are defined as

$$
q^{i} I^{(1)}(q) \equiv i \int \frac{d^{d} l}{(2 \pi)^{d}} \frac{l^{i}}{\left(l^{2}-m_{1}^{2}+i \epsilon\right)\left[(P-l)^{2}-m_{2}^{2}+i \epsilon\right]\left[(l-q)^{2}-m_{3}^{2}+i \epsilon\right]},
$$

and

$q^{i} q^{j} I_{0}^{(2)}(q)+\delta^{i j} \vec{q}^{2} I_{1}^{(2)}(q) \equiv i \int \frac{d^{d} l}{(2 \pi)^{d}} \frac{l^{i} l^{j}}{\left(l^{2}-m_{1}^{2}+i \epsilon\right)\left[(P-l)^{2}-m_{2}^{2}+i \epsilon\right]\left[(l-q)^{2}-m_{3}^{2}+i \epsilon\right]}$.

By using the technique of tensor reduction, we get

$$
\begin{aligned}
I^{(1)}(q) & =\frac{\mu_{23}}{a m_{3}}\left[B\left(c^{\prime}-a\right)-B(c)+\frac{1}{2}\left(c^{\prime}-c\right) I(q)\right], \\
\vec{q}^{2} I_{0}^{(2)}(q) & =\frac{d-3}{d-2} B\left(c^{\prime}-a\right)+\frac{c}{d-2} I(q)+\frac{d-1}{d-2}\left(c^{\prime}-c\right) \frac{m_{3}}{2 \mu_{23}} I^{(1)}(q), \\
\vec{q}^{2} I_{1}^{(2)}(q) & =\frac{1}{d-2} B\left(c^{\prime}-a\right)-\frac{c}{d-2} I(q)-\frac{1}{d-2}\left(c^{\prime}-c\right) \frac{m_{3}}{2 \mu_{23}} I^{(1)}(q),
\end{aligned}
$$

where the function $B(c)$ is defined as

$$
\begin{aligned}
\frac{4 m_{1} m_{2} m_{3}}{\mu_{12} \mu_{23}} B(c) & \equiv \int \frac{d^{d-1} l}{(2 \pi)^{d-1}} \frac{1}{\vec{l}^{2}+c-i \epsilon} \\
& =(4 \pi)^{(1-d) / 2} \Gamma\left(\frac{3-d}{2}\right)(c-i \epsilon)^{(d-3) / 2} \\
& =-\frac{\sqrt{c-i \epsilon}}{4 \pi} .
\end{aligned}
$$

In the last step, the dimension $d$ is taken to be 4 .

\section{B Amplitudes for the charmonium transitions}

Here we give the amplitudes for the transitions between charmonia with the emission of one pion or $\eta$ discussed in the paper. Notice that the expressions for the amplitudes are only given for the charged charmed meson loops. The expressions for the neutral or strange charmed meson loops can be obtained easily by replacing the charged charmed meson masses by the the corresponding neutral or charmed ones. To distinguish loops with different $m_{1}, m_{2}$ and $m_{3}$, we write the loop functions as, for instance, $I^{(1)}(q, M 1, M 2, M 3)$ with $M i$ denoting the meson with the mass $m_{i}$ in the following. 


\section{B.1 Transitions between the $S$-wave charmonia}

- $\psi^{\prime} \rightarrow J / \psi \pi^{0}(\eta)$

$$
\begin{aligned}
& -i \mathcal{M}\left(\psi^{\prime} \rightarrow J / \psi \phi\right)_{ \pm} \\
= & i 2 \frac{g}{F} g_{2} g_{2}^{\prime} \epsilon^{i j k} q^{i} \varepsilon^{j}\left(\psi^{\prime}\right) \varepsilon^{k}(J / \psi) \vec{q}^{2}\left[2 I_{1}^{(2)}\left(q, D^{ \pm}, D^{ \pm}, D^{* \pm}\right)+2 I_{1}^{(2)}\left(q, D^{* \pm}, D^{ \pm}, D^{ \pm}\right)\right. \\
& +4 I_{1}^{(2)}\left(q, D^{ \pm}, D^{* \pm}, D^{* \pm}\right)+2 I_{0}^{(2)}\left(q, D^{ \pm}, D^{* \pm}, D^{* \pm}\right)-I^{(1)}\left(q, D^{ \pm}, D^{* \pm}, D^{* \pm}\right) \\
& +4 I_{1}^{(2)}\left(q, D^{* \pm}, D^{* \pm}, D^{ \pm}\right)+2 I_{0}^{(2)}\left(q, D^{* \pm}, D^{* \pm}, D^{ \pm}\right)-I^{(1)}\left(q, D^{* \pm}, D^{* \pm}, D^{ \pm}\right) \\
& -2 I_{1}^{(2)}\left(q, D^{* \pm}, D^{ \pm}, D^{* \pm}\right)-2 I_{0}^{(2)}\left(q, D^{* \pm}, D^{ \pm}, D^{* \pm}\right)+I^{(1)}\left(q, D^{* \pm}, D^{ \pm}, D^{* \pm}\right) \\
& \left.-10 I_{1}^{(2)}\left(q, D^{* \pm}, D^{* \pm}, D^{* \pm}\right)-2 I_{0}^{(2)}\left(q, D^{* \pm}, D^{* \pm}, D^{* \pm}\right)+I^{(1)}\left(q, D^{* \pm}, D^{* \pm}, D^{* \pm}\right)\right] .
\end{aligned}
$$

\section{B.2 Transitions between the $S$ - and $P$-wave charmonia}

- $\psi^{\prime} \rightarrow h_{c} \pi^{0}$

$$
\begin{aligned}
& -i \mathcal{M}\left(\psi^{\prime} \rightarrow h_{c} \pi^{0}\right)_{ \pm} \\
= & -i 2 \frac{g}{F} g_{1} g_{2}^{\prime}\left\{\vec { q } \cdot \vec { \varepsilon } ( \psi ^ { \prime } ) \vec { q } \cdot \vec { \varepsilon } ( h _ { c } ) \left[I^{(1)}\left(q, D^{ \pm}, D^{ \pm}, D^{* \pm}\right)-I^{(1)}\left(q, D^{* \pm}, D^{ \pm}, D^{* \pm}\right)\right.\right. \\
& \left.+I^{(1)}\left(q, D^{ \pm}, D^{* \pm}, D^{* \pm}\right)-I^{(1)}\left(q, D^{* \pm}, D^{* \pm}, D^{* \pm}\right)\right] \\
& +\vec{q}^{2} \vec{\varepsilon}\left(\psi^{\prime}\right) \cdot \vec{\varepsilon}\left(h_{c}\right)\left[I^{(1)}\left(q, D^{* \pm}, D^{ \pm}, D^{* \pm}\right)+I^{(1)}\left(q, D^{* \pm}, D^{* \pm}, D^{ \pm}\right)\right. \\
& \left.\left.-I^{(1)}\left(q, D^{ \pm}, D^{* \pm}, D^{* \pm}\right)-I^{(1)}\left(q, D^{* \pm}, D^{* \pm}, D^{* \pm}\right)\right]\right\} .
\end{aligned}
$$

- $\eta_{c}^{\prime} \rightarrow \chi_{c 0} \pi^{0}$

$$
\begin{aligned}
-i \mathcal{M}\left(\eta_{c}^{\prime} \rightarrow \chi_{c 0} \pi^{0}\right)_{ \pm}= & -i \frac{2}{\sqrt{3}} \frac{g}{F} g_{1} g_{2}^{\prime} \vec{q}^{2}\left[3 I^{(1)}\left(q, D^{* \pm}, D^{ \pm}, D^{ \pm}\right)\right. \\
& \left.-I^{(1)}\left(q, D^{ \pm}, D^{* \pm}, D^{* \pm}\right)-2 I^{(1)}\left(q, D^{* \pm}, D^{* \pm}, D^{* \pm}\right)\right]
\end{aligned}
$$

- $h_{c} \rightarrow J / \psi \pi^{0}$

$$
\begin{aligned}
& -i \mathcal{M}\left(h_{c} \rightarrow J / \psi \pi^{0}\right)_{ \pm} \\
= & i \frac{g}{F} g_{1} g_{2}\left\{\vec { q } \cdot \vec { \varepsilon } ( h _ { c } ) \vec { q } \cdot \vec { \varepsilon } ( J / \psi ) \left[2 I^{(1)}\left(q, D^{* \pm}, D^{ \pm}, D^{ \pm}\right)-I\left(q, D^{* \pm}, D^{ \pm}, D^{ \pm}\right)\right.\right. \\
& -2 I^{(1)}\left(q, D^{* \pm}, D^{ \pm}, D^{* \pm}\right)+I\left(q, D^{* \pm}, D^{ \pm}, D^{* \pm}\right) \\
& +2 I^{(1)}\left(q, D^{* \pm}, D^{* \pm}, D^{ \pm}\right)-I\left(q, D^{* \pm}, D^{* \pm}, D^{ \pm}\right) \\
& \left.-2 I^{(1)}\left(q, D^{* \pm}, D^{* \pm}, D^{* \pm}\right)+I\left(q, D^{* \pm}, D^{* \pm}, D^{* \pm}\right)\right] \\
& +\vec{q}^{2} \vec{\varepsilon}\left(\psi^{\prime}\right) \cdot \vec{\varepsilon}\left(h_{c}\right)\left[2 I^{(1)}\left(q, D^{* \pm}, D^{ \pm}, D^{* \pm}\right)-I\left(q, D^{* \pm}, D^{ \pm}, D^{* \pm}\right)\right. \\
& +2 I^{(1)}\left(q, D^{ \pm}, D^{* \pm}, D^{* \pm}\right)-I\left(q, D^{ \pm}, D^{* \pm}, D^{* \pm}\right) \\
& -2 I^{(1)}\left(q, D^{* \pm}, D^{* \pm}, D^{ \pm}\right)+I\left(q, D^{* \pm}, D^{* \pm}, D^{ \pm}\right) \\
& \left.\left.-2 I^{(1)}\left(q, D^{* \pm}, D^{* \pm}, D^{* \pm}\right)+I\left(q, D^{* \pm}, D^{* \pm}, D^{* \pm}\right)\right]\right\} .
\end{aligned}
$$


- $\chi_{c 0} \rightarrow \eta_{c} \pi^{0}$

$$
\begin{aligned}
-i \mathcal{M}\left(\chi_{c 0} \rightarrow \eta_{c} \pi^{0}\right)_{ \pm}= & -\frac{i}{\sqrt{3}} \frac{g}{F} g_{1} g_{2} \vec{q}^{2}\left[3 I\left(q, D^{ \pm}, D^{ \pm}, D^{* \pm}\right)-6 I^{(1)}\left(q, D^{ \pm}, D^{ \pm}, D^{* \pm}\right)\right. \\
& -I\left(q, D^{* \pm}, D^{* \pm}, D^{ \pm}\right)+2 I^{(1)} I\left(q, D^{* \pm}, D^{* \pm}, D^{ \pm}\right) \\
& \left.-2 I\left(q, D^{* \pm}, D^{* \pm}, D^{* \pm}\right)+4 I^{(1)}\left(q, D^{* \pm}, D^{* \pm}, D^{* \pm}\right)\right] .
\end{aligned}
$$

\section{B.3 Transitions between the $P$-wave charmonia}

- $\chi_{c 0}^{\prime} \rightarrow \chi_{c 1} \pi^{0}$

$$
\begin{aligned}
-i \mathcal{M}\left(\chi_{c 0}^{\prime} \rightarrow \chi_{c 1} \pi^{0}\right)_{ \pm}= & -i \frac{2}{\sqrt{6}} \frac{g}{F} g_{1} g_{1}^{\prime} \vec{q} \cdot \vec{\varepsilon}\left(\chi_{c 1}\right) \\
& \times\left[3 I\left(q, D^{ \pm}, D^{ \pm}, D^{* \pm}\right)+I\left(q, D^{* \pm}, D^{* \pm}, D^{ \pm}\right)\right] .
\end{aligned}
$$

- $\chi_{c 1}^{\prime} \rightarrow \chi_{c 0} \pi^{0}$

$$
\begin{aligned}
-i \mathcal{M}\left(\chi_{c 1}^{\prime} \rightarrow \chi_{c 0} \pi^{0}\right)_{ \pm}= & i \frac{2}{\sqrt{6}} \frac{g}{F} g_{1} g_{1}^{\prime} \vec{q} \cdot \vec{\varepsilon}\left(\chi_{c 1}^{\prime}\right) \\
& \times\left[3 I\left(q, D^{* \pm}, D^{ \pm}, D^{ \pm}\right)+I\left(q, D^{ \pm}, D^{* \pm}, D^{* \pm}\right)\right] .
\end{aligned}
$$

- $\chi_{c 1}^{\prime} \rightarrow \chi_{c 1} \pi^{0}$

$$
-i \mathcal{M}\left(\chi_{c 1}^{\prime} \rightarrow \chi_{c 1} \pi^{0}\right)_{ \pm}=-i 2 \frac{g}{F} g_{1} g_{1}^{\prime} \epsilon^{i j k} q^{i} \varepsilon^{j}\left(\chi_{c 1}^{\prime}\right) \varepsilon^{k}\left(\chi_{c 1}\right) I\left(q, D^{* \pm}, D^{ \pm}, D^{* \pm}\right) .
$$

- $\chi_{c 1}^{\prime} \rightarrow \chi_{c 2} \pi^{0}$

$$
-i \mathcal{M}\left(\chi_{c 1}^{\prime} \rightarrow \chi_{c 2} \pi^{0}\right)_{ \pm}=i 2 \sqrt{2} \frac{g}{F} g_{1} g_{1}^{\prime} \varepsilon^{i j}\left(\chi_{c 2}\right) q^{i} \varepsilon^{j}\left(\chi_{c 1}^{\prime}\right) I\left(q, D^{ \pm}, D^{* \pm}, D^{* \pm}\right)
$$

where $\varepsilon^{i j}\left(\chi_{c 2}\right)$ is the polarization tensor of the $\chi_{c 2}$, and it is traceless, i.e. $\varepsilon^{i i}\left(\chi_{c 2}\right)=0$, and symmetric.

- $\chi_{c 2}^{\prime} \rightarrow \chi_{c 1} \pi^{0}$

$$
-i \mathcal{M}\left(\chi_{c 2}^{\prime} \rightarrow \chi_{c 1} \pi^{0}\right)_{ \pm}=-i 2 \sqrt{2} \frac{g}{F} g_{1} g_{1}^{\prime} \varepsilon^{i j}\left(\chi_{c 2}\right) q^{i} \varepsilon^{j}\left(\chi_{c 1}^{\prime}\right) I\left(q, D^{* \pm}, D^{* \pm}, D^{ \pm}\right) .
$$

- $\chi_{c 2}^{\prime} \rightarrow \chi_{c 2} \pi^{0}$

$$
-i \mathcal{M}\left(\chi_{c 2}^{\prime} \rightarrow \chi_{c 2} \pi^{0}\right)_{ \pm}=-i 4 \frac{g}{F} g_{1} g_{1}^{\prime} \epsilon^{i j k} q^{i} \varepsilon^{j l}\left(\chi_{c 2}^{\prime}\right) \varepsilon^{k l}\left(\chi_{c 2}\right) I\left(q, D^{* \pm}, D^{* \pm}, D^{* \pm}\right)
$$

- $h_{c}^{\prime} \rightarrow h_{c} \pi^{0}$

$$
\begin{aligned}
-i \mathcal{M}\left(h_{c}^{\prime} \rightarrow h_{c} \pi^{0}\right)_{ \pm}= & -i \frac{g}{F} g_{1} g_{1}^{\prime} \epsilon^{i j k} q^{i} \varepsilon^{j}\left(h_{c}^{\prime}\right) \varepsilon^{k}\left(h_{c}\right) \\
& \times\left[I\left(q, D^{* \pm}, D^{ \pm}, D^{* \pm}\right)+I\left(q, D^{ \pm}, D^{* \pm}, D^{* \pm}\right)\right. \\
& \left.-I\left(q, D^{* \pm}, D^{* \pm}, D^{ \pm}\right)+I\left(q, D^{* \pm}, D^{* \pm}, D^{* \pm}\right)\right] .
\end{aligned}
$$




\section{The Effective Lagrangian Approach (ELA)}

\section{C.1 Effective Lagrangians}

Based on the heavy quark symmetry [69, 70], the relevant effective Lagrangians used here are:

$$
\begin{aligned}
& \mathcal{L}_{1}=i \frac{g_{1}}{2} \operatorname{Tr}\left[P_{c \bar{c}}^{\mu} \bar{H}_{2 i} \gamma_{\mu} \bar{H}_{1 i}\right]+\text { H.c., } \\
& \mathcal{L}_{2}=i \frac{g_{2}}{2} \operatorname{Tr}\left[R_{c \bar{c}} \bar{H}_{2 i} \gamma^{\mu} \stackrel{\leftrightarrow}{\partial}_{\mu} \bar{H}_{1 i}\right]+\text { H.c. },
\end{aligned}
$$

where the spin multiplets for these four $P$-wave and two $S$-wave charmonium states are expressed as

$$
\begin{aligned}
P_{c \bar{c}}^{\mu} & =\left(\frac{1+\psi}{2}\right)\left(\chi_{c 2}^{\mu \alpha} \gamma_{\alpha}+\frac{1}{\sqrt{2}} \epsilon^{\mu \nu \alpha \beta} v_{\alpha} \gamma_{\beta} \chi_{c 1 \nu}+\frac{1}{\sqrt{3}}\left(\gamma^{\mu}-v^{\mu}\right) \chi_{c 0}+h_{c}^{\mu} \gamma_{5}\right)\left(\frac{1-\psi}{2}\right), \\
R_{c \bar{c}} & =\left(\frac{1+\psi}{2}\right)\left(\psi^{\mu} \gamma_{\mu}-\eta_{c} \gamma_{5}\right)\left(\frac{1-\psi}{2}\right) .
\end{aligned}
$$

The charmed and anti-charmed meson triplets read

$$
\begin{aligned}
& H_{1 i}=\left(\frac{1+\psi}{2}\right)\left[\mathcal{D}_{i}^{* \mu} \gamma_{\mu}-\mathcal{D}_{i} \gamma_{5}\right], \\
& H_{2 i}=\left[\overline{\mathcal{D}}_{i}^{* \mu} \gamma_{\mu}-\overline{\mathcal{D}}_{i} \gamma_{5}\right]\left(\frac{1-\not}{2}\right),
\end{aligned}
$$

where $\mathcal{D}$ and $\mathcal{D}^{*}$ denote the pseudoscalar and vector charmed meson fields, respectively, i.e. $\mathcal{D}^{(*)}=\left(D^{0(*)}, D^{+(*)}, D_{s}^{+(*)}\right)$. These Lagrangians can be reduced to the two-component ones used in the NREFT in Section 3 ,

Consequently, the Lagrangian for $S$-wave $J / \psi$ and $\eta_{c}$ is

$$
\begin{aligned}
& \mathcal{L}_{S}=i g_{\psi D^{*} D^{*}}\left(-\psi^{\mu} D^{* \nu} \overleftrightarrow{\partial}_{\mu} D_{\nu}^{* \dagger}+\psi^{\mu} D^{* \nu} \partial_{\nu} D_{\mu}^{* \dagger}-\psi_{\mu} \partial_{\nu} D^{* \mu} D^{* \nu \dagger}\right) \\
& +i g_{\psi D D} \psi_{\mu}\left(\partial^{\mu} D D^{\dagger}-D \partial^{\mu} D^{\dagger}\right)+g_{\psi D D} \varepsilon^{\mu \nu \alpha \beta} \partial_{\mu} \psi_{\nu}\left(D_{\alpha}^{*} \overleftrightarrow{\partial}_{\beta} D^{\dagger}-D \overleftrightarrow{\partial}_{\beta} D_{\alpha}^{* \dagger}\right)
\end{aligned}
$$

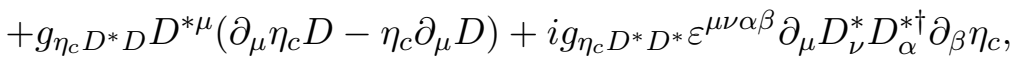

and the Lagrangian for $P$-wave $h_{c}$ and $\chi_{c J}$ is

$$
\begin{aligned}
\mathcal{L}_{P}= & i g_{\chi_{c 2} D^{*} D^{*}} \chi_{c 2}^{\alpha \beta} D_{\alpha}^{*} D_{\beta}^{* \dagger}+g_{\chi_{c 1} D^{*} D} \chi_{c 1 \mu} D^{* \mu} D+i g_{\chi_{c 0} D^{*} D^{*}} D^{* \mu} D_{\mu}^{* \dagger}+i g_{\chi_{c 0} D D} D D \\
& +g_{h_{c} D^{*} D} D^{* \mu} h_{c \mu}+i g_{h_{c} D^{*} D^{*} \varepsilon^{\mu \nu \alpha \beta} D_{\mu}^{*} \partial_{\alpha} h_{c \nu} D_{\beta}^{*}+\text { H.c. }}
\end{aligned}
$$

where the couplings are

$$
\begin{aligned}
g_{\psi D D} & =g_{2} \sqrt{m_{\psi}} m_{D}, \quad g_{\psi D^{*} D^{*}}=-g_{2} \sqrt{m_{\psi}} m_{D^{*}}, \quad g_{\psi D^{*} D}=g_{2} \sqrt{\frac{m_{\psi} m_{D}}{m_{D^{*}}}} \\
g_{\eta_{c} D^{*} D} & =g_{2} \sqrt{m_{\eta_{c}}} m_{D}, \quad g_{\eta_{c} D^{*} D^{*}}=g_{2} \sqrt{\frac{m_{\eta_{c}} m_{D^{*}}}{m_{D^{*}}}} \\
g_{h_{c} D^{*} D} & =-g_{1} \sqrt{m_{h_{c}} m_{D m_{D^{*}}}}, \quad g_{h_{c} D^{*} D^{*}}=g_{1} \sqrt{\frac{m_{D^{*}}^{2}}{m_{h_{c}}}}
\end{aligned}
$$




$$
\begin{aligned}
g_{\chi_{c 0} D D} & =-\sqrt{3} g_{1} \sqrt{m_{\chi_{c 0}}} m_{D}, \quad g_{\chi_{c 0} D^{*} D^{*}}=-\frac{1}{\sqrt{3}} g_{1} \sqrt{m_{\chi_{c 0}}} m_{D}^{*}, \\
g_{\chi_{c 1} D^{*} D} & =\sqrt{2} g_{1} \sqrt{m_{D} m_{D}^{*} m_{\chi_{c 0}}}, \quad g_{\chi_{c 2} D^{*} D^{*}}=g_{1} m_{D^{*}} \sqrt{m_{\chi_{c 2}}} \\
g_{D^{*} D^{*} \pi} & =\frac{g_{D^{*} D \pi}}{\sqrt{m_{D^{m} m_{D^{*}}}}}=\frac{\sqrt{2}}{F} g .
\end{aligned}
$$

In the following, we present the transition amplitudes for the intermediate meson loops listed in Table 3 in the framework of the ELA. Some conventions should be clarified in advance. Notice that the expressions are similar for the charged, neutral and charmed strange mesons except that different charmed meson masses are applied. We thus only present the amplitudes for those charged charmed meson loops. $G_{1} G_{2} G_{3}$ is the product of vertex couplings for each loop, and the explicit expression can be found in Eq. (C.21). $\mathcal{F}=\prod_{i} \mathcal{F}_{i}\left(m_{i}, q_{i}^{2}\right)$ is the form factor. $\left[q_{1}, q_{3}, q_{2}\right]$ are the four-vector momenta for the intermediate mesons [M1, M3, M2], respectively. $p_{i}\left(p_{f}\right), \varepsilon_{i}\left(\varepsilon_{f}\right)$, and $\phi_{i}\left(\phi_{f}\right)$ are the initial (final) charmonium four-vector momentum, polarization vector, and polarization tensor, respectively. $p_{2}$ is the four-vector momentum of $\pi^{0}$ or $\eta$.

\section{C.2 Transitions between the $S$-wave charmonia}

- $\psi^{\prime} \rightarrow J / \psi \pi^{0}(\eta)$

$$
\begin{aligned}
\mathcal{M}_{\left[D D D^{*}\right]}= & -2 G_{1} G_{2} G_{3} \varepsilon_{\alpha \beta \mu \nu} \varepsilon_{i \rho} p_{f}^{\alpha} \varepsilon_{f}^{* \beta} p_{2}^{\nu} \int \frac{d^{4} q_{2}}{(2 \pi)^{4}} \frac{\mathcal{F}}{a_{1} a_{2} a_{3}} q_{1}^{\rho} q_{2}^{\mu}, \\
\mathcal{M}_{\left[D D^{*} D\right]}= & -2 G_{1} G_{2} G_{3} \varepsilon_{\alpha \beta \mu \nu} p_{i}^{\alpha} \varepsilon_{i}^{\beta} \varepsilon_{f \rho}^{*} p_{2}^{\nu} \int \frac{d^{4} q_{2}}{(2 \pi)^{4}} \frac{\mathcal{F}}{a_{1} a_{2} a_{3}} q_{3}^{\mu} q_{2}^{\rho}, \\
\mathcal{M}_{\left[D D^{*} D^{*}\right]}= & G_{1} G_{2} G_{3} \varepsilon_{\alpha \beta \mu \nu} \varepsilon_{\rho \sigma \tau \lambda} \varepsilon_{\alpha^{\prime} \beta^{\prime} \mu^{\prime} \nu^{\prime}} p_{i}^{\alpha} \varepsilon_{i}^{\beta} p_{f}^{\rho} \varepsilon_{f}^{* \sigma} p_{2}^{\mu^{\prime}} g^{\lambda \beta^{\prime}} g^{\nu \nu^{\prime}} \int \frac{d^{4} q_{2}}{(2 \pi)^{4}} \frac{\mathcal{F}}{a_{1} a_{2} a_{3}} q_{3}^{\mu} q_{2}^{\tau} q_{2}^{\alpha^{\prime}}, \\
\mathcal{M}_{\left[D^{*} D D^{*}\right]}= & G_{1} G_{2} G_{3} \varepsilon_{\alpha \beta \mu \nu} p_{i}^{\alpha} \varepsilon_{i}^{\beta} p_{2 \rho} \int \frac{d^{4} q_{2}}{(2 \pi)^{4}} \frac{\mathcal{F}}{a_{1} a_{2} a_{3}} q_{1}^{\mu}\left[-\varepsilon_{f}^{* \nu} q_{1 \sigma}\left(-g^{\rho \sigma}+\frac{q_{2}^{\rho} q_{2}^{\sigma}}{m_{D^{*}}^{2}}\right)\right. \\
& \left.+2 \varepsilon_{f \sigma}^{*} q_{1}^{\sigma}\left(-g^{\nu \rho}+\frac{q_{2}^{\nu} q_{2}^{\rho}}{m_{D^{*}}^{2}}\right)+\varepsilon_{f \sigma}^{*} q_{2}^{\nu}\left(-g^{\rho \sigma}+\frac{q_{2}^{\rho} q_{2}^{\sigma}}{m_{D^{*}}^{2}}\right)\right], \\
\mathcal{M}_{\left[D^{*} D^{*} D\right]=} & G_{1} G_{2} G_{3} \varepsilon_{\alpha \beta \mu \nu} p_{f}^{\alpha} \varepsilon_{f}^{* \beta} p_{2 \rho} \int \frac{d^{4} q_{2}}{(2 \pi)^{4}} \frac{\mathcal{F}}{a_{1} a_{2} a_{3}} q_{1}^{\mu}\left[-\varepsilon_{i}^{\nu} q_{1 \sigma}\left(-g^{\rho \sigma}+\frac{q_{3}^{\sigma} q_{3}^{\rho}}{m_{D^{*}}^{2}}\right)\right. \\
& \left.+2 \varepsilon_{i \sigma} q_{1}^{\sigma}\left(-g^{\nu \rho}+\frac{q_{3}^{\nu} q_{3}^{\rho}}{m_{D^{*}}^{2}}\right)+\varepsilon_{i \sigma} q_{3}^{\nu}\left(-g^{\rho \sigma}+\frac{q_{3}^{\rho} q_{3}^{\sigma}}{m_{D^{*}}^{2}}\right)\right], \\
\mathcal{M}_{\left[D^{*} D^{*} D^{*}\right]=} & G_{1} G_{2} G_{3} \varepsilon_{\alpha \beta \mu \nu} \int \frac{d^{4} q_{2}}{(2 \pi)^{4}} \frac{\mathcal{F}}{a_{1} a_{2} a_{3}} q_{3}^{\alpha} q_{2}^{\mu}\left[2 \varepsilon_{i}^{\nu} p_{i}^{\beta} \varepsilon_{f}^{*} \cdot q_{1}-2 \varepsilon_{i} \cdot q_{1} \varepsilon_{f}^{\beta} p_{i}^{\nu}\right. \\
- & p_{i}^{\beta} \varepsilon_{f}^{\nu}\left(-\varepsilon_{i} \cdot q_{2}+\frac{\varepsilon_{i} \cdot q_{2} q_{2}^{2}}{m_{D^{*}}^{2}}\right)-4 \varepsilon_{i} \cdot q_{1} \varepsilon_{f} \cdot q_{1} g^{\beta \nu}-2 \varepsilon_{i} \cdot q_{1} \varepsilon_{f}^{\nu} q_{2}^{\beta} \\
+ & \left.\varepsilon_{i}^{\beta} p_{i}^{\nu}\left(-\varepsilon_{f} \cdot q_{3}+\frac{q_{1} \cdot q_{3} \varepsilon_{f} \cdot q_{1}}{m_{D^{*}}^{2}}\right)+2 \varepsilon_{i}^{\beta} \varepsilon_{f}^{\nu}\left(-q_{2} \cdot q_{3}+\frac{q_{2}^{2} q_{2} \cdot q_{3}}{m_{D^{*}}^{2}}\right)\right] .
\end{aligned}
$$




\section{C.3 Transitions between the $S$ - and $P$-wave charmonia}

- $\psi^{\prime} \rightarrow h_{c} \pi^{0}$

$$
\begin{aligned}
\mathcal{M}_{\left[D D^{*} D\right]} & =2 G_{1} G_{2} G_{3} \varepsilon_{i \alpha} \varepsilon_{f \beta}^{*} p_{2 \mu} \int \frac{d^{4} q_{2}}{(2 \pi)^{4}} \frac{\mathcal{F}}{a_{1} a_{2} a_{3}} q_{1}^{\alpha}\left(-g^{\beta \mu}+\frac{q_{3}^{\beta} q_{3}^{\mu}}{m_{D^{*}}^{2}}\right), \\
\mathcal{M}_{\left[D D^{*} D^{*}\right]} & =2 G_{1} G_{2} G_{3} \varepsilon_{\alpha \beta \mu \nu} \varepsilon_{\rho \sigma \tau} \lambda p_{i}^{\alpha} \varepsilon_{i}^{\beta} \varepsilon_{f}^{* \sigma} g^{\nu \lambda} p_{2}^{\tau} \int \frac{d^{4} q_{2}}{(2 \pi)^{4}} \frac{\mathcal{F}}{a_{1} a_{2} a_{3}} q_{3}^{\mu} q_{2}^{\rho}, \\
\mathcal{M}_{\left[D^{*} D D^{*}\right]} & =G_{1} G_{2} G_{3} \varepsilon_{\alpha \beta \mu \nu} \varepsilon_{\rho \sigma \tau \lambda} p_{i}^{\alpha} \varepsilon_{i}^{\beta} p_{f}^{\rho} \varepsilon_{f}^{* \sigma} p_{2 \xi}\left(-g^{\mu \tau}\right) \int \frac{d^{4} q_{2}}{(2 \pi)^{4}} \frac{\mathcal{F}}{a_{1} a_{2} a_{3}} q_{1}^{\mu}\left(-g^{\nu \xi}+\frac{q_{2}^{\nu} q_{2}^{\xi}}{m_{D^{*}}^{2}}\right), \\
\mathcal{M}_{\left[D^{*} D^{*} D\right]}= & G_{1} G_{2} G_{3} \varepsilon_{f \mu}^{*} p_{2 \nu} \varepsilon_{i \alpha} \int \frac{d^{4} q_{2}}{(2 \pi)^{4}} \frac{\mathcal{F}}{a_{1} a_{2} a_{3}}\left[q_{1 \beta}\left(-g^{\alpha \mu}+\frac{q_{1}^{\alpha} q_{1}^{\mu}}{m_{D^{*}}^{2}}\right)\left(-g^{\beta \nu}+\frac{q_{3}^{\beta} q_{3}^{\nu}}{m_{D^{*}}^{2}}\right)\right. \\
& -2 q_{1}^{\alpha}\left(-g^{\beta \mu}+\frac{q_{1}^{\beta} q_{1}^{\mu}}{m_{D^{*}}^{2}}\right)\left(-g_{\beta}^{\nu}+\frac{q_{3 \beta} q_{3}^{\nu}}{m_{D^{*}}^{2}}\right) \\
& \left.-q_{3 \beta}\left(-g^{\alpha \mu}+\frac{q_{3}^{\alpha} q_{3}^{\mu}}{m_{D^{*}}^{2}}\right)\left(-g^{\beta \nu}+\frac{q_{1}^{\beta} q_{1}^{\nu}}{m_{D^{*}}^{2}}\right)\right] \\
\mathcal{M}_{\left[D^{*} D^{*} D^{*}\right]} & =-G_{1} G_{2} G_{3} \varepsilon_{\mu \nu \rho \sigma} \varepsilon_{\tau \lambda \xi \eta} p_{f}^{\mu} p_{2}^{\xi} \varepsilon_{f}^{* \nu} \varepsilon_{i \alpha} g^{\sigma \lambda} \int \frac{d^{4} q_{2}}{(2 \pi)^{4}} \frac{\mathcal{F}}{a_{1} a_{2} a_{3}} q_{2}^{\tau}\left[q_{1} \eta\left(-g^{\alpha \rho}+\frac{q_{1}^{\alpha} q_{1}^{\rho}}{m_{D^{*}}^{2}}\right)\right. \\
& \left.-2 q_{1}^{\alpha}\left(-g^{\eta \rho}+\frac{q_{1}^{\eta} q_{1}^{\rho}}{m_{D^{*}}^{2}}\right)-\left(-q_{3}^{\rho}+\frac{q_{1} \cdot q_{3} q_{1}^{\rho}}{m_{D^{*}}^{2}}\right) g^{\alpha \eta}\right] .
\end{aligned}
$$

- $\eta_{c}^{\prime} \rightarrow \chi_{c 0} \pi^{0}$

$$
\begin{aligned}
\mathcal{M}_{\left[D D D^{*}\right]} & =2 G_{1} G_{2} G_{3} p_{i}^{\alpha} p_{2}^{\beta} \int \frac{d^{4} q_{2}}{(2 \pi)^{4}} \frac{\mathcal{F}}{a_{1} a_{2} a_{3}}\left(-g_{\alpha \beta}+\frac{q_{3 \alpha} q_{3 \beta}}{m_{D^{*}}^{2}}\right), \\
\mathcal{M}_{\left[D^{*} D D^{*}\right]} & =2 G_{1} G_{2} G_{3} p_{i}^{\alpha} p_{2}^{\mu} \int \frac{d^{4} q_{2}}{(2 \pi)^{4}} \frac{\mathcal{F}}{a_{1} a_{2} a_{3}}\left(-g_{\alpha}^{\beta}+\frac{q_{1 \alpha} q_{1}^{\beta}}{m_{D^{*}}^{2}}\right)\left(-g_{\beta \mu}+\frac{q_{2 \beta} q_{2 \mu}}{m_{D^{*}}^{2}}\right), \\
\mathcal{M}_{\left[D^{*} D^{*} D^{*}\right]} & =2 G_{1} G_{2} G_{3} \varepsilon_{\alpha \beta \mu \nu} \varepsilon_{\rho \sigma \tau \lambda} p_{i}^{\mu} p_{2}^{\tau} g^{\beta \sigma} g^{\nu \lambda} \int \frac{d^{4} q_{2}}{(2 \pi)^{4}} \frac{\mathcal{F}}{a_{1} a_{2} a_{3}} q_{1}^{\alpha} q_{2}^{\rho} .
\end{aligned}
$$

- $h_{c} \rightarrow J / \psi \pi^{0}$

$$
\begin{aligned}
\mathcal{M}_{\left[D D^{*} D\right]}= & 2 G_{1} G_{2} G_{3} \varepsilon_{i \alpha} \varepsilon_{f \beta}^{*} p_{2 \mu} \int \frac{d^{4} q_{2}}{(2 \pi)^{4}} \frac{\mathcal{F}}{a_{1} a_{2} a_{3}} q_{1}^{\beta}\left(-g^{\alpha \mu}+\frac{q_{3}^{\alpha} q_{3}^{\mu}}{m_{D^{*}}^{2}}\right), \\
\mathcal{M}_{\left[D^{*} D D^{*}\right]}= & G_{1} G_{2} G_{3} \varepsilon_{i \alpha} p_{2 \beta} \int \frac{d^{4} q_{2}}{(2 \pi)^{4}} \frac{\mathcal{F}}{a_{1} a_{2} a_{3}}\left[\varepsilon_{f \mu}^{*} q_{1 \nu}\left(-g^{\alpha \mu}+\frac{q_{1}^{\alpha} q_{1}^{\mu}}{m_{D^{*}}^{2}}\right)\left(-g^{\beta \nu}+\frac{q_{2}^{\beta} q_{2}^{\nu}}{m_{D^{*}}^{2}}\right)\right. \\
& -2 \varepsilon_{f \mu}^{*} q_{1}^{\mu}\left(-g^{\alpha \nu}+\frac{q_{1}^{\alpha} q_{1}^{\nu}}{m_{D^{*}}^{2}}\right)\left(-g_{\nu}^{\beta}+\frac{q_{2}^{\beta} q_{2 \nu}}{m_{D^{*}}^{2}}\right) \\
& \left.-\varepsilon_{f \mu}^{*} q_{2 \nu}\left(-g^{\alpha \nu}+\frac{q_{1}^{\alpha} q_{1}^{\nu}}{m_{D^{*}}^{2}}\right)\left(-g^{\beta \mu}+\frac{q_{2}^{\beta} q_{2}^{\mu}}{m_{D^{*}}^{2}}\right)\right],
\end{aligned}
$$




$$
\begin{aligned}
\mathcal{M}_{\left[D D^{*} D^{*}\right]}= & 2 G_{1} G_{2} G_{3} \varepsilon_{\alpha \beta \mu \nu} \varepsilon_{\rho \sigma \tau \lambda} p_{f}^{\alpha} \varepsilon_{i}^{\lambda} \varepsilon_{f}^{* \beta} g^{\nu \sigma} p_{2}^{\tau} \int \frac{d^{4} q_{2}}{(2 \pi)^{4}} \frac{\mathcal{F}}{a_{1} a_{2} a_{3}} q_{2}^{\mu} q_{2}^{\rho}, \\
\mathcal{M}_{\left[D^{*} D^{*} D\right]}= & -G_{1} G_{2} G_{3} \varepsilon_{\alpha \beta \mu \nu} \varepsilon_{\rho \sigma \tau \lambda} p_{i}^{\alpha} \varepsilon_{i}^{\beta} p_{f}^{\rho} \varepsilon_{f}^{* \sigma} p_{2 \xi} g^{\mu \lambda} \int \frac{d^{4} q_{2}}{(2 \pi)^{4}} \frac{\mathcal{F}}{a_{1} a_{2} a_{3}} q_{1}^{\tau}\left(-g^{\nu \xi}+\frac{q_{3}^{\nu} q_{3}^{\xi}}{m_{D^{*}}^{2}}\right), \\
\mathcal{M}_{\left[D^{*} D^{*} D\right]=} & -G_{1} G_{2} G_{3} \varepsilon_{\alpha \beta \mu \nu} \varepsilon_{\rho \sigma \tau \lambda} p_{i}^{\alpha} \varepsilon_{i}^{\beta} q_{2}^{\rho} p_{2}^{\tau} g^{\nu \lambda} \\
& \times \int \frac{d^{4} q_{2}}{(2 \pi)^{4}} \frac{\mathcal{F}}{a_{1} a_{2} a_{3}}\left[-q_{1}^{\sigma}\left(-\varepsilon_{f}^{\mu}+\frac{q_{1}^{\mu} q_{1} \cdot \varepsilon_{f}^{*}}{m_{D^{*}}^{2}}\right)\right. \\
& \left.+2 \varepsilon_{f}^{*} \cdot q_{1}\left(-g^{\mu \sigma}+\frac{q_{1}^{\mu} q_{1}^{\sigma}}{m_{D^{*}}^{2}}\right)+\varepsilon_{f}^{* \sigma}\left(-q_{2}^{\mu}+\frac{q_{1}^{\mu} q_{1} \cdot q_{2}}{m_{D^{*}}^{2}}\right)\right] .
\end{aligned}
$$

- $\chi_{c 0} \rightarrow \eta_{c} \pi^{0}$

$$
\begin{aligned}
\mathcal{M}_{\left[D D D^{*}\right]} & =G_{1} G_{2} G_{3} p_{f}^{\alpha} p_{2}^{\beta} \int \frac{d^{4} q_{2}}{(2 \pi)^{4}} \frac{\mathcal{F}}{a_{1} a_{2} a_{3}}\left(-g_{\alpha \beta}+\frac{q_{2 \alpha} q_{2 \beta}}{m_{D^{*}}^{2}}\right), \\
\mathcal{M}_{\left[D^{*} D^{*} D\right]} & =G_{1} G_{2} G_{3} p_{f}^{\beta} p_{2}^{\mu} \int \frac{d^{4} q_{2}}{(2 \pi)^{4}} \frac{\mathcal{F}}{a_{1} a_{2} a_{3}},\left(-g_{\beta}^{\alpha}+\frac{q_{1}^{\alpha} q_{1 \beta}}{m_{D^{*}}^{2}}\right)\left(-g_{\alpha \mu}+\frac{q_{3 \alpha} q_{3 \mu}}{m_{D^{*}}^{2}}\right) \\
\mathcal{M}_{\left[D^{*} D^{*} D^{*}\right]} & =-G_{1} G_{2} G_{3} \varepsilon_{\alpha \beta \mu \nu} \varepsilon_{\rho \sigma \tau \lambda} p_{f}^{\alpha} p_{2}^{\tau} g^{\beta \lambda} g^{\nu \sigma} \int \frac{d^{4} q_{2}}{(2 \pi)^{4}} \frac{\mathcal{F}}{a_{1} a_{2} a_{3}} q_{2}^{\mu} q_{2}^{\rho} .
\end{aligned}
$$

\section{C.4 Transitions between the $P$-wave charmonia}

- $\chi_{c 0}^{\prime} \rightarrow \chi_{c 1} \pi^{0}$

$$
\begin{aligned}
\mathcal{M}_{\left[D^{*} D^{*} D\right]} & =G_{1} G_{2} G_{3} \varepsilon_{f \beta}^{*} p_{2 \mu} \int \frac{d^{4} q_{2}}{(2 \pi)^{4}} \frac{\mathcal{F}}{a_{1} a_{2} a_{3}}\left(-g_{\alpha}^{\beta}+\frac{q_{1 \alpha} q_{1}^{\beta}}{m_{D^{*}}^{2}}\right)\left(-g^{\alpha \mu}+\frac{q_{3}^{\alpha} q_{3}^{\mu}}{m_{D^{*}}^{2}}\right), \\
\mathcal{M}_{\left[D D D^{*}\right]} & =G_{1} G_{2} G_{3} \varepsilon_{f \alpha}^{*} p_{2 \beta} \int \frac{d^{4} q_{2}}{(2 \pi)^{4}} \frac{\mathcal{F}}{a_{1} a_{2} a_{3}}\left(-g^{\alpha \beta}+\frac{q_{2}^{\alpha} q_{2}^{\beta}}{m_{D^{*}}^{2}}\right) .
\end{aligned}
$$

- $\chi_{c 1}^{\prime} \rightarrow \chi_{c 0} \pi^{0}$

$$
\begin{aligned}
\mathcal{M}_{\left[D D^{*} D\right]} & =G_{1} G_{2} G_{3} \varepsilon_{i \alpha} p_{2 \beta} \int \frac{d^{4} q_{2}}{(2 \pi)^{4}} \frac{\mathcal{F}}{a_{1} a_{2} a_{3}}\left(-g^{\alpha \beta}+\frac{q_{3}^{\alpha} q_{3}^{\beta}}{m_{D^{*}}^{2}}\right) \\
\mathcal{M}_{\left[D^{*} D D^{*}\right]} & =G_{1} G_{2} G_{3} \varepsilon_{i \alpha} p_{2 \mu} \int \frac{d^{4} q_{2}}{(2 \pi)^{4}} \frac{\mathcal{F}}{a_{1} a_{2} a_{3}}\left(-g_{\beta}^{\alpha}+\frac{q_{1}^{\alpha} q_{1 \beta}}{m_{D^{*}}^{2}}\right)\left(-g^{\beta \mu}+\frac{q_{2}^{\beta} q_{2}^{\mu}}{m_{D^{*}}^{2}}\right) .
\end{aligned}
$$

- $\chi_{c 1}^{\prime} \rightarrow \chi_{c 1} \pi^{0}$

$$
\mathcal{M}_{\left[D D^{*} D^{*}\right]}=G_{1} G_{2} G_{3} \varepsilon_{\alpha \beta \mu \nu} \varepsilon_{i}^{\beta} \varepsilon_{f}^{* \mu} p_{2}^{\nu} \int \frac{d^{4} q_{2}}{(2 \pi)^{4}} \frac{\mathcal{F}}{a_{1} a_{2} a_{3}} q_{3}^{\alpha} .
$$


- $\chi_{c 1}^{\prime} \rightarrow \chi_{c 2} \pi^{0}$

$$
\begin{aligned}
\mathcal{M}_{\left[D^{*} D D^{*}\right]}= & G_{1} G_{2} G_{3} \phi_{f \alpha \beta}^{*} \varepsilon_{i \mu} p_{2 \nu} \int \frac{d^{4} q_{2}}{(2 \pi)^{4}} \frac{\mathcal{F}}{a_{1} a_{2} a_{3}}\left[\left(-g^{\alpha \mu}+\frac{q_{1}^{\alpha} q_{1}^{\mu}}{m_{D^{*}}^{2}}\right)\left(-g^{\beta \nu}+\frac{q_{2}^{\beta} q_{2}^{\nu}}{m_{D^{*}}^{2}}\right)\right. \\
& \left.+\left(-g^{\beta \mu}+\frac{q_{1}^{\beta} q_{1}^{\mu}}{m_{D^{*}}^{2}}\right)\left(-g^{\alpha \nu}+\frac{q_{2}^{\alpha} q_{2}^{\nu}}{m_{D^{*}}^{2}}\right)\right] .
\end{aligned}
$$

- $\chi_{c 2}^{\prime} \rightarrow \chi_{c 1} \pi^{0}$

$$
\begin{aligned}
\mathcal{M}_{\left[D^{*} D^{*} D\right]}= & G_{1} G_{2} G_{3} \phi_{i \alpha \beta} \varepsilon_{f \mu}^{*} p_{2 \nu} \int \frac{d^{4} q_{2}}{(2 \pi)^{4}} \frac{\mathcal{F}}{a_{1} a_{2} a_{3}}\left[\left(-g^{\alpha \mu}+\frac{q_{1}^{\alpha} q_{1}^{\mu}}{m_{D^{*}}^{2}}\right)\left(-g^{\beta \nu}+\frac{q_{3}^{\beta} q_{3}^{\nu}}{m_{D^{*}}^{2}}\right)\right. \\
& \left.+\left(-g^{\beta \mu}+\frac{q_{1}^{\beta} q_{1}^{\mu}}{m_{D^{*}}^{2}}\right)\left(-g^{\alpha \nu}+\frac{q_{3}^{\alpha} q_{3}^{\nu}}{m_{D^{*}}^{2}}\right)\right] .
\end{aligned}
$$

- $\chi_{c 2}^{\prime} \rightarrow \chi_{c 2} \pi^{0}$

$$
\begin{aligned}
\mathcal{M}_{\left[D^{*} D^{*} D^{*}\right]}= & G_{1} G_{2} G_{3} \phi_{i \alpha \beta} \phi_{f \mu \nu}^{*} \varepsilon_{\rho \sigma \tau \lambda} p_{2}^{\tau} \int \frac{d^{4} q_{2}}{(2 \pi)^{4}} \frac{\mathcal{F}}{a_{1} a_{2} a_{3}} q_{2}^{\rho}\left[g^{\beta \lambda} g^{\nu \sigma}\left(-g^{\alpha \mu}+\frac{q_{1}^{\alpha} q_{1}^{\mu}}{m_{D^{*}}^{2}}\right)\right. \\
& +g^{\beta \lambda} g^{\mu \sigma}\left(-g^{\alpha \nu}+\frac{q_{1}^{\alpha} q_{1}^{\nu}}{m_{D^{*}}^{2}}\right)+g^{\alpha \lambda} g^{\nu \sigma}\left(-g^{\beta \mu}+\frac{q_{1}^{\beta} q_{1}^{\mu}}{m_{D^{*}}^{2}}\right) \\
& \left.+g^{\alpha \lambda} g^{\mu \sigma}\left(-g^{\beta \nu}+\frac{q_{1}^{\beta} q_{1}^{\nu}}{m_{D^{*}}^{2}}\right)\right]
\end{aligned}
$$

- $h_{c}^{\prime} \rightarrow h_{c} \pi^{0}$

$$
\begin{aligned}
\mathcal{M}_{\left[D D^{*} D^{*}\right]}= & G_{1} G_{2} G_{3} \varepsilon_{\alpha \beta \mu \nu} p_{2}^{\mu} \varepsilon_{i}^{\nu} \varepsilon_{f}^{* \beta} \int \frac{d^{4} q_{2}}{(2 \pi)^{4}} \frac{\mathcal{F}}{a_{1} a_{2} a_{3}} q_{2}^{\alpha}, \\
\mathcal{M}_{\left[D^{*} D D^{*}\right]}= & G_{1} G_{2} G_{3} \varepsilon_{\alpha \beta \mu \nu} p_{f}^{\alpha} \varepsilon_{i \rho} \varepsilon_{f}^{* \beta} p_{2 \sigma} \\
& \times \int \frac{d^{4} q_{2}}{(2 \pi)^{4}} \frac{\mathcal{F}}{a_{1} a_{2} a_{3}}\left(-g^{\mu \rho}+\frac{q_{1}^{\mu} q_{1}^{\rho}}{m_{D^{*}}^{2}}\right)\left(-g^{\nu \sigma}+\frac{q_{2}^{\nu} q_{2}^{\sigma}}{m_{D^{*}}^{2}}\right), \\
\mathcal{M}_{\left[D^{*} D^{*} D^{*}\right]}= & G_{1} G_{2} G_{3} \varepsilon_{\alpha \beta \mu \nu} \varepsilon_{\rho \sigma \tau \lambda} \varepsilon_{\alpha^{\prime} \beta^{\prime} \mu^{\prime} \nu^{\prime}} p_{i}^{\alpha} \varepsilon_{i}^{\beta} p_{f}^{\rho} \varepsilon_{f}^{* \sigma} p_{2}^{\mu^{\prime}} g^{\lambda \beta^{\prime}} g^{\nu \nu^{\prime}} \\
& \times \int \frac{d^{4} q_{2}}{(2 \pi)^{4}} \frac{\mathcal{F}}{a_{1} a_{2} a_{3}} q_{2}^{\alpha^{\prime}}\left(-g^{\mu \tau}+\frac{q_{1}^{\mu} q_{1}^{\tau}}{m_{D^{*}}^{2}}\right) \\
\mathcal{M}_{\left[D^{*} D^{*} D\right]}= & G_{1} G_{2} G_{3} \varepsilon_{\alpha \beta \mu \nu} p_{i}^{\alpha} \varepsilon_{i}^{\beta} \varepsilon_{f \rho}^{*} p_{2 \sigma} \int \frac{d^{4} q_{2}}{(2 \pi)^{4}} \frac{\mathcal{F}}{a_{1} a_{2} a_{3}} \\
& \times\left(-g^{\mu \rho}+\frac{q_{1}^{\mu} q_{1}^{\rho}}{m_{D^{*}}^{2}}\right)\left(-g^{\nu \sigma}+\frac{q_{3}^{\nu} q_{3}^{\sigma}}{m_{D^{*}}^{2}}\right) .
\end{aligned}
$$




\section{References}

[1] D. M. Asner et al., Int. J. Mod. Phys. A 24, No. supp01 (2009) arXiv:0809.1869 [hep-ex]].

[2] W. Erni et al. [The PANDA Collaboration], M. F. Lutz, B. Pire and R. Timmermans, arXiv:0903.3905 [hep-ex].

[3] N. Brambilla et al. [Quarkonium Working Group], arXiv:hep-ph/0412158.

[4] N. Brambilla et al. [Quarkonium Working Group], arXiv:1010.5827 [hep-ph].

[5] E. S. Swanson, Phys. Rept. 429, 243 (2006) arXiv:hep-ph/0601110].

[6] E. Eichten, S. Godfrey, H. Mahlke and J. L. Rosner, Rev. Mod. Phys. 80, 1161 (2008) arXiv:hep-ph/0701208.

[7] M. B. Voloshin, Prog. Part. Nucl. Phys. 61, 455 (2008) [arXiv:0711.4556 [hep-ph]].

[8] S. Godfrey and S. L. Olsen, Ann. Rev. Nucl. Part. Sci. 58, 51 (2008) arXiv:0801.3867 [hep-ph]].

[9] N. Drenska, R. Faccini, F. Piccinini, A. Polosa, F. Renga and C. Sabelli, arXiv:1006.2741 [hep-ph].

[10] Y. J. Zhang, G. Li and Q. Zhao, Chin. Phys. C 34, 1181 (2010), Proceedings of the 5-th International Conference on Quarks and Nuclear Physics, 21-26 Sept. 2009, Beijing, China.

[11] X. Liu, B. Zhang and X. Q. Li, Phys. Lett. B 675, 441 (2009) arXiv:0902.0480 [hep-ph]].

[12] Y. J. Zhang, G. Li and Q. Zhao, Phys. Rev. Lett. 102, 172001 (2009) arXiv:0902.1300 [hep-ph]].

[13] X. Liu, X. Q. Zeng and X. Q. Li, Phys. Rev. D 74, 074003 (2006) arXiv:hep-ph/0606191.

[14] G. Li and Q. Zhao, Phys. Lett. B 670, 55 (2008) arXiv:0709.4639 [hep-ph]].

[15] C. Meng and K. T. Chao, Phys. Rev. D 77, 074003 (2008) [arXiv:0712.3595 [hep-ph]].

[16] C. Meng and K. T. Chao, Phys. Rev. D 78, 074001 (2008) arXiv:0806.3259 [hep-ph]].

[17] H. J. Lipkin, Nucl. Phys. B 291, 720 (1987).

[18] H. J. Lipkin and S. F. Tuan, Phys. Lett. B 206, 349 (1988).

[19] P. Moxhay, Phys. Rev. D 39, 3497 (1989).

[20] B. Borasoy, U.-G. Meißner and R. Nißler, Phys. Lett. B 643, 41 (2006) arXiv:hep-ph/0609010.

[21] G. Li, Y. J. Zhang and Q. Zhao, J. Phys. G 36, 085008 (2009) arXiv:0803.3412 [hep-ph]].

[22] G. Li, Q. Zhao and B. S. Zou, Phys. Rev. D 77, 014010 (2008) arXiv:0706.0384 [hep-ph]]. 
[23] J. J. Wu, Q. Zhao and B. S. Zou, Phys. Rev. D 75, 114012 (2007) arXiv:0704.3652 [hep-ph]].

[24] C. Hanhart, B. Kubis and J. R. Pelaez, Phys. Rev. D 76, 074028 (2007) arXiv:0707.0262 [hep-ph]].

[25] A. Faessler, T. Gutsche, V. E. Lyubovitskij and Y. L. Ma, Phys. Rev. D 76, 014005 (2007) arXiv:0705.0254 [hep-ph]].

[26] M. F. M. Lutz and M. Soyeur, Nucl. Phys. A 813, 14 (2008) arXiv:0710.1545 [hep-ph]].

[27] F.-K. Guo, C. Hanhart, S. Krewald and U.-G. Meißner, Phys. Lett. B 666, 251 (2008) arXiv:0806.3374 [hep-ph]].

[28] K. Gottfried, Phys. Rev. Lett. 40, 598 (1978).

[29] M. B. Voloshin, Nucl. Phys. B 154, 365 (1979).

[30] Y. P. Kuang, Front. Phys. China 1, 19 (2006). arXiv:hep-ph/0601044.

[31] H. Y. Zhou and Y. P. Kuang, Phys. Rev. D 44, 756 (1991).

[32] F.-K. Guo, C. Hanhart and U.-G. Meißner, Phys. Rev. Lett. 103, 082003 (2009) arXiv:0907.0521 [hep-ph]] [Erratum: ibid 104, 109901 (2010)].

[33] J. F. Donoghue and S. F. Tuan, Phys. Lett. B 164, 401 (1985).

[34] K. Maltman, Phys. Rev. D 44, 751 (1991).

[35] G. Ecker, J. Gasser, A. Pich and E. de Rafael, Nucl. Phys. B 321, 311 (1989).

[36] R. Urech, Nucl. Phys. B 433, 234 (1995) arXiv:hep-ph/9405341.

[37] B. L. Ioffe, Yad. Fiz. 29, 1611 (1979) [Sov. J. Nucl. Phys. 19, 827 (1979)].

[38] B. L. Ioffe and M. A. Shifman, Phys. Lett. B 95, 99 (1980).

[39] J. F. Donoghue and D. Wyler, Phys. Rev. D 45, 892 (1992).

[40] J. F. Donoghue, B. R. Holstein and D. Wyler, Phys. Rev. Lett. 69, 3444 (1992).

[41] H. Leutwyler, Phys. Lett. B 378, 313 (1996) arXiv:hep-ph/9602366.

[42] H. Mendez et al. [CLEO Collaboration], Phys. Rev. D 78, 011102 (2008) arXiv:0804.4432 [hep-ex]].

[43] J. Z. Bai et al. [BES Collaboration], Phys. Rev. D 70, 012006 (2004) arXiv:hep-ex/0403023.

[44] K. Nakamura et al. [Particle Data Group], J. Phys. G 37, 075021 (2010).

[45] S. Weinberg, in A Festschrift for I.I. Rabi, ed. by L. Motz, Trans. New York Acad. Sci. 38, 185 (1977).

[46] J. Gasser and H. Leutwyler, Phys. Rept. 87, 77 (1982). 
[47] H. Leutwyler, talk given in the Colloquium in memory of Jan Stern, 2-3 Oct. 2009, Paris, France.

[48] F.-K. Guo, C. Hanhart, G. Li, U.-G. Meißner and Q. Zhao, Phys. Rev. D 82, 034025 (2010) [arXiv:1002.2712 [hep-ph]].

[49] F.-K. Guo, C. Hanhart, U.-G. Meißner, Phys. Rev. Lett. 105, 162001 (2010). arXiv:1007.4682 [hep-ph]].

[50] B. Q. Li and K. T. Chao, Phys. Rev. D 79, 094004 (2009) [arXiv:0903.5506 [hep-ph]].

[51] M. Neubert, Phys. Rept. 245, 259 (1994) [arXiv:hep-ph/9306320].

[52] E. E. Jenkins, M. E. Luke, A. V. Manohar and M. J. Savage, Nucl. Phys. B 390, 463 (1993) arXiv:hep-ph/9204238.

[53] R. Casalbuoni, A. Deandrea, N. Di Bartolomeo, R. Gatto, F. Feruglio and G. Nardulli, Phys. Lett. B 302, 95 (1993).

[54] A. F. Falk, H. Georgi, B. Grinstein and M. B. Wise, Nucl. Phys. B 343, 1 (1990).

[55] J. D. Bjorken, talk given at Les Rencontre de la Valle d'Aoste, La Thuile, Italy, Mar 18-24, 1990, SLAC-PUB-5278.

[56] J. Hu and T. Mehen, Phys. Rev. D 73, 054003 (2006) arXiv:hep-ph/0511321.

[57] S. Fleming and T. Mehen, Phys. Rev. D 78, 094019 (2008) arXiv:0807.2674 [hep-ph]].

[58] R. Casalbuoni, A. Deandrea, N. Di Bartolomeo, R. Gatto, F. Feruglio and G. Nardulli, Phys. Lett. B 309, 163 (1993) arXiv:hep-ph/9304280.

[59] N. Brambilla, A. Pineda, J. Soto and A. Vairo, Rev. Mod. Phys. 77, 1423 (2005) arXiv:hep-ph/0410047].

[60] W. E. Caswell and G. P. Lepage, Phys. Lett. B 167, 437 (1986).

[61] E. Braaten, arXiv:hep-ph/9702225, talk given at the 3rd International Workshop on Particle Physics Phenomenology, Taipei, Nov. 1996.

[62] U.-G. Meißner and S. Steininger, Phys. Lett. B 419, 403 (1998) arXiv:hep-ph/9709453.

[63] E. E. Jenkins, Nucl. Phys. B 412, 181 (1994) arXiv:hep-ph/9212295.

[64] F.-K. Guo, C. Hanhart and U.-G. Meißner, JHEP 0809, 136 (2008) arXiv:0809.2359 [hep-ph]].

[65] R. F. Dashen, Phys. Rev. 183, 1245 (1969).

[66] G. Burdman and J. F. Donoghue, Phys. Lett. B 280, 287 (1992).

[67] M. B. Wise, Phys. Rev. D 45, R2188 (1992).

[68] T. M. Yan, H. Y. Cheng, C. Y. Cheung, G. L. Lin, Y. C. Lin and H. L. Yu, Phys. Rev. D 46, 1148 (1992) [Erratum-ibid. D 55, 5851 (1997)]. 
[69] R. Casalbuoni, A. Deandrea, N. Di Bartolomeo, R. Gatto, F. Feruglio and G. Nardulli, Phys. Rept. 281, 145 (1997) arXiv:hep-ph/9605342.

[70] P. Colangelo, F. De Fazio and T. N. Pham, Phys. Rev. D 69, 054023 (2004) arXiv:hep-ph/0310084].

[71] F.-K. Guo, C. Hanhart and U.-G. Meißner, Phys. Rev. Lett. 102, 242004 (2009) arXiv:0904.3338 [hep-ph]].

[72] P. Colangelo, F. De Fazio and T. N. Pham, Phys. Lett. B 542, 71 (2002) arXiv:hep-ph/0207061.

[73] S. Uehara et al. [Belle Collaboration], Phys. Rev. Lett. 96, 082003 (2006) arXiv:hep-ex/0512035].

[74] E. J. Eichten, K. Lane and C. Quigg, Phys. Rev. D 73, 014014 (2006) [Erratum-ibid. D 73, 079903 (2006)] arXiv:hep-ph/0511179].

[75] S. Uehara et al. [Belle Collaboration], Phys. Rev. Lett. 104, 092001 (2010) arXiv:0912.4451 [hep-ex]].

[76] X. Liu, Z. G. Luo and Z. F. Sun, Phys. Rev. Lett. 104, 122001 (2010) arXiv:0911.3694 [hep-ph]].

[77] T. Barnes, S. Godfrey and E. S. Swanson, Phys. Rev. D 72, 054026 (2005) arXiv:hep-ph/0505002.

[78] S. K. Choi et al. [Belle Collaboration], Phys. Rev. Lett. 91, 262001 (2003) arXiv:hep-ex/0309032.

[79] N. A. Törnqvist, Phys. Lett. B 590, 209 (2004) arXiv:hep-ph/0402237.

[80] E. Braaten and M. Lu, Phys. Rev. D 76, 094028 (2007) arXiv:0709.2697 [hep-ph]].

[81] C. Hanhart, Yu. S. Kalashnikova, A. E. Kudryavtsev and A. V. Nefediev, Phys. Rev. D 76, 034007 (2007) arXiv:0704.0605 [hep-ph]].

[82] Yu. S. Kalashnikova and A. V. Nefediev, Phys. Rev. D 80, 074004 (2009) arXiv:0907.4901 [hep-ph]].

[83] C. Bignamini, B. Grinstein, F. Piccinini, A. D. Polosa and C. Sabelli, Phys. Rev. Lett. 103, 162001 (2009) [arXiv:0906.0882 [hep-ph]].

[84] P. Artoisenet and E. Braaten, arXiv:0911.2016 [hep-ph].

[85] S. G. Matinyan and B. Müller, Phys. Rev. C 58, 2994 (1998) arXiv:nucl-th/9806027.

[86] A. Deandrea, G. Nardulli and A. D. Polosa, Phys. Rev. D 68, 034002 (2003) arXiv:hep-ph/0302273.

[87] R. D. Matheus, F. S. Navarra, M. Nielsen and R. Rodrigues da Silva, Phys. Lett. B 541, 265 (2002) arXiv:hep-ph/0206198.

[88] K. L. Haglin and C. Gale, Phys. Rev. C 63, 065201 (2001) arXiv:nucl-th/0010017. 
[89] M. Ablikim et al. [The BESIII Collaboration], Phys. Rev. Lett. 104, 132002 (2010) arXiv:1002.0501 [hep-ex]].

[90] J. J. Wu and B. S. Zou, Phys. Rev. D 78, 074017 (2008) arXiv:0808.2683 [hep-ph]].

[91] N. N. Achasov, S. A. Devyanin and G. N. Shestakov, Phys. Lett. B 88, 367 (1979).

[92] S. Dubynskiy and M. B. Voloshin, Phys. Rev. D 77, 014013 (2008) arXiv:0709.4474 [hep-ph]].

[93] H. Y. Cheng, C. K. Chua and A. Soni, Phys. Rev. D 71, 014030 (2005) arXiv:hep-ph/0409317]. 